submitted to Geophys. J. Int.

\title{
Thermal Nature and Resolution of the
}

\section{Lithosphere-Asthenosphere Boundary Under the Pacific From Surface Waves}

\author{
C. Beghein ${ }^{1}$, Z. Xing ${ }^{1}$ and S. Goes ${ }^{2}$ \\ ${ }^{1}$ Department of Earth, Planetary, and Space Sciences, University of California Los Angeles, Los Angeles, \\ CA 90095, USA, \\ ${ }^{2}$ Faculty of Engineering, Department of Earth Science and Engineering, Imperial College, London, UK
}

\section{SUMMARY}

It is strongly debated whether the interface between the lithosphere and underlying asthenosphere is a temperature-dependent rheological transition, as expected in a thermal convection system, or additionally affected by the presence of melts and/or fluids. Previous surface wave studies of Pacific oceanic lithosphere have found that shear velocity and azimuthal anisotropy vary with seafloor crustal age as expected for a thermal control; however radial anisotropy does not. Various thermo-mechanical models have been proposed to explain this disparate behaviour. Nonetheless, it is unclear how robust the surface wave constraints are, and this is what we test in this study. We apply a Bayesian model space search approach to three published Pacific surface-wave dispersion datasets, two phase-velocity and one combined phase- and group-velocity set, and determine various proxies for the depth of the lithosphere-asthenosphere boundary (LAB) and their uncertainties based on the velocity and radial anisotropy model distributions obtained. In their overall character and pattern with age, the velocity models from different datasets are consistent with each other, although they differ in their values of LAB depths. Uncertain- 


\section{Beghein, C., Xing, Z. and Goes, S.}

ties are substantial (as much as $20 \mathrm{~km}$ on LAB depths) and the addition of group-velocity data does not reduce them. Radial anisotropy structures differ even in pattern and display no obvious age dependence. However, given the uncertainties, we cannot exclude that radial anisotropy, azimuthal anisotropy, and velocity models actually reflect compatible, age-dependent, LAB depth estimates. The velocity LAB trends are most like those expected for half-space cooling, because velocity differences persist at old ages, below the depth of common plate cooling models. Any direct signature of sub-ridge melt would be too small-scale to be resolved by these data. However, the velocity-increasing effects of dehydration and depletion due to melting below the ridge could explain why LAB proxy depths tend to a minimum of $\sim 60 \mathrm{~km}$ below young ocean floor.

Key words: Seismic anisotropy - Tomography - Inverse theory - Probability distributions - Statistical seismology - Surface waves and free oscillations.

\section{INTRODUCTION}

The transition from the lithosphere, the mechanically strong plate at the surface, to the deeper and more ductile asthenosphere, is key to better understand plate tectonics on Earth and our planet's thermochemical evolution. However, the nature of this transition, often called the LAB (lithosphereasthenosphere boundary), remains elusive as several factors such as temperature, chemical composition, partial melt, water content, and grain size may affect the magnitude and sharpness of this rheological contrast (see review by Fischer et al. (2010)). Constraining the depth of the LAB and how it varies between tectonic blocks or with ocean crustal age can help determine the relative importance of these factors.

A variety of geological, geochemical and geophysical observables can be used to obtain information about the LAB (Eaton et al., 2009) and to determine its depth. However, different proxies can lead to different conclusions. For instance, sea-floor magnetotelluric studies reveal the presence of a highconductivity layer beneath the lithosphere (Filloux, 1980; Chave et al., 1981; Lizarralde et al., 1995; Naif et al., 2013). Several authors have favored the presence of partial melt to explain these observations (Shankland et al., 1981; Naif et al., 2013), although some have suggested the presence of water is responsible (Lizarralde et al., 1995). Based on their measurements, Naif et al. (2013) additionally proposed that the LAB under young oceanic plates consists of a thin, partially molten, low-viscosity 
layer located between 45 and $70 \mathrm{~km}$, and acts as a lubricant, enhancing decoupling of the overlying lithosphere from the convecting mantle.

Seismological studies can also be used to constrain the depth of the LAB, but results and their interpretation vary as well. The top of the asthenosphere is often associated with a sharp decrease of several percent in wave velocity. It is called the Gutenberg discontinuity in oceanic settings where it has been observed with SS precursor data, and with $\mathrm{ScS}$ and sScS reflected waves (Revenaugh and Jordan, 1991; Gaherty et al., 1996; Bagley and Revenaugh, 2008; Rychert and Shearer 2011; Schmerr, 2012; Tharimena et al., 2017a). It can also be observed with receiver functions, but their usage is mostly limited to continents, ocean islands, and ocean bottom seismometers because it can only map reflectors located underneath a seismic station (Kawakatsu et al., 2009; Courtier and Leahy, 2011). The sharpness and lack of clear crustal age dependence of this discontinuity, at least for ages $>36 \mathrm{Myr}$ (Tharimena et al., 2017a), call for a compositional origin of the observed discontinuities. Partial melt as well as the effect of dehydration at the ridge have been invoked to explain these observations (Anderson and Sammis, 1970; Gaherty et al., 1996; Hirth and Kohlstedt, 1996; Karato and Jung, 1998; Kawakatsu et al., 2009; Karato, 2012; Schmerr, 2012; Sakamaki et al., 2013; Olugboji et al., 2016; Tharimena et al., 2017a). Melt is also often invoked to explain the origin of the discontinuity in continents (Tharimena et al., 2017b).

By contrast, surface wave tomography has yielded shear-wave velocity and azimuthal anisotropy models under oceans that display a dependence on seafloor age, suggesting that oceanic plate formation is primarily controlled by temperature (Nishimura and Forsyth, 1989, Ritzwoller et al, 2004; Maggi et al., 2006; Nettles and Dziewoński, 2008; Debayle and Ricard, 2013; Becker et al., 2014; Beghein et al., 2014; Burgos et al., 2014; Schaeffer et al., 2016; Ma and Dalton, 2017). These models resolve a relatively high velocity layer atop a low velocity zone (Nishimura and Forsyth, 1989; Maggi) et al., 2006; Nettles and Dziewoński, 2008; Debayle and Ricard, 2013; Becker et al., 2014; Beghein et al., 2014; Burgos et al., 2014; Schaeffer et al., 2016). In many cases, the thickness of the top layer increases with the age of the ocean crust, following to first order a half space cooling (HSC) model, in which thickness increases with the square root of age (Parsons and Sclater, 1977). In a few cases, a plate cooling model, where the thickness at older ages is limited to a maximum of around $100 \mathrm{~km}$ by either small-scale instabilities or reheating (Stein and Stein, 1992) was found to be a better fit to both the seismic data and seafloor topography (Ritzwoller et al, 2004; Ma and Dalton, 2017). Azimuthal anisotropy models derived from surface waves are often characterized by two layers, with fast axes that follow ocean ridge paleospreading directions in the top layer and parallel to the geodetically determined present-day direction of plate motion (Gripp and Gordon, 1990; Gripp and Gordon, 1992) in the lower layer (Smith et al., 2004; Maggi et al., 2006; Debayle and Ricard, 2013; Becker et al., 2014; 


\section{Beghein, C., Xing, Z. and Goes, S.}

Beghein et al., 2014; Burgos et al., 2014; Schaeffer et al., 2016). In addition, the depth at which the azimuthal anisotropy is well aligned with the plate motion direction increases with plate age and tends to display a HSC age-dependence (e.g., Debayle and Ricard (2013) or Beghein et al. (2014)).

Interestingly, however, three radial anisotropy models published in the last decade and resulting from regularized inversions of surface wave data do not display the same age dependence as their isotropic or azimuthally anisotropic counterparts (Nettles and Dziewoński, 2008; Beghein et al., 2014; Burgos et al., 2014). Instead, they tend to be characterized by an upper layer where $V_{S V}>V_{S H}$ and a lower layer where $V_{S V}<V_{S H}$, with a transition that occurs at the same depth of $\sim 60-80 \mathrm{~km}$ for all ages, i.e. it does not show any significant age dependence. Lattice-preferred orientation (LPO) of olivine is thought to be the primary contribution to the seismic anisotropy observed in the upper mantle. Laboratory experiments show that, in most cases, when an aggregate of olivine crystals is deformed by simple shear in a dislocation creep regime, the fast seismic direction aligns with the maximum shear direction, which is also the flow direction in the simple case of horizontal flow with a vertical velocity gradient (Zhang and Karato, 1995; Karato et al., 2008). The anisotropy in the lower layer of the radial anisotropy models cited above can thus be interpreted as the signature of horizontal mantle flow in the asthenosphere, and the upper layer could be associated with the lithosphere. If the depth of the change in radial anisotropy is used as a proxy for the $\mathrm{LAB}$, then radial anisotropy predicts an age-independent flat oceanic LAB, which cannot be due to thermal effects and appears to contradict results based on azimuthal anisotropy or three-dimensional isotropic velocity models.

Below the Pacific Ocean, Beghein et al. (2014) found that the relatively constant depth of the Gutenberg discontinuity coincides with high vertical gradients in azimuthal anisotropy and is located within a seismic lithosphere that thickens with age. They suggested that the Gutenberg discontinuity results from dehydration of the mantle underlying the mid-ocean ridge, resulting in a chemically depleted, viscous layer that translates away from the spreading center and becomes overprinted by lower temperatures as the plate cools down. Similarly, the quasi age-independent change in radial anisotropy at about $70 \mathrm{~km}$ depth could reflect different processes in the formation and evolution of oceanic plates than those seen by azimuthal anisotropy and isotropic velocities. Hier-Majumder and Drombosky (2015) explained the observed transition in radial anisotropy with calculations of melt redistribution at the base of the lithosphere. However, this does not explain the presence of azimuthal anisotropy unless the melt layers are significantly inclined from horizontal. Interestingly, Hedjazian et al. (2017) were able to reproduce a radial anisotropy pattern in agreement with the tomographic models without introducing any partial melt or dehydration. Using simple 2-D surface-driven mid-ocean ridge flow calculations with slow dynamic recrystallization and a mix of diffusion and dislocation creep rheology, they showed that LPO evolution results in radial anisotropy with a maximum posi- 
tive gradient at a constant depth. The observed age-independent radial anisotropy signal could thus reflect the natural strain history of olivine crystals, even if the azimuthal anisotropy and velocity proxies for the LAB are thermally controllled. Similarly, with a combination of laboratory-based torsion experiments on olivine aggregates and flow modelling for a viscous fluid deforming in simple shear, Hansen et al. (2016) were able to predict both radial and azimuthal anisotropy patterns in agreement with the tomographic models. They obtained an age-dependent azimuthal anisotropy, consistent with thermal effects, and no age signal in the depth of the radial anisotropy change. Neither of these two last studies precludes the effect of dehydration or the presence of melt, but as pointed out by Hansen et al. (2016) the melt fraction should be small $(<1 \%)$ to not affect the developed anisotropy. Several researchers have additionally shown that the asthenospheric low seismic velocities found under oceans and continents can be explained by purely thermal effects without any melt requirement simply by accounting for the effect of anelasticity on wavespeed (Karato and Jung, 1998; Röhm et al., 2000; Faul and Jackson, 2005; Stixrude and Lithgow-Bertelloni, 2005; Goes et al., 2012).

Clearly, the apparent discrepancies between radial and azimuthal anisotropy models and their implications for our understanding of the origin of the LAB has gained recent attention. However, while the two types of anisotropy may be reconciled with simple flow models provided well-chosen parameters, the anisotropy models derived from regularized inversions of surface wave dispersion data are inherently non-unique, leading to the question whether the lack of age dependence in the vertical change of radial anisotropy is sufficiently resolved. Since the interpretation of tomographic models is only meaningful if the interpreted features are well resolved, it is important to determine how well constrained the shear-wave velocity $\left(V_{S}\right)$ and radial anisotropy $(\xi)$ models are.

A previous study (Bartzsch et al., 2011) investigated how sensitive Rayleigh wave phase velocity dispersion curves are to the depth and thickness of the LAB, parameterized as a linear transition from a high-velocity lithospheric layer to a low-velocity asthenosphere. Their synthetic tests and real data applications to continental regions show that Rayleigh wave phase velocities between $22 \mathrm{~s}$ and $200 \mathrm{~s}$ have little resolution for LAB thickness, in agreement with previous work by Eaton et al. (2009), but can constrain transition depth quite well. However, no quantitative uncertainties were determined for any of these two variables, and the criteria to determine whether the LAB depth is well-constrained was based on an arbitrary misfit cutoff. In addition, the authors did not investigate the sensitivity of surface wave data to the LAB depth when both Love and Rayleigh waves are inverted jointly for $V_{S}$ and $\xi$, neither did they test other LAB depth proxies such as the change in radial anisotropy with depth.

The resolution tests performed by Beghein et al. (2014) using the fundamental mode Love and Rayleigh wave phase velocity data of Visser et al. (2008a) showed that the vertical resolution of their radial anisotropy model of the Pacific upper mantle was poor. In this paper, we further explore the 


\section{Beghein, C., Xing, Z. and Goes, S.}

resolution of radial anisotropy and shear wavespeed in the Pacific upper mantle using a Bayesian model space technique to determine whether the age-independence of the vertical change in radial anisotropy is resolved by surface wave data. This approach enables us to explore the model space, including the null-space, and obtain quantitative uncertainties on $V_{S}$ and $\xi$. We can define a LAB depth proxy based on $V_{S}$ and one based on $\xi$, and from the distribution of acceptable $V_{S}$ and $\xi$ models, we can determine the distribution of possible LAB depths for each proxy. This allows us to test whether, among all the good data-fitting radial anisotropy models, there are any displaying an age dependence similar to that seen in the velocity models or whether $V_{S}$ and $\xi$ reflect different processes.

Finally, we tested the hypothesis that asthenospheric low seismic velocities may have a solid state origin and can be explained purely by thermal effects, including those on seismic attenuation, without any requirement for an additional effect of melt as advocated by several authors (Karato and Jung, 1998; Röhm et al., 2000; Faul and Jackson, 2005; Stixrude and Lithgow-Bertelloni, 2005; Goes et al., 2012). The contribution of melt to the seismic properties of the asthenosphere has long been a matter of debate with some researchers arguing for significant melt fractions near ridges to explain low S-wave velocities (Forsyth et al., 1998; Harmon et al., 2009) and to explain the Gutenberg discontinuity, as discussed above (Anderson and Sammis, 1970; Kawakatsu et al., 2009; Tharimena et al., 2017b), and others favoring low amounts of melt based on petrological studies (Asimow et al., 2004; Hirschmann, 2010) or the efficiency of melt transport inferred from the decay of $\mathrm{Ra}, \mathrm{Pa}$ and Th (Lundstrom, 2003; Rubin et al., 2005; Stracke et al., 2006). Here we tested our models against synthetic models of seismic velocities including anelastic effects computed as in Goes et al. (2012) for dry and wet models. We additionally tested whether our models can discriminate between half space (HS) and plate (PL) cooling models.

\section{DATA}

\subsection{Surface Waves}

At any given point at the surface of the Earth, the measured phase and group velocity perturbation at frequency $\omega$ is the vertical average of the underlying elastic structure weighted by sensitivity kernels (Woodhouse and Dahlen, 1978; Montagner and Nataf, 1986):

$$
\alpha_{0}(\omega)=\int_{0}^{a} \mathbf{K}_{m}(r, \omega) d \ln \mathbf{m}(r) d r
$$

$\ln \mathbf{m}(r)=d \mathbf{m}(r) / \mathbf{m}_{\mathbf{0}}(r)$ represents relative perturbations in parameter $\mathbf{m}$ with respect to a reference model $\mathbf{m}_{\mathbf{0}}$, and $a$ stands the radius of the Earth. $K_{m}(r)$ are the partial derivatives or sensitivity kernels for model parameter $\mathbf{m}$. They can be calculated for any 1-D Earth model using normal mode theory (Takeuchi and Saito, 1972; Rodi et al., 1975) and vary from mode to mode. 
In this study, we tested three surface wave velocity datasets from two different groups to assess the variability of the results with the dataset employed. The first dataset, hereafter labeled dataset 1 , consisted of the isotropic part of the fundamental mode Rayleigh and Love wave global phase velocity maps obtained by Visser et al. (2008a), which were used by Beghein et al. (2014). They were obtained for 16 periods between $35 \mathrm{~s}$ and $175 \mathrm{~s}$ and resulted from the inversion of over 60, 000 fundamental mode ( $n=0$ ) Rayleigh wave dispersion curves and over 45, 000 dispersion curves for Love waves. Visser et al. (2008a) determined that, on average, the isotropic term of the fundamental mode phase velocity maps have a resolution comparable to that of a spherical harmonic expansion of degree order 25 , yielding a lateral resolution of about $1600 \mathrm{~km}$ in the uppermost mantle. The second dataset (dataset 2) consisted of the Ekström (2011) Love and Rayleigh wave global phase velocity maps for 15 different periods between $25 \mathrm{~s}$ and $250 \mathrm{~s}$. These maps were obtained by inversion of between 18, 000 and 85, 000 Rayleigh wave dispersion curves and betweend 78,000 and 286,000 Love wave dispersion curves, depending on the period. Ekström (2011) estimated that a nominal lateral resolution of $650 \mathrm{~km}$ was achieved in most areas. Examples of phase velocity maps for both dataset 1 and dataset 2 at a period of $35 \mathrm{~s}$ and $100 \mathrm{~s}$ are shown in Fig. 1. Each dataset was corrected for the effects of 3-D structure in the crust using non-linear corrections as described in section 2.2 .

Other authors have argued that crustal corrections may not be appropriate for Rayleigh wave phase velocities at periods $<60 s$ (Bozdag and Trampert, 2008). Even though this issue is still being debated (Bozdag and Trampert, 2008, Parisi and Ferreira, 2016), we decided to account for this possibility and constructed a third dataset composed of the Ekström (2011) phase velocity maps at periods $>60 s$ and the 25-250 s global group velocity maps obtained by the same author. The group velocity data were added to compensate the loss of sensitivity in the shallow mantle resulting from the removal of the short period phase velocity dispersion data. Fig. 2 shows examples of those group velocity maps at $25 \mathrm{~s}$ and $75 \mathrm{~s}$, and Fig. 3 compares sensitivity kernels for each dataset. This illustrates how dataset 2, with its broader frequency range, is more sensitive to structure in the uppermost $50 \mathrm{~km}$ than dataset 1 , which could yield better vertical resolution. The partial derivatives of dataset 3 , which is composed of the mid-to-high period phase velocity data of dataset 2 and of group velocities, have larger amplitudes than the other two datasets, especially in the upper $50 \mathrm{~km}$ where group velocities have greater sensivity to structure than the selected phase velocities.

\subsection{Crustal Corrections}

Because surface wave velocities are highly sensitive to crustal structure and crustal thickness, it is important to account for the effect of the crust on the measurements to minimize the mapping of the crust into the mantle model (Boschi and Ekström, 2002, Panning et al., 2010). This is especially 


\section{Beghein, C., Xing, Z. and Goes, S.}

important when modelling radial anisotropy because Love waves are sensitive to shallower structure than Rayleigh waves at the same period. Incorrectly accounting for lateral variations in crustal structure could thus affect the anisotropic mantle model (Ferreira et al., 2010). For waveform inversions, a treatment of the crust proposed by Lekić and Romanowicz (2011) consists of constructing a prior crustal model that fits global fundamental mode surface wave dispersion measurements, and using this model in the computation of predicted waveforms. Other techniques involve correcting dispersion data with predictions from a prior crustal model before inverting those data (Boschi and Ekström, 2002; Kustowski et al., 2007; Marone and Romanowicz, 2007; Ferreira et al., 2010; Panning et al., 2010) or inverting crustal thickness and/or structure jointly with mantle structure (Visser et al., 2008b). Alternatively, a hybrid two-step method can be employed (Burgos et al., 2014; Chang et al., 2014).

In this work we used crustal model CRUST1.0 (Laske et al., 2013) to calculate non-linear crustal corrections. CRUST1.0 is a $1^{\circ} \times 1^{\circ}$ model in which the crust is represented as a stack of layers (ice or water, sediments and crystalline crust) with assigned thicknesses as well as P- and S-wave velocities and density. It was built using Earth's global relief model ETOPO1 (Amante and Eakins, 2009) for seafloor bathymetry, ice thickness, and surface topography, and active source seismic data, receiver functions, and group velocity data to constrain the Moho depth and crustal velocities. Xing and Beghein (2015) showed that, for this type of correction, the choice of the crustal model does not significantly affect the resulting upper mantle velocity or radial anisotropy model, especially in oceanic areas. They demonstrated that differences between oceanic $V_{S}$ and $\xi$ models obtained after correcting phase velocity data with different crustal models are smaller than the intrinsic uncertainties of the models due to the non-unique nature of the inverse problem.

We calculated non-linear crustal corrections following a procedure similar to that of Boschi and Ekström (2002). We first constructed a 3-D reference model composed of the PREM (Dziewonski and Anderson, 1981) mantle and CRUST1.0 for the crust. We divided Earth's surface into cells and used the local 1-D model at each grid cell $j$ with computer program MINEOS to calculate local, theoretical phase $c_{j}$ and group velocities $U_{j}$ and local eigenfunctions, which were then used to determine local sensitivity kernels $K_{m, j}$, where $m$ represents a model parameter. At each grid cell $j$, the difference between the phase $c_{j}$ or group velocity $U_{j}$ predicted by the 3-D model and that calculated from PREM ( $c_{0}$ or $U_{0}$ ) is the contribution of the crustal model to the measured velocity perturbation $d c_{c, j}$ or $d U_{c, j}$, i.e., it is the crustal correction. After removing this correction from the measured phase or group velocity anomaly, we are left with a corrected signal $\left(\frac{d c_{c}}{c}\right)_{j}$ or $\left(\frac{d U_{c}}{c}\right)_{j}$ that represents the phase or group velocity anomaly due to a local mantle perturbation with respect to the reference model at grid cell $j$. This remaining signal can thus be inverted to infer local perturbations in mantle structure with respect to PREM using the local sensitivity kernels: 


$$
\left(\frac{d c_{c}}{c}\right)_{j}=\int_{0}^{M o h o} \sum_{i}\left[K_{m_{i}, j}(r) d \ln m_{i, j}(r)\right] d r
$$

where the sum over $i$ is carried out over all model parameters. A similar equation applies to group velocities. Note that that this method is only strictly valid if there is no mode coupling since it assumes that the effect of crustal structure can be computed independently at each location on the Earth's surface. Examples of the corrected phase and group velocity maps are shown in Fig. 4 and comparison with Figs. 1 and 2 demonstrates that the crust contributes significantly to the measurements.

\section{METHOD}

\subsection{Forward Problem and Parameterization}

As shown by Eq. 1, the measured phase and group velocity perturbations are weighted depth averages of the underlying structure, and the dispersive properties of surface waves imply that the longer period data are sensitive to deeper structure than the short period waves (Fig. 3). We note that, as opposed to mode-based waveform inversion techniques, an implicit assumption in Eq. 1 1 is that there is no mode coupling. This is clearly an approximation since we know that lateral heterogeneities and anisotropy can cause coupling between modes (e.g., Park and Yu (1992), Beghein et al. (, 2008)).

In an isotropic medium, the model parameters are density $\rho$ and two elastic coefficients: the Lamé parameter $\lambda$ and the shear modulus $\mu$, or alternatively the bulk modulus $\kappa$ and the shear modulus. Eq. 1 becomes:

$$
\alpha_{0}(\omega)=\int_{0}^{a}\left[K_{\rho}(r, \omega) d \ln \rho(r)+K_{\mu}(r, \omega) d \ln \mu(r)+K_{\kappa}(r, \omega) d \ln \kappa(r)\right] d r
$$

In a general anisotropic medium, a total number of 21 independent parameters is needed to fully describe the 4th order elastic stiffness tensor. Because seismic data cannot resolve all 21 parameters, we make assumptions regarding the symmetry of the anisotropic medium to reduce the number of unknowns. In the simple case of radial anisotropy, the medium has hexagonal symmetry with a vertical symmetry axis and can be described by five independent parameters: the velocity of vertically polarized shear waves $V_{S V}$, of horizontally polarized shear waves $V_{S H}$, of vertically and horizontally propagating P-waves $V_{P V}$ and $V_{P H}$, respectively, and parameter $\eta$ which describes wave propagation at an intermediate angle:

$$
\begin{aligned}
\alpha_{0}(\omega)=\int_{0}^{a} \quad\left[K_{V_{P V}}(r, \omega) d \ln V_{P V}(r)+K_{V_{P H}}(r, \omega) d \ln V_{P H}(r)+K_{V_{S V}}(r, \omega) d \ln V_{S V}(r)\right. \\
\\
\left.+K_{V_{S H}}(r, \omega) d \ln V_{S H}(r)+K_{\eta}(r, \omega) d \ln \eta(r)+K_{\rho}(r, \omega) d \ln \rho(r)\right] d r
\end{aligned}
$$

A parameterization in terms of the Love elastic parameters $A, C, N, L$, and $F$ (Love, 1927) is sometimes employed (Beghein, 2010; Chang et al., 2014), with $A=\rho V_{P H}^{2}, C=\rho V_{P V}^{2}, N=\rho V_{S H}^{2}$, 
10 Beghein, C., Xing, Z. and Goes, S.

$L=\rho V_{S V}^{2}, F=(A-2 L) \eta$. Here, we chose instead to follow Panning and Romanowicz (2006) and used another equivalent parameterization:

$$
\begin{aligned}
V_{P}^{2} & =\left(V_{P V}^{2}+4 V_{P H}^{2}\right) / 5 \\
V_{S}^{2} & =\left(2 V_{S V}^{2}+V_{S H}^{2}\right) / 3 \\
\phi & =V_{P V}^{2} / V_{P H}^{2} \\
\xi & =V_{S H}^{2} / V_{S V}^{2} \\
\eta & =F /(A-2 L)
\end{aligned}
$$

where $V_{P}$ and $V_{S}$ are Voigt average isotropic velocities representing upper bounds on the effective elastic moduli (Babuška and Cara, 1991). $\phi$ and $\xi$ represent P-wave and S-wave radial anisotropy, respectively. This parameterization enables us to directly invert for anisotropy instead of calculating a posteriori from separate inversions of $V_{S V}$ and $V_{S H}$, which can cause unwanted roughness in the mapped anisotropic structure (Nettles and Dziewoński, 2008). Eq. 1 therefore becomes:

$$
\begin{aligned}
{ }_{k} \alpha_{0}=\int_{0}^{a} & {\left[\quad{ }_{k} K_{V_{P}}(r) d \ln V_{P}(r)+{ }_{k} K_{V_{S}}(r) d \ln V_{S}(r)+{ }_{k} K_{\phi}(r) d \ln \phi(r)\right.} \\
& \left.+{ }_{k} K_{\xi}(r) d \ln \xi(r)+{ }_{k} K_{\eta}(r) d \ln \eta(r)+{ }_{k} K_{\rho}(r) d \ln \rho(r)\right] d r
\end{aligned}
$$

where $k$ represents the mode considered and $\omega$ is omitted for clarity. Not all of these six parameters are, however, well resolved by surface waves because of reduced sensitivity to $V_{P}, \phi, \eta$ and $\rho$ and because of parameter trade-offs. For this reason, we used the empirical relationships established by Montagner and Anderson (1989) from petrological considerations to scale perturbations in compressional wave velocity and density to changes in shear wave velocity, and to scale changes in compressional wave anisotropy and in $\eta$ to perturbations in shear wave anisotropy:

$$
\begin{aligned}
\frac{d \ln V_{P}}{d \ln V_{S}} & =0.5 \\
\frac{d \ln \rho}{d \ln V_{S}} & =0.33 \\
\frac{d \ln \phi}{d \ln \xi} & =-2.5 \\
\frac{d \ln \eta}{d \ln \xi} & =-1.5
\end{aligned}
$$

We parameterized the Earth horizontally by dividing its surface into $5^{\circ} \times 5^{\circ}$ cells, and conducted the inversions of eq. 10 for each cell separately. At every grid cell, we used a depth parameterization for $\operatorname{d} \ln V_{S}(r)$ and $\operatorname{dln} \xi(r)$ composed of $M=8$ cubic spline function $S_{i}(r)$ (i=1,..,M) for datasets 1 and 2, and $M=9$ splines for dataset 3 because of the increased sensitivity at shallow depths (Fig. 5):

$$
d \ln V_{S}(r)=\sum_{i=1}^{M} d \ln V_{S}^{i} S_{i}(r)
$$




$$
d \ln \xi(r)=\sum_{i=1}^{M} d \ln \xi^{i} S_{i}(r)
$$

where $\operatorname{dn} V_{S}^{i}$ and $\operatorname{dln} \xi^{i}$ are the coefficients of the ith spline. Note that, since the top of the splines is defined by the local Moho and not Earth's surface, the spline functions are varying laterally. The vertical spacing between the splines is also variable to reflect changes in the depth sensitivity of the data. Eq. 10 can be written as:

$$
\mathbf{G m}=\mathbf{d}
$$

where vector $\mathbf{d}$ represents the data vector, and $\mathbf{m}$ is the vector containing the model parameters, i.e. the $\operatorname{dnn} V_{S}^{i}$ and $\operatorname{dln} \xi^{i}$ of eqs. 15 and 16 . Matrix $\mathbf{G}$ is the kernel matrix and its elements $G_{k i}$ can be calculated using the prior constraints of eqs. 11 |14 and by integrating the sensitivity kernels projected onto the spline functions $S_{i}(r)$ :

$$
G_{k i}=\int_{0}^{a}\left[{ }_{k} K_{V_{S}}(r)+0.5_{k} K_{V_{P}}(r)+0.33_{k} K_{\rho}(r)+{ }_{k} K_{\xi}(r)-2.5_{k} K_{\phi}(r)-1.5_{k} K_{\eta}(r)\right] S_{i}(r) d r
$$

At each grid cell, we have 16 model parameters and 16 Rayleigh and 16 Love wave phase velocities for dataset 1. Dataset 2 contains 15 Rayleigh and 15 Love wave phase velocities. Dataset 3 contains 7 Rayleigh and 5 Love wave phase velocities, as well as 15 Rayleigh and 15 Love group velocities. Matrix $\mathbf{G}$ has thus 32 rows and 16 columns for dataset 1, 30 rows and 16 columns for dataset 2, and 42 rows and 18 columns for dataset 3 .

\subsection{Modelling}

Regularized inversions do not always provide reliable quantitative model uncertainties, especially in the presence of a large null space (Trampert, 1998). An alternative approach can be found in direct search techniques. Without needing to implicitly introduce strong a priori information, this type of method is able to explore the model space, including null-space, giving us a better description of the range of possible models instead of just one "best". Here we utilized a two-part global optimization technique, namely the Neighborhood Algorithm (NA) (Sambridge, 1999a; Sambridge, 1999b).

The first part of NA is a direct search of the model space. First, a number of samples are randomly generated in the model space and the model space is divided into Voronoi cells using this initial sampling. Each cell is centered on one of the models by construction. An approximate misfit surface is determined by calculating the misfit of each of these models. At each subsequent iteration, $n_{s}$ new samples are then generated by performing a uniform random walk with a Gibbs sampler in the Voronoi cell of each of the $n_{r}$ best fitting models. The Voronoi cells are updated to accommodate the newly generated models, and misfits are calculated for those new models, determining a new misfit surface. Misfits are then ranked among all the existing models to determine the next set of $n_{r}$ best 


\section{Beghein, C., Xing, Z. and Goes, S.}

fitted models. At each iteration, the sampling density therefore increases in the neighborhoods of the better data-fitting models, and the NA is able to use the information contained in the previous models to adapt the sampling. The choice of $n_{r}$ and $n_{s}$ is generally decided by trial and error to control the convergence speed and the sampling quality. A small $n_{s} / n_{r}$ ratio implies a slower convergence of the algorithm but helps perform a sampling of the model space as thorough as possible to avoid getting trapped in a local minimum. The iteration is stopped by the user when the misfit does not show any clear decrease with each iteration.

In this work, we first carried out regularized inversions of the surface wave data using eq. 17 and the singular value decomposition (SVD) method of Matsu'ura and Hirata (1982). This technique is described in details in Yuan and Beghein (2014). We then ran the first part of the NA using a uniform prior model distribution around the results of the regularized inversion: each of the 16 model parameters were allowed to vary by $5 \%$ with respect to the local reference model and around the inversion results. Searching the model space around the inversion results has the advantage of accelerating the sampling convergence if the model distribution is close to a Gaussian while still allowing the NA to find good data fitting models away from the inversion results. We compared our results with those resulting from a model space search around zero and showed they are equivalent (see section 4).

In the second part of the NA, a Bayesian appraisal of the ensemble of models obtained from sampling the model space is performed. Unlike other statistical techniques, such as importance sampling, that draw inferences on the models using only a subset of the ensemble of models generated, the NA makes use of all the models, good and bad, generated during the first stage. The low and high misfits of the models are converted to high and low likelihoods, respectively. Assuming Gaussian-distributed errors on the measurements, the likelihood function is defined as:

$$
P(\mathbf{d} \mid \mathbf{m})=\exp \left[-\frac{1}{2}(\mathbf{d}-\mathbf{g}(\mathbf{m}))^{T} \mathbf{C}_{\mathbf{D}}^{-1}(\mathbf{d}-\mathbf{g}(\mathbf{m}))\right]
$$

where $\mathbf{g}(\mathbf{m})$ are phase velocity predictions calculated from model $\mathbf{m}$ and eq. 17 , and $\mathbf{C}_{\mathbf{D}}$ is the data covariance matrix. Here, the data covariance matrix is a diagonal matrix whose elements are given by the data variance. For dataset 1, the data variance was provided by Visser et al. (2008a) for each fundamental and higher mode. Because datasets 2 and 3 were not provided with uncertainties, we applied the same relative uncertainties as those determined by Visser et al. (2008a) to the Ekström (2011) phase velocities. For the group velocities, we imposed relative uncertainties twice as large as for the phase velocities as these data have typically larger error bars than phase velocities (Dr. Zhitu Ma, Personal communication).

The likelihood function $P(\mathbf{d} \mid \mathbf{m})$ is then used to obtain the posterior probability density functions (PPDFs) given by Bayes' theorem (Bayes and Price, 1763): 
$P(\mathbf{m} \mid \mathbf{d}) \propto \rho(\mathbf{m}) P(\mathbf{d} \mid \mathbf{m})$

where $P(\mathbf{m} \mid \mathbf{d})$ is a likelihood function representing the fit to the data, and $\rho(\mathbf{m})$ is the prior probability density distribution, defined here as uniform distributions around the inversion results. These PPDFs can be used to assess the robustness of the model parameters as they can be used to calculate the mean values and uncertainties of the model parameters, as well as covariance matrix and resolution matrix.

For a PPDF $P(\mathbf{m} \mid \mathbf{d})$, the posterior mean model for the $i t h$ parameter is given by the following integral performed over the model space (Sambridge, 1999b):

$$
<m_{i}>=\int m_{i} P(\mathbf{m} \mid \mathbf{d}) d \mathbf{m}
$$

The posterior variances of the model parameters can be obtained from the diagonals of the posterior model covariance matrix defined by:

$$
C_{i, j}=\int m_{i} m_{j} P(\mathbf{m} \mid \mathbf{d}) d(\mathbf{m})-<m_{i}><m_{j}>
$$

Note that the concepts of covariance matrix and the resolution kernels are linearized concepts, and are most useful if the PPDF has a single dominant peak, e.g. if the distribution is Gaussian. They are used here to get quantitative uncertainties on the model parameters, but one should keep in mind the limitations of these definitions. Because the null-space is included in the model space sampling, the model uncertainties inferred are more accurate than those resulting from regularized inversions which tend to underestimate posterior variance, especially in the presence of a large model the null-space (Trampert, 1998; Beghein and Trampert, 2003; Beghein, 2010).

The 1-D marginal distribution of a given model parameter $m_{i}$ can be obtained by integrating $P(\mathbf{m} \mid \mathbf{d})$ numerically over all other parameters (Sambridge, 1999b):

$$
M\left(m_{i}\right)=\int \ldots \int P(\mathbf{m} \mid \mathbf{d}) \prod_{k=1, k \neq i}^{d} d m_{k}
$$

where $d$ is the total number of model parameters. The shape and width of these 1-D marginals provide useful information on how well constrained a given parameter is and whether the model distribution is Gaussian, in which case the mean $<m_{i}>$ coincides with the peak of the distribution, i.e. the most likely value. In our study, they can also be used to calculate the distribution of possible LAB depths at a given grid cell, thereby providing quantitative uncertainties for each proxy employed. 


\section{Beghein, C., Xing, Z. and Goes, S.}

\section{RESULTS}

\subsection{Tomographic Models from Regularized Inversions}

In the following section, Model1_SVD, Model2_SVD, and Model3_SVD refer to the velocity and radial anisotropy models derived from the regularized inversion of dataset 1 , dataset 2 , and dataset 3 , respectively.

For all models, the number of independent parameters was six as determined by our SVD method (Matsu'ura and Hirata, 1982). The achieved variance reduction averaged over the study area was 98.6\% for Model1_SVD, 95\% for Model2_SVD, and 79\% for Model3_SVD. Synthetic tests showing the vertical resolution achieved with each dataset and the chosen regularization are represented in Figs. S1-S3. The depth resolution of datasets 1 and 2 for $V_{S}$ is between 50 and $70 \mathrm{~km}$ in the upper $150-200 \mathrm{~km}$, and closer to $30 \mathrm{~km}$ for dataset 3 . Neither dataset 1 nor dataset 2 performs particularly well for radial anisotropy. Dataset 3, however, should be able to recover an upper radial anisotropy layer of about $80 \mathrm{~km}$. These tests suggests that the inclusion of group velocity data helps improve the depth resolution of the models.

The $V_{S}$ models (Fig. 6) are overall consistent with one another and with previously published models, despite some differences between model amplitudes, which are likely due to differences in regularizations and in the phase velocity maps, which themselves result from a regularized inversion of path-averaged dispersion measurements. The inclusion of group velocity data did not affect the models significantly, although some differences are visible. For all models, we observe that mid ocean ridges are characterized by relatively low velocities at shallow depths whereas the rest of the region displays higher velocities than average. As depth increases, the relatively low $V_{S}$ becomes more widespread below the Pacific ocean, and the faster than average velocities that are likely the signature of a cool lithosphere disappear between $100 \mathrm{~km}$ and $150 \mathrm{~km}$. The difference between the area covered by positive velocity anomalies at $100 \mathrm{~km}$ depth in Model1_SVD and that of Model2_SVD or Model3_SVD suggests that the Ekström (2011) dataset favors a shallower high velocity lid than the Visser et al. (2008a) dataset.

While the velocity models are in general agreement with one another, the radial anisotropy models obtained exhibit significant discrepancies. Fig.7 7 displays maps of relative perturbations $\mathrm{dln} \xi$ in radial anisotropy (with $\xi=V_{S H}^{2} / V_{S V}^{2}$ ) with respect to anisotropic PREM at different depths. In PREM, the upper $220 \mathrm{~km}$ of the mantle are characterized by $V_{S H}>V_{S V}(\xi<0)$ and $V_{S H}=V_{S V}(\xi=1)$ at greater depths. Therefore, in our models $\operatorname{dn} \xi<0$ corresponds an even stronger $V_{S H}>V_{S V}$ signal than in PREM, and $\operatorname{dn} \xi>0$ means the total radial anisotropy $\xi$ is less strongly negative than in PREM. Locations with very large $\operatorname{dln} \xi>0$ anomalies may even correspond to $V_{S V}>V_{S H}$. Fig.7 
shows that $\operatorname{dln} \xi<0$ in the top $100 \mathrm{~km}$ and relatively uniformly across the Pacific for Model1_SVD, with decreasing amplitude as depth increases. We observe more lateral variations of $\xi$ in Model2_SVD, with more positive anomalies at $80 \mathrm{~km}$ and $100 \mathrm{~km}$ depth than in Model1_SVD. In both models, the strongest anomalies are negative. Model3_SVD has a strikingly different radial anisotropy signal than the other two models as it is negative only in the top $50 \mathrm{~km}$ and becomes positive over most of the study area at $80 \mathrm{~km}$ depth. These differences between models already suggest that the interpretation of existing radial anisotropy models in terms of the $\mathrm{LAB}$ is non-unique.

\subsection{Tomographic Models from the Model Space Search}

The application of the NA to each dataset yielded new distributions of tomographic models. With this approach, each model parameter is described by a PPDF instead of obtaining a single model for each dataset based on implicit regularization. The model space search was centered around the models obtained by SVD. The prior was a uniform distribution between -0.05 and 0.05 around the SVD results at all depths. For instance, if the i-th spline coefficient $\operatorname{dn} V_{S}^{i}($ Eq. 15 of the SVD model has a value of 0.04 , the prior distribution for this parameter is uniform between -0.01 and 0.09 . Similarly, if the i-th radial anisotropy spline coefficient $d \xi^{i}$ has a value of 0.05 in the SVD model, the model space search for this parameter ranges from 0 to 0.1 .

Figs. 8 and 9 represent the $V_{S}$ and $\xi$ mean models and their uncertainties. The mean models (labeled Model1_m, Model2_m, and Model3_m) and the uncertainty maps were constructed at each grid cell using the weighted mean and standard deviation of the PPDFs representing the $\operatorname{dnn} V_{S}^{i}$ and $\operatorname{dln} \xi^{i}$ spline coefficients (Eq. 16 ). The mean velocity models strongly resemble their regularized counterparts, except for Model2_m at $200 \mathrm{~km}$ depth which displays stronger amplitudes than Model2_SVD. Model3_m has slightly more negative velocity perturbations at $200 \mathrm{~km}$ than Model3_SVD. The average uncertainties are around 0.03 for $\operatorname{dn} \ln V_{S}$ in both Model1 $\_\mathrm{m}$ and Model2_m, and about 0.02 for Model3_m.

The mean anisotropy models are also close to the anisotropy models obtained by regularized inversions, the strongest differences being for dataset 2 at $100 \mathrm{~km}$ depth where Model2_m has more positive anomalies than Model2_SVD. In addition, the anisotropy anomalies of Model3_m at and below $80 \mathrm{~km}$ depth are slightly stronger than those of Model3_SVD. In Model2 $\mathrm{m}$, while the pattern of anisotropy at $50 \mathrm{~km}$ and $80 \mathrm{~km}$ depth are similar and display mostly negative anomalies of decreasing amplitude with depth, the pattern of anisotropy shows mostly positive perturbations and identical patterns at $100 \mathrm{~km}, 150 \mathrm{~km}$, and $200 \mathrm{~km}$ depth. In Model3_m, just like in Model3_SVD, there is a clear change from $d \ln \xi<0$ at $50 \mathrm{~km}$ and $d \ln \xi>0$ below. The average uncertainties are generally smaller than the mean values in all models. 


\section{Beghein, C., Xing, Z. and Goes, S.}

We also ran the NA around model PREM using dataset 1 as a test case to insure the results of Figs. 8 and 9 did not represent a local minimum, biased toward the SVD model (Fig. S4). These additional model space searches were slower to converge than those centered around the regularized inversion results, as expected when sampling a wider model space. The new models show slightly stronger amplitudes than those of Model1_m and some more lateral variations, but also larger uncertainties. This implies that the difference between models obtained by searching the model space around PREM or around the SVD model are likely not significant.

\subsection{LAB Depth Proxies and Uncertainties}

As discussed in section 11, several different seismological proxies can be used to characterize the LAB and determine its depth. Here, we focus on two proxies, one based on the S-wave velocity models and one based on the radial anisotropy models. The "seismic" LAB can be related to the change in velocity between a high-velocity lithosphere and a low-velocity asthenosphere. In previous studies, some authors chose to use the depth of the maximum negative velocity gradient (maximum of $\left.-\partial V_{S} / \partial z\right)$ to map the LAB depth (Burgos et al., 2014). Others (Bartzsch et al., 2011) defined the seismic LAB depth as the middle of the interval over which $V_{S}$ decreases since this drop may be sharp (over a few kilometres) or gradual (a few tens of kilometres). Here, we chose to employ the same proxy as Bartzsch et al. (2011). In addition, because the classical plate tectonics definition of the LAB is that it corresponds to a change in mechanical response, the LAB may be expected to be associated with a change in radial anisotropy similar to what has been found in azimuthal anisotropy models (e.g., Beghein et al. (2014)). We thus employed the depth of the largest positive $\xi$ gradient $(\partial \xi / \partial z)$ as another proxy for the LAB depth as in Burgos et al. (2014). We first calculated these proxies for each inverted dataset and at each grid cell using the $V_{S}$ and $\xi$ profiles obtained from our regularized inversions. We plotted them in map view (Fig. 10) and as a function of oceanic crust age (Fig. 11). The error bars displayed in Fig. 11 represent the range of values obtained across the study area for a given age but do not account for the non-uniqueness of the inverse problem.

With the $V_{S}$-defined LAB proxy, Model1_SVD yields a shallow LAB depth of $\sim 40 \mathrm{~km}$ at the East Pacific Rise (EPR) and the Cocos-Nazca spreading ridge, which deepens rapidly with age to a depth of $\sim 120 \mathrm{~km}$ over much of the Pacific (Fig. 10). The change in LAB depth with crustal age is also visible in Model2_SVD, but more gradual than in Model1_SVD, also starting at depths of around $40 \mathrm{~km}$ near the spreading centres and reaching $\sim 120 \mathrm{~km}$ below the oldest part of the plate. Model3_SVD yields a more uniform LAB depth of around $70 \mathrm{~km}$ across most of the study region only deepening to $\sim 120 \mathrm{~km}$ under the oldest part of the Pacific. One should, however, be reminded that the data fit provided by 
Model3_SVD was much lower than for the other two models and it may not therefore be a as reliable a model to use to determine the LAB depth.

Interestingly, for dataset 1 and dataset 2 , while the shallowest depths for the $V_{S}$-defined LAB proxy are located near the youngest parts of the ocean, these depths are greater than found in previous work (Burgos et al., 2014) and with a strong scatter for similar ages across the study area. This is also the case for older crustal ages, for which our results give generally deeper LAB depths than found by Burgos et al. (2014). We note that Burgos et al. (2014) employed a different $V_{S}$-defined LAB proxy than ours - they used the depth of the maximum negative $\partial V_{S} / \partial z-$ but we verified that using the same definition with our models does not affect our LAB depth estimates. While our larger LAB depth estimates could be the result of artefacts introduced by the various authors during the construction of their phase velocity maps, the fact that this is occurring with two datasets obtained with different measurement and inversion techniques suggests this signal is robust.

Both Figs. 10 and 11 demonstrate that the depth of largest positive $\partial \xi / \partial z$ is generally uniform across the Pacific with little-to-no age variation for all datasets employed, but the depth at which the maximum occurs does depend on the dataset. For Model1_SVD, this depth is around $80-90 \mathrm{~km}$, in agreement with results by Burgos et al. (2014). A similar depth is found for Model2_SVD, but this model also includes several locations with depths of about $20 \mathrm{~km}, 40 \mathrm{~km}$, and $>120 \mathrm{~km}$ scattered across the Pacific, with no clear age dependence. With group velocities (Model3_SVD), the depth proxy is shallower, around $55 \mathrm{~km}$, as already suggested by Fig. 9

Fig. 11 clearly shows that there is very little scatter in the depth of the maximum $\partial \xi / \partial z$, regardless of the dataset. This could be interpreted as evidence for an age-independent $\xi$-based proxy even if the depth of the LAB proxy itself depends on the dataset. Before drawing conclusions, it is however essential to look at the statistical distributions of all possible $\xi$ models to determine the robustness of these results. We were able to take advantage of the model space search approach employed to determine quantitative uncertainties on the depth of the two LAB depth proxies examined in this paper. For each grid cell, we drew random model spline coefficients $\operatorname{dn} V_{S}^{i}$ and $\operatorname{d} \ln \xi^{i}$ from their PDDFs to reconstruct a suite of $V_{S}(r)$ and $\xi(r)$ models, and for each of these models we calculated the depth of the LAB using our two proxies. This yielded distributions of LAB depths at each grid cell, from which a mean depth and standard deviation could be calculated. Those are represented in Fig. 12

As in the SVD inversions, the mean LAB depth resulting from the application of the NA to dataset 1 and using the velocity proxy is relatively shallow at ridges and increases toward the older ocean, and the values obtained in the youngest parts of the Pacific are deeper (around $60 \mathrm{~km}$ ) than usually expected from thermal cooling models The uncertainty map reveals signficant uncertainties with a standard deviation of around 10-15 km in the younger part of the Pacific and 15-20 km elsewhere The 


\section{Beghein, C., Xing, Z. and Goes, S.}

mean depth resulting from $\partial \xi / \partial z$ and dataset 1 is relatively uniform with values of $70-90 \mathrm{~km}$ and uncertainties of $5-25 \mathrm{~km}$.

With dataset 2, the middle of the velocity decrease interval yields a relatively deep LAB of about $50 \mathrm{~km}$ at the EPR and error bars of about $5 \mathrm{~km}$. The standard deviation increases to about $20 \mathrm{~km}$ toward the older plate. The mean value obtained from $\partial \xi / \partial z$ is around $80 \mathrm{~km}$ with an average error of $5-15 \mathrm{~km}$, similar to the results obtained from dataset 1 . Some lateral variations were also found, but they do not appear correlated with crustal age and because they are associated with larger uncertainties, they are likely not significant.

With dataset 3, the mean LAB depth based on the $V_{S}$ proxy displays larger values in the western Pacific than near ridges, and values close to $65 \mathrm{~km}$ over a large part of the Pacific. Uncertainties in this case are small $(<5 \mathrm{~km})$ over the northeastern Pacific, rising to $15-20 \mathrm{~km}$ in the northwestern part of the study region. The $\xi$ proxy is shallow over a broad section of the Pacific, similar to the SVD results, with values near $50 \mathrm{~km}$, and displays a few locations around 100-120 km in the oldest Pacific. Uncertainties on the $\xi$ proxy are under $5 \mathrm{~km}$ over much of the region, though several isolated locations have much larger error bars of 30-40 km.

The mean and standard deviation of these proxies are also represented as a function of crustal age in Fig. 13. Similar to Fig. 11, we plotted the mean and standard deviation for each proxy at every grid cell sorted by increasing seafloor age. Overall, the LAB depths obtained have uncertainties so large that the results from the three datasets are more compatible than it first appears by looking at the SVD results only. The uncertainties on the radial anisotropy models are sufficiently large that we cannot rule out the existence of a model that would yield an age-dependent $\partial \xi / \partial z$ compatible with the $V_{S^{-}}$ based proxy. We thus cannot readily conclude that the anisotropy and the velocity model proxies yield different LAB depths or are seeing different properties of the mantle as proposed by previous authors (Hier-Majumder and Drombosky, 2015; Hansen et al., 2016; Hedjazian et al., 2017).

\subsection{Discussion}

Our inversions confirm that oceanic lithosphere-asthenosphere shear wavespeed structure and proxies of the LAB vary systematically with crustal age. As discussed in the introduction, there are a number of outstanding questions about the variation in LAB depth with age. By comparing our $V_{S}$ models against synthetic velocity models, we evaluate the following: (1) Can lithosphere-asthenosphere velocities be explained purely with solid-state thermal effects? (2) Or is an additional signature of either the presence of melt or major-element depletion and dehydration of the mantle due to melting below the ridge required? (3) Can the results be used to discriminate between the end-member models of plate or half-space cooling of oceanic lithosphere with age? (4) Some previous work indicates that the 
potential temperature below the Pacific may be higher than below other oceans (Goes et al., 2013); do the surface-wave velocities corroborate this?

We computed synthetic seismic velocities for a set of halfspace and plate cooling models (Figs. 14 and S5). Thermal parameters were taken from McKenzie et al. (2005) (as described in Goes et al. (2012)), yielding half-space cooling and plate models that reasonably match global bathymetry and flow data as a function of cooling age, for mantle potential temperatures of $1315^{\circ} \mathrm{C}$. We also tested models with a hotter potential temperature of $1415^{\circ} \mathrm{C}$, which provides better fits to Pacific differential $P P-P$ and $S S-S$ travel times (Goes et al., 2013).

The temperature structures were converted to seismic velocities using the code PerPleX (Connolly, 2005) with the thermodynamic database from Xu et al. (2008) to compute elastic parameters and density and with additional correction for anelastic effects as in Goes et al. (2012). Two anelasticity models with different temperature- and pressure-dependence were tested: model $Q_{g}$, a combined experimental, empirical model similar to those that have been previously used to reconcile $V_{P}, V_{S}$ and heatflow observations in a range of tectonic settings (Goes et al., 2000, Shapiro and Ritzwoller, 2004), and model $Q_{J}$, based on experimental parameters from Jackson and Faul (2010). For model $Q_{J}$, grainsize is set to a constant $1 \mathrm{~cm}$ (see also discussion in Abers et al. (2014)). Synthetic models are shown for a frequency of 20s (Figs. 14 and S5) and comparisons are very similar when we account for the variation of velocity with frequency.

For composition, all models include the effect of major element depletion of the mantle residue due to melting, implemented as a gradient from a peridotitic composition below the depth where (hydrous) melting starts to a fully harzburgitic composition after $20 \%$ melt has been extracted. The melting conditions depend on potential temperature and whether models are assumed to be dry, damp $\left(1000 \mathrm{H} / 10^{6} \mathrm{Si}\right)$ or wet $\left(3000 \mathrm{H} / 10^{6} \mathrm{Si}\right)$ and are taken from the dynamic spreading models computed in Goes et al. (2012). The presence of water is assumed to affect attenuation (see Goes et al. (2012)), although the presence of water itself may not actually lower attenuation, but rather redox conditions could be responsible (Cline et al.2018), which, at least in subduction settings, may correlate with water content (Kelley and Cottrell, 2009). Oceanic mantle is on average likely somewhat damp (Hirth and Kohlstedt, 1996). We also consider dry models, because of the above arguments about sensitivity of Q to water (Cline et al.2018) and, in addition, Abers et al. (2014) argue that hydrous weakening effects on attenuation are offset by enhanced grain growth. For the models that assume water is present, dehydration occurs between the depths where the hydrous solidus and dry solidus are crossed ( 85 and $52 \mathrm{~km}$ respectively for a damp $1315^{\circ} \mathrm{C}$ mantle). None of the synthetic models incorporate any effects of the presence of melt, which would lower velocities, at least directly below the ridge, and likely enhance attenuation (Eilon and Abers, 2017). Note that enhanced attenuation due to hydration plus 
decreased attenuation upon dehydration during melting might explain the double low-velocity zone observed in regional surface-wave tomography below the East Pacific Rise (Dunn and Forsyth, 2003), in which case the lower zone occurs in hydrated, solid-state asthenosphere and the upper zone is due to the presence of melt in a largely dehydrated mantle (Goes et al., 2012). As we will show below, a process that lowers attenuation (increases Q) during melting would also help explain the Pacific-wide shear wave velocities with age.

We performed resolution tests using these synthetic $V_{S}$ models as input with dataset 2, and determined that our regularized inversion scheme was able to recover the input models relatively well, though a slight deepening of the low velocity zone is visible in some of the output models (Figs. S6 to S9). In Fig. 15, we compare synthetic $V_{S}$ depth profiles for model $Q_{g}$ for different crustal ages with the velocity profiles resulting from regularized inversion of dataset 2 for the same ages. Equivalent figures for model $Q_{g}$ with dataset 1 and dataset 3 and for model $Q_{J}$ with dataset 2 can be found in the supplementary material (Figs. S10 to S12). Tomographic imaging and uncertainties in the tomographic solutions are expected to cause differences between synthetic and tomographic profiles even if structures would be identical. However, bearing in mind these uncertainties, several differences between the synthetic and tomographic profiles are noteworthy:

1) The models without any melt but with hydration effects on Q predict an asthenosphere which is easily slow enough to match the tomographic results. Dry models are too fast and predict velocity minima that are significantly too shallow below younger lithosphere. Lower asthenospheric velocities could of course also be obtained in a dry asthenosphere by adding an effect of melt below lithosphere of at least all ages $\leq 70$ Myr. However, melt would not help with the next point.

2) The effects on attenuation of dehydration by melting deepen the velocity minimum below young lithosphere and improve the fit of velocity-age trends. An additional velocity-lowering effect of the presence of melt is expected locally below the ridge, but would not be observable with the type of data used here (Goes et al., 2012). If the asthenospheric velocity minimum were the result of the presence of melt instead of being associated with hydration, it is not clear why attenuation would be reduced and velocities increased above depths where significant melting starts below the ridge.

3) The profiles in Fig. 15 clearly show that the differences in velocity for different ages in our tomographic model persist to greater depths than the plate thickness in the plate cooling models. Halfspace cooling models predict differences in $V_{S}$ down to depths of $\sim 175 \mathrm{~km}$, which matches our tomographic results better. From this we conclude that our velocity models are best represented by halfspace type cooling .

4) Wetter and/or hotter models move the velocity minima to larger depths, yielding the best agreement with the depths from the tomographic models. The hot and wet models also predict lower 
velocities below the asthenosphere than found in the tomographic inversion, although these differences may at least in part be affected by uncertainties in the depth-dependence of attenuation and by limited resolutions of the data at depths exceeding $200 \mathrm{~km}$.

In Fig. 16 we compare our $V_{S}$-defined LAB depth proxy determined from our tomographic models and from the synthetic velocity models of Goes et al. (2012) calculated for HSC and attenuation model $Q_{g}$. We focus on HSC models from here on since they explain our $V_{S}$ models better than plate models, as discussed above. We see that, for all three datasets and the regularized inversions, the LAB depth for the youngest oceans is best explained by a damp and hot $\left(T_{m}=1415^{\circ} \mathrm{C}\right)$ mantle. The main reason for this preference is that the higher degree of melting predicted by this model leads to a thicker dehydrated layer, thus depressing the asthenospheric velocity minimum. All tomographic models, including those within the error bars obtained with the NA, prefer structures with velocity minima on the deeper end of those our forward thermal models predict. For attenuation model $Q_{J}$ (Fig. S13), both a hot $\left(T_{m}=1415^{\circ} \mathrm{C}\right)$ and a $T_{m}=1315^{\circ} \mathrm{C}$ wet model predict deep enough velocity minima. However, the increase of velocity with depth below $200 \mathrm{~km}$ is better matched by the pressure-dependence in model $Q_{g}$ than model $Q_{J}$, and this pressure dependence largely offsets the deepening of the velocity minimum for the $1315^{\circ} \mathrm{C}$ wet model. Absolute velocities are too uncertain to discriminate between mantle temperatures. However, the comparisons indicate a strong preference for structures in which dehydration leads to a less attenuating layer down to about $80 \mathrm{~km}$ depth overlying a more attenuating asthenosphere, as predicted by the hotter mantle models.

Although the tomographic models resulting from datasets 1 and 3 are not matched as well as by the synthetic model trends as the models obtained from dataset 2 (Fig. 16), all datasets are consistent with a continued trend of lithospheric cooling until old ages, and all three datasets indicate LAB-age gradients which are more compatible with those from the models where depletion by melting leads to decreased attenuation. Our results therefore suggest no significant fractions of melt are needed to explain the shear-wave velocities (although uncertainties are large enough that some amount of melt could be present). This is consistent, as pointed out by Goes et al. (2012), with petrological constraints predicting that melt is efficiently removed at asthenospheric depths (Asimow et al., 2004). This would indicate that other mechanisms than melt may be necessary to explain or at least contribute to the existence of the Gutenberg discontinuity, e.g. associated with fabrics that form due to the melting that occurs below the ridge (Hansen et al., 2016, Hedjazian et al., 2017), which is also supported by the azimuthal anisotropy results of Beghein et al. (2014). 


\section{Beghein, C., Xing, Z. and Goes, S.}

\section{CONCLUSIONS}

We applied a Bayesian approach to various surface wave datasets to constrain radial anisotropy and shear-wave velocities under the Pacific plate and determine the depth of the LAB using various proxies calculated from our tomographic models. Our results show that, while regularized inversion results can yield different LAB depth depending on the dataset employed, the uncertainties on the models are large and the resulting LAB depth proxies are, in fact, compatible with one another. Radial anisotropy models are poorly constrained and we cannot conclude that they require different LAB depth estimates than isotropic velocity models. In addition, testing our velocity models against the synthetic models of Goes et al. (2012) for half space and plate models showed that our results are best explained by half space cooling models that include a decrease in attenuation due dehydration by melting at the ridge than by dry models or plate models. The imaged depths of the asthenospheric velocity minimum are deeper than predicted for a dry mantle and most consistent with the depths predicted for a damp, $1415^{\circ} \mathrm{C}$ mantle where higher degrees of melting lead to a thicker dehydrated layer than in a $1315^{\circ} \mathrm{C}$ mantle. The asthenospheric low velocities could also be explained with the presence of melt, but the observed velocity-age trends are not easily explained by melt effects. In addition, given the large uncertainties in the velocity- and anisotropy-based proxies for all datasets, we cannot reject the hypothesis that the different proxies are all compatible with a thermally controlled, i.e., age-dependent LAB.

\section{ACKNOWLEDGMENTS}

We wish to thank Göran Ekström, Karin Visser and Jeannot Trampert for making the surface wave velocity maps freely available online at http://www.geo.uu.nl/ jeannot/My_web_pages/Downloads . html and http://www.ldeo.columbia.edu/ ekstrom/, and Malcom Sambridge for sharing his Neighbourhood Algorithm. Partial derivatives were calculated using program MINEOS (available on the CIG website at http://www.geodynamics.org/), and figures were made using the Generic Mapping Tool. Calculations were made on C.B.'s computer cluster, which was funded by NSF grant \#0949255.

\section{REFERENCES}

Abers, G. A., K. M. Fischer, G. Hirth, D. A. Wiens, T. Plank, B. K. Holtzman, C. McCarthy, and E. Gazel (2014), Reconciling mantle attenuation- temperature relationships from seismology, petrology, and laboratory measurements, Geochem. Geophys. Geosys. 15, doi10.1002/ 2014GC005444. 
Auer, L., Boschi, L., Becker, T., Nissen-Meyer, T., \& Giardini, D., 2014. Savani: A variable resolution wholemantle model of anisotropic shear velocity variations based on multiple data sets, J. geophys. Res., 119(4), 3006-3034, doi10.1002/2013JB010773.

Amante, C., and B. Eakins (2009), Etopo1 1 arc-minute global relief model: Procedures, data sources and analysis, NOAA Technical Memorandum NESDIS NGDC-24, National Geophysical Data Center, NOAA.

Anderson, D. L., and C. Sammis (1970), Partial melting in the upper mantle, Phys. Earth Planet. Inter., 3, 41-50, doi10.1016/0031-9201(70)90042-7.

Asimow, P. D., Dixon, J. E., \& Langmuir, C. H. , 2004. A hydrous melting and fractionation model for midocean ridge basalts: Application to the Mid-Atlantic Ridge near the Azores, Geochem., Geophys., Geosyst., 5, doi10.1029/2003GC000568.

Babuška, V., and M. Cara (1991), Seismic Anisotropy in the Earth, Modern Approaches in Geophysics, vol. 10, Springer Netherlands, Dordrecht.

Bagley, B., and J. Revenaugh (2008), Upper mantle seismic shear discontinuities of the Pacific, J. geophys. Res., 113, B12, 301, doi10.1029/2008JB005692.

Bartzsch, S., S. Lebedev, and T. Meier (2011), Resolving the lithosphere-asthenosphere boundary with seismic Rayleigh waves, Geophys. J Int., 186(3), 1152-1164.

Bayes, M., and M. Price (1763), An essay towards solving a problem in the doctrine of chances. By the late rev. mr. bayes, frs communicated by mr. Price, in a letter to John Canton, amfrs, Philosophical Transactions of the Royal Society of London, pp. 370-418, doi10.1098/rstl.1763.0053.

Becker, T. W., C. P. Conrad, A. J. Schaeffer, and S. Lebedev (2014), Origin of azimuthal seismic anisotropy in oceanic plates and mantle, Earth Planet. Sci. Lett., 401, 236-250, doi10.1016/j.eps1.2014.06.014.

Beghein, C. (2010), Radial anisotropy and prior petrological constraints: A comparative study, J. geophys. Res., 115(B3), doi10.1029/2008jb005842.

Beghein, C., and J. Trampert (2003), Robust normal mode constraints on inner-core anisotropy from model space search, Science, 299(5606), 552-555, doi10.1126/science.1078159.

Beghein, C., J. Resovsky, and R. D. Van Der Hilst (2008), The signal of mantle anisotropy in the coupling of normal modes, Geophys. J Int., 175(3), 1209-1234.

Beghein, C., K. Yuan, N. Schmerr, and Z. Xing (2014), Changes in seismic anisotropy shed light on the nature of the Gutenberg discontinuity, Science, 343(6176), 1237-1240, doi10.1126/science.1246724.

Behn, M., G. Hirth, and J. R. Elsenbeck II (2009), Implications of grain size evolution on the seismic structure of the oceanic upper mantle, Earth Planet. Sci. Lett., 282(1-4), 178-189, doi10.1016/j.eps1.2009.03.014.

Boschi, L., and G. Ekström (2002), New images of the Earth's upper mantle from measurements of surface wave phase velocity anomalies, J. geophys. Res., 107(B4), doi10.1029/2000jb000059.

Bozdag, E., and J. Trampert (2008), On crustal corrections in surface wave tomography, Geophys. J Int., 172, 1066-1082, doi10.1111/j.1365-246X.2007.03690.x.

Burgos, G., J.-P. Montagner, E. Beucler, Y. Capdeville, A. Mocquet, and M. Drilleau (2014), Oceanic lithosphere-asthenosphere boundary from surface wave dispersion data, J. geophys. Res., 119(2), 1079- 
1093, doi10.1002/2013jb010528.

Chang, S.-J., A. M. Ferreira, J. Ritsema, H. J. van Heijst, and J. H. Woodhouse (2014), Global radially anisotropic mantle structure from multiple datasets: a review, current challenges, and outlook, Tectonophysics, 617, 1-19, doi10.1016/j.tecto.2014.01.033.

Chave, A. D., R. P. Von Herzen, K. A. Poehls, and C. S. Cox (1981), Electromagnetic induction fields in the deep ocean north-east of Hawaii: implications for mantle conductivity and source fields, Geophys. J Int., 66(2), 379-406, doi10.1111/j.1365-246X.1981.tb05961.x.

Cline II, C. J., U. H. Faul, E. C. David, A. J. Berry, and I. Jackson (2018), Redox-influenced seismic properties of upper-mantle olivine, Nature 555, 355358, doi10.1038/nature25764.

Connolly, J.A.D. (2005), Computation of phase equilibria by linear programming: A tool for geodynamic modeling and its applicationto subduction zone decarbonation, Earth Planet. Sci. Lett., 236, 524-541, doi10.1016/j.epsl.2005.04.033.

Courtier, A., and G. Leahy (2011), Joint receiver function/scs reverberation analysis for examining discontinuity structure beneath ocean islands, Bull. seism. Soc. Am., 101(2), 908-914, doi10.1785/0120100209.

Debayle, E., and Y. Ricard (2013), Seismic observations of large-scale deformation at the bottom of fast-moving plates, Earth Planet. Sci. Lett., 376, 165-177, doi10.1016/j.epsl.2013.06.025.

Dunn, R. A., and D. W. Forsyth (2003), Imaging the transition between the region of mantle melt generation and the crustal magma chamber beneath the southern East Pacific Rise with short-period Love waves, $J$. geophys. Res., 108, B7, 2352, doi10.1029/2002JB002217.

Dziewonski, A. M., and D. L. Anderson (1981), Preliminary reference Earth model, Phys. Earth Planet. Inter., 25(4), 297-356, doi10.1016/0031-9201(81)90046-7.

Eaton, D. W., F. Darbyshire, R. L. Evans, H. Grütter, A. G. Jones, and X. Yuan (2009), The elusive lithosphereasthenosphere boundary (LAB) beneath cratons, Lithos, 109(1), 1-22, doi10.1016/j.lithos.2008.05.009.

Eilon, Z. C., and G. A. Abers (2017), High seismic attenuation at a mid-ocean ridge reveals the distribution of deep melt, Science Advances, 3(5), doi10.1126/sciadv.1602829.

Ekström, G. (2011), A global model of Love and Rayleigh surface wave dispersion and anisotropy, 25-250 s, Geophys. J Int., 187, 1668-1686, doi10.1111/j.1365-246X.2011.05225.x.

Faul, U. H., and I. Jackson (2005), The seismological signature of temperature and grain size variations in the upper mantle, Earth Planet. Sci. Lett., 234(1-2), 119-134, doi10.1029/2001JB001225.

Ferreira, a. M. G., J. H. Woodhouse, K. Visser, and J. Trampert (2010), On the robustness of global radially anisotropic surface wave tomography, J. geophys. Res., 115(B4), 1-16, doi10.1029/2009jb006716.

Filloux, J. H. (1980), Magnetotelluric soundings over the northeast pacific may reveal spatial dependence of depth and conductance of the asthenosphere, Earth Planet. Sci. Lett., 46(2), 244-252, doi10.1016/0012821X(80)90010-2.

Fischer, K. M., H. A. Ford, D. L. Abt, and K. Rychert (2010), The lithosphere-asthenosphere boundary, Ann. Rev. Earth Planet Sci., 38(1), 551-575, doi10.1146/annurev-earth-040809-152438.

Forsyth, D. W., et al. (1998), Imaging the deep seismic structure beneath a mid-ocean ridge: The MELT experi- 
ment, Science, 280, 1215-1218, doi10.1126/science.280.5367.1215.

French, S., V. Lekic, and B. Romanowicz (2013), Waveform tomography reveals channeled flow at the base of the oceanic asthenosphere, Science, $\mathbf{3 4 2}$ (6155), 227-230.

Gaherty, J. B., T. H. Jordan, and L. S. Gee (1996), Seismic structure of the upper mantle in a central pacific corridor, J. geophys. Res., 101, 22,291-22,309, doi10.1029/96JB01882.

Goes, S., R. Govers, and P. Vacher (2000), Shallow upper mantle temperatures under Europe from P and S wave tomography, J. geophys. Res., 105, B5, 11,15311,169, doi10.1029/1999JB900300.

Goes, S., . Armitage, J, N. Harmon, A. Smith, and R. Huismans (2012), Low seismic velocities below mid-ocean ridges: Attenuation versus melt retention, J. geophys. Res., 117, B12, doi10.1029/2012JB009637.

Goes, S., .C. M. Eakin, J. Ritsema (2013), Lithospheric cooling trends and deviations in oceanic PP-P and SS-S differential traveltimes, J. geophys. Res., 118, doi10.1002/jgrb.50092.

Gripp, A. E., and R. G. Gordon (1990), Current plate velocities relative to the hotspots incorporating the NUVEL1 global plate motion model, Geophys. Res. Lett., 17(8), 1109-1112, doi10.1029/GL017i008p01109.

Gripp, A. E., and R. G. Gordon (1992), Young tracks of hotspots and current plate velocities, Geophys. J Int., 150(2), 321-361, doi10.1046/j.1365-246X.2002.01627.x.

Hansen, L. N., C. Qi, and J. M. Warren (2016), Olivine anisotropy suggests Gutenberg discontinuity is not the base of the lithosphere, Proceedings of the National Academy of Sciences, 113, 10,503-10,506, doi10.1073/pnas.1608269113.

Harmon, N., D. W. Forsyth, and D. S. Weeraratne (2009), Thickening of young Pacific lithosphere from highresolution Rayleigh wave tomography: A test of the conductive cooling mode, Earth Planet. Sci. Lett., 278, 96-106, doi10.1016/j.eps1.2008.11.025.

Hedjazian, N., F. Garel, D. R. Davies, and E. Kaminski (2017), Age-independent seismic anisotropy under oceanic plates explained by strain history in the asthenosphere, Earth Planet. Sci. Lett., 460, 135-142, doi10.1016/j.epsl.2016.12.004.

Hier-Majumder, S., and T. Drombosky (2015), Development of anisotropic contiguity in deforming partially molten aggregates: 2. Implications for the lithosphere-asthenosphere boundary, J. geophys. Res., 120, 764777, doi10.1002/2014JB011454.

Hirschmann, M.M. (2010), Partial melt in the oceanic low velocity zone, Phys. Earth Planet. Inter., 179, 60-71, doi10.1016/j.pepi.2009.12.003.

Hirth, G., and D. L. Kohlstedt (1996), Water in the oceanic upper mantle: implications for rheology, melt extraction and the evolution of the lithosphere, Earth Planet. Sci. Lett., 144(1), 93-108, doi10.1016/0012821X(96)00154-9.

Jackson, I., and U. H. Faul (2010), Grainsize-sensitive viscoelastic relaxation in olivine: Towards a robust laboratory-based model for seismological application, Phys. Earth Planet. Inter., 183(1-2), 151-163, doi10.1016/j.pepi.2010.09.005.

Karato, S.-i. (2012), On the origin of the asthenosphere, Earth Planet. Sci. Lett., 321, 95-103, doi10.1016/j.epsl.2012.01.001. 
Karato, S.-i., and H. Jung (1998), Water, partial melting and the origin of seismic low velocity and high attenuation zone in the upper mantle, Earth Planet. Sci. Lett., 157, 193-207, doi10.1016/S0012-821X(98)00034-X.

Karato, S.-i., H. Jung, I. Katayama, and P. Skemer (2008), Geodynamic significance of seismic anisotropy of the upper mantle: New insights from laboratory studies, Ann. Rev. Earth Planet Sci., 36, 59-95.

Kawakatsu, H., P. Kumar, Y. Takei, and M. Shinohara (2009), Seismic evidence for sharp lithosphereasthenosphere boundaries of oceanic plates, Science, 324(5926), 499-502, doi10.1126/science.1169499.

Kelley, K. A., and E. Cottrell (2009), Water and the Oxidation State of Subduction Zone Magmas, Science 325, 605-607, doi10.1126/science.1174156.

Komatitsch, D., and J. Tromp (2002a), Spectral-element simulations of global seismic wave propagation validation, Geophys. J Int., 149, 390-412.

Komatitsch, D., and J. Tromp (2002b), Spectral-element simulations of global seismic wave propagation - ii. Three-dimensional models, oceans, rotation and self-gravitation, Geophys. J Int., 150, 303-318.

Kreemer, C. (2009), Absolute plate motions constrained by shear wave splitting orientations with implications for hot spot motions and mantle flow, J. geophys. Res., 114(B10405), doi10.1029/2009JB006416.

Kustowski, B., A. M. Dziewonski, and G. Ekström (2007), Nonlinear Crustal Corrections for Normal-Mode Seismograms, Bull. seism. Soc. Am., 97(5), 1756-1762, doi10.1785/0120070041.

Laske, G., G. Masters, Z. Ma, and M. Pasyanos (2013), Update on CRUST1.0 - A 1-degree Global Model of Earth's Crust, in EGU General Assembly Conference Abstracts, EGU General Assembly Conference Abstracts, vol. 15, p. 2658.

Lekić, V., and B. Romanowicz (2011), Inferring upper-mantle structure by full waveform tomography with the spectral element method, Geophys. J Int., 185(2), 799-831.

Lizarralde, D., A. Chave, G. Hirth, and A. Schultz (1995), Northeastern Pacific mantle conductivity profile from long-period magnetotelluric sounding using Hawaii-to-California submarine cable data, J. geophys. Res., 100(B9), 17,837-17,854, doi10.1029/95JB01244.

Love, A. E. H. (1927), A treatise on the mathematical theory of elasticity, New York, Dover.

Lundstrom, C.C. (2003), Uranium-series disequilibria in mid-ocean ridge basalts: Observations and models of basalt genesis, Rev. Mineral. Geochem., 52, 175-214, doi10.2113/0520175.

Ma, Z., and C. Dalton (2017), Evolution of the lithosphere in the Indian ocean from combined earthquake and ambient noise tomography, J. geophys. Res., 121, 1-18, doi10.1002/2016JB013516.

Maggi, A., E. Debayle, K. Priestley, and G. Barruol (2006), Multimode surface waveform tomography of the Pacific ocean: a closer look at the lithospheric cooling signature, Geophys. J Int., 166(3), 1384-1397, doi10.1111/j.1365-246x.2006.03037.x.

Marone, F., and B. Romanowicz (2007), Non-linear crustal corrections in high-resolution regional waveform seismic tomography, Geophys. J Int., 170(1), 460-467, doi10.1111/j.1365-246x.2007.03399.x.

Matsu'ura, M., and N. Hirata (1982), Generalized least-squares solutions to quasi-linear inverse problems with a priori information, Journal of Physics of the Earth, 30(6), 451-468, doi10.4294/jpe1952.30.451.

McKenzie, D., J. Jackson, and J. Priestley (2005), Thermal structure of oceanic and continental lithosphere, 
Earth Planet. Sci. Lett., 233, 337-349, doi10.1016/j.eps1.2005.02.005.

Montagner, J.-P., and D. L. Anderson (1989), Petrological constraints on seismic anisotropy, Phys. Earth Planet. Inter., 54(1-2), 82-105, doi10.1016/0031-9201(89)90189-1.

Montagner, J.-P., and H.-C. Nataf (1986), A simple method for inverting the azimuthal anisotropy of surface waves, J. geophys. Res., 91(B1), 511-520, doi10.1029/jb091ib01p00511.

Naif, S., K. Key, S. Constable, and R. L. Evans (2013), Melt-rich channel observed at the lithosphereasthenosphere boundary, Nature, 495(7441), 356-359, doi10.1038/nature11939.

Nettles, M., and A. M. Dziewoński (2008), Radially anisotropic shear velocity structure of the upper mantle globally and beneath north America, J. geophys. Res., 113(B2), doi10.1029/2006jb004819.

Nishimura, C. E., and D. W. Forsyth (1989), The anisotropic structure of the upper mantle in the Pacific, Geophys. J., 96(2), 203-229, doi10.1111/j.1365-246X.1989.tb04446.x.

Olugboji, T. M., J. Park, S.-i. Karato, and M. Shinohara (2016), Nature of the seismic lithosphere-asthenosphere boundary within normal oceanic mantle from high-resolution receiver functions, Geochem., Geophys., Geosyst., 17(4), 1265-1282, doi10.1002/2015GC006214.

Panning, M., and B. Romanowicz (2006), A three-dimensional radially anisotropic model of shear velocity in the whole mantle, Geophys. J Int., 167(1), 361-379, doi10.1111/j.1365-246x.2006.03100.x.

Panning, M. P., V. Lekić, and B. a. Romanowicz (2010), Importance of crustal corrections in the development of a new global model of radial anisotropy, J. geophys. Res., 115(B12), B12,325, doi10.1029/2010jb007520.

Parisi, L., and A. M. Ferreira (2016), Empirical assessment of the validity limits of the surface wave full ray theory using realistic 3-D Earth models, Geophys. J Int., 205, 1, doi10.1093/gji/ggw005.

Park, J., and Y. Yu (1992), Anisotropy and coupled free oscillations - simplified models and surface-wave observations, Geophys. J. R. astr. Soc., 110(3), 401-420, doi10.1111/j.1365-246X.1992.tb02082.x.

Parsons, B., and J. G. Sclater (1977), An analysis of the variation of ocean floor bathymetry and heat flow with age, J. geophys. Res., 82(5), 803-827, doi10.1029/JB082i005p00803.

Revenaugh, J., and T. H. Jordan (1991), Mantle layering from ScS reverberations: 3. The upper mantle, J. geophys. Res., 96(B12), 19,781-19,810, doi10.1029/91JB01487.

Ritzwoller, M. H., N. M. Shapiro, and S.-J. Zhong (2004), Cooling history of the Pacific lithosphere, Earth Planet. Sci. Lett., 226(1), 69-84, doi10.1016/j.eps1.2004.07.032.

Rodi, W., P. Glover, M. Li, and S. Alexander (1975), A fast, accurate method for computing group-velocity partial derivatives for Rayleigh and Love modes, Bull. seism. Soc. Am., 65(5), 1105-1114.

Röhm, A.H.E., R. K. Snieder, S. Goes, and J. Trampert (2000), Thermal structure of continental upper mantle inferred from S wave velocity and surface heat flow, Earth Planet. Sci. Lett., 181, 395-407, doi:10.1016/S0012-821X(00)00209-0.

Rubin, K. H., I. Van der Zander, M. C. Smith, and E. C. Bergmanis (2005), Minimum speed limit for ocean ridge magmatism from ${ }^{210} \mathrm{~Pb}-{ }^{226} \mathrm{Ra}-{ }^{230} \mathrm{Th}$ disequilibria, Nature, 437, 534-538, doi10.1038/nature03993.

Rychert, C., and P. M. Shearer (2011), Imaging the lithosphere-asthenosphere boundary beneath the Pacific using ss waveform modeling, J. geophys. Res., 116(B07307), doi10.1029/2010JB008070. 
Sakamaki, T., A. Suzuki, E. Ohtani, H. Terasaki, S. Urakawa, Y. Katayama, K. i. Funakoshi, Y. Wang, J. W. Hernlund, and M. D. Ballmer (2013), Ponded melt at the boundary between the lithosphere and asthenosphere, Nature Geoscience, 6(12), 1041-1044, doi10.1038/ngeo1982.

Sambridge, M. (1999a), Geophysical inversion with a neighbourhood algorithm-I. Searching a parameter space, Geophys. J Int., 138(2), 479-494, doi10.1046/j.1365-246x.1999.00876.x.

Sambridge, M. (1999b), Geophysical inversion with a neighbourhood algorithm-II. Appraising the ensemble, Geophys. J Int., 138(3), 727-746, doi10.1046/j.1365-246x.1999.00900.x.

Schaeffer, A. J., S. Lebedev, and T. W. Becker (2016), Azimuthal seismic anisotropy in the Earth's upper mantle and the thickness of tectonic plates, Geophys. J Int., 207, 901-933, doi10.1093/gji/ggw309.

Schmerr, N. (2012), The Gutenberg discontinuity: Melt at the lithosphere-asthenosphere boundary, Science, 335(6075), 1480-1483, doi10.1126/science.1215433.

Shankland, T. J., R. J. O'Connell, and H. S. Waff (1981), Geophysical constraints on partial melt in the upper mantle, J. geophys. Res., 19(3), 394-406, doi10.1029/RG019i003p00394.

Shapiro, N. M., and M. H. Ritzwoller (2004), Thermodynamic constraints on seismic inversions, Geophys. J Int., 157, 11751188, doi10.1111/ j.1365-246X.2004.02254.X.

Smith, D., M. H. Ritzwoller, and N. Shapiro (2004), Stratification of anisotropy in the Pacific upper mantle, J. geophys. Res., 109(B11), doi10.1029/2004JB003200.

Stein, C. A., and S. Stein (1992), A model for the global variation in oceanic depth and heat flow with lithospheric age, Nature, 359(6391), 123-129, doi10.1038/359123a0.

Steinberger, B., and T. Becker (2016), A comparison of lithospheric thickness models, Tectonophysics, doi10.1016/j.tecto.2016.08.001, in press.

Stixrude, L., and C. Lithgow-Bertelloni (2005), Mineralogy and elasticity of the oceanic upper mantle: Origin of the low-velocity zone, J. geophys. Res., , 110, B03204, doi10.1029/2004JB002965.

Stracke, A., B. Bourdon, and D. McKenzie (2006), Melt extraction in the Earths mantle: Constraints from UTh-Pa-Ra studies in oceanic basalts, Earth Planet. Sci. Lett., , 244, 97-112, doi10.1016/j.eps1.2006.01.057.

Takeuchi, H., and M. Saito (1972), Seismic surface waves, in Methods in computational physics, vol. 11, edited by B. Bolt, pp. 217-295, Academic Press, New York.

Tharimena, S., C. Rychert, N. Harmon, and P. White (2017a), Imaging Pacific lithosphere seismic discontinuities - Insights from SS precursor modeling, J. geophys. Res., 122, doi10.1002/2016JB013526.

Tharimena, S., C. Rychert, and N. Harmon (2017b), A unified continental thickness from seismology and diamonds suggests a melt-defined plate, Science, 357(6351), 580-583, doi10.1126/science.aan0741.

Trampert, J. (1998), Global seismic tomography: the inverse problem and beyond, Inverse Problems, 14(3), 371, doi10.1088/0266-5611/14/3/002.

van Wijk, J., J. Van Hunen, and S. Goes (2008), Small-scale convection during continental rifting: Evidence from the Rio Grande rift, Geology, 36(7), 575578, doi10.1130/G24691A.1.

Visser, K., J. Trampert, and B. L. N. Kennett (2008a), Global anisotropic phase velocity maps for higher mode Love and Rayleigh waves, Geophys. J Int., 172(3), 1016-1032, doi10.1111/j.1365-246x.2007.03685.x. 
Visser, K., J. Trampert, S. Lebedev, and B. Kennett (2008b), Probability of radial anisotropy in the deep mantle, Earth Planet. Sci. Lett., 270(3-4), 241-250, doi10.1016/j.eps1.2008.03.041.

Woodhouse, J. H., and F. A. Dahlen (1978), The effect of a general aspherical perturbation on the free oscillations of the Earth, Geophys. J. R. astr. Soc., , 53(2), 335-354, doi10.1111/j.1365-246X.1978.tb03746.x.

Xing, Z., and C. Beghein (2015), A bayesian approach to assess the importance of crustal corrections in global anisotropic surface wave tomography, Geophys. J Int., 203(3), 1832-1846, doi10.1093/gji/ggv401.

Xu, W., C. Lithgow-Bertelloni, L. Stixrude, and J. Ritsema (2008), The effect of bulk composition and temperature on mantle seismic structure, Earth Planet. Sci. Lett., 275, 70-79, doi10.1016/j.eps1.2008.08.012.

Yuan, K., and C. Beghein (2014), Three-dimensional variations in Love and Rayleigh wave azimuthal anisotropy for the upper $800 \mathrm{~km}$ of the mantle, J. geophys. Res., 119(4), 3232-3255, doi10.1002/2013JB010853.

Zhang, S., and S.-i. Karato (1995), Lattice preferred orientation of olivine aggregates deformed in simple shear, Nature, 375, 774-777. 
Beghein, C., Xing, Z. and Goes, S.
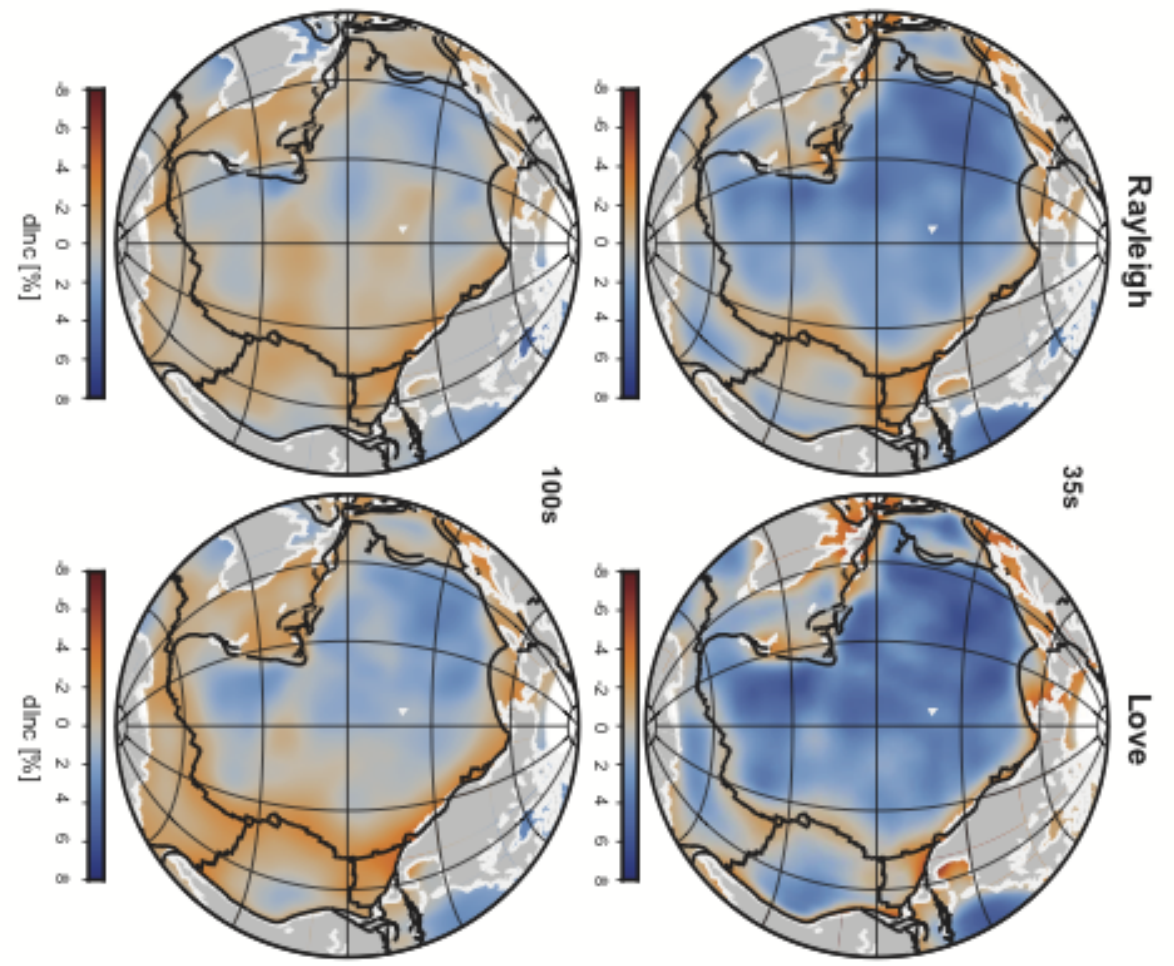

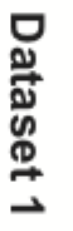
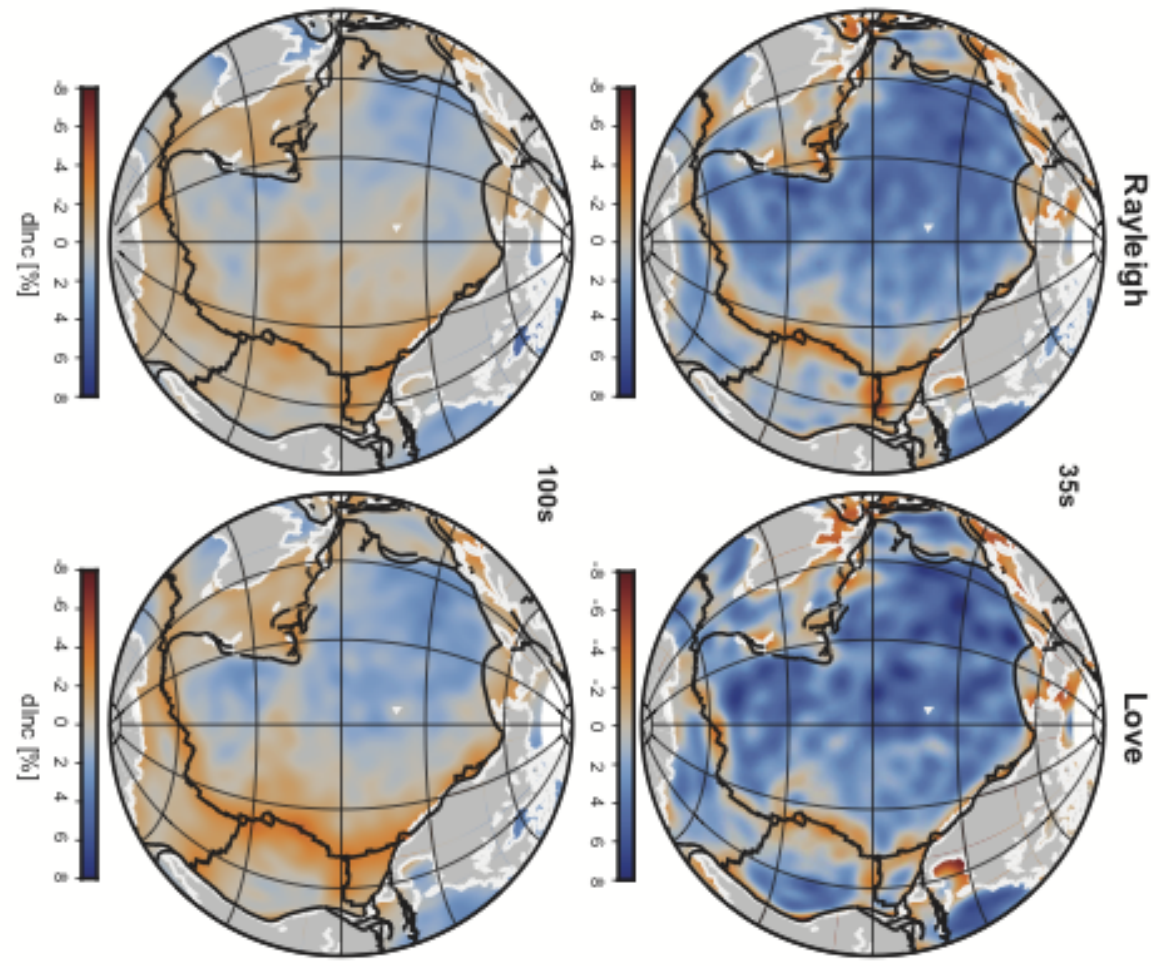

品

Figure 1. Love and Rayleigh wave phase velocity maps at 35s and 100s from the Visser et al. (2008a) dataset (left) and the Ekström (2011) dataset (right). Relative phase velocity perturbations are with respect to PREM (Dziewonski and Anderson, 1981). 

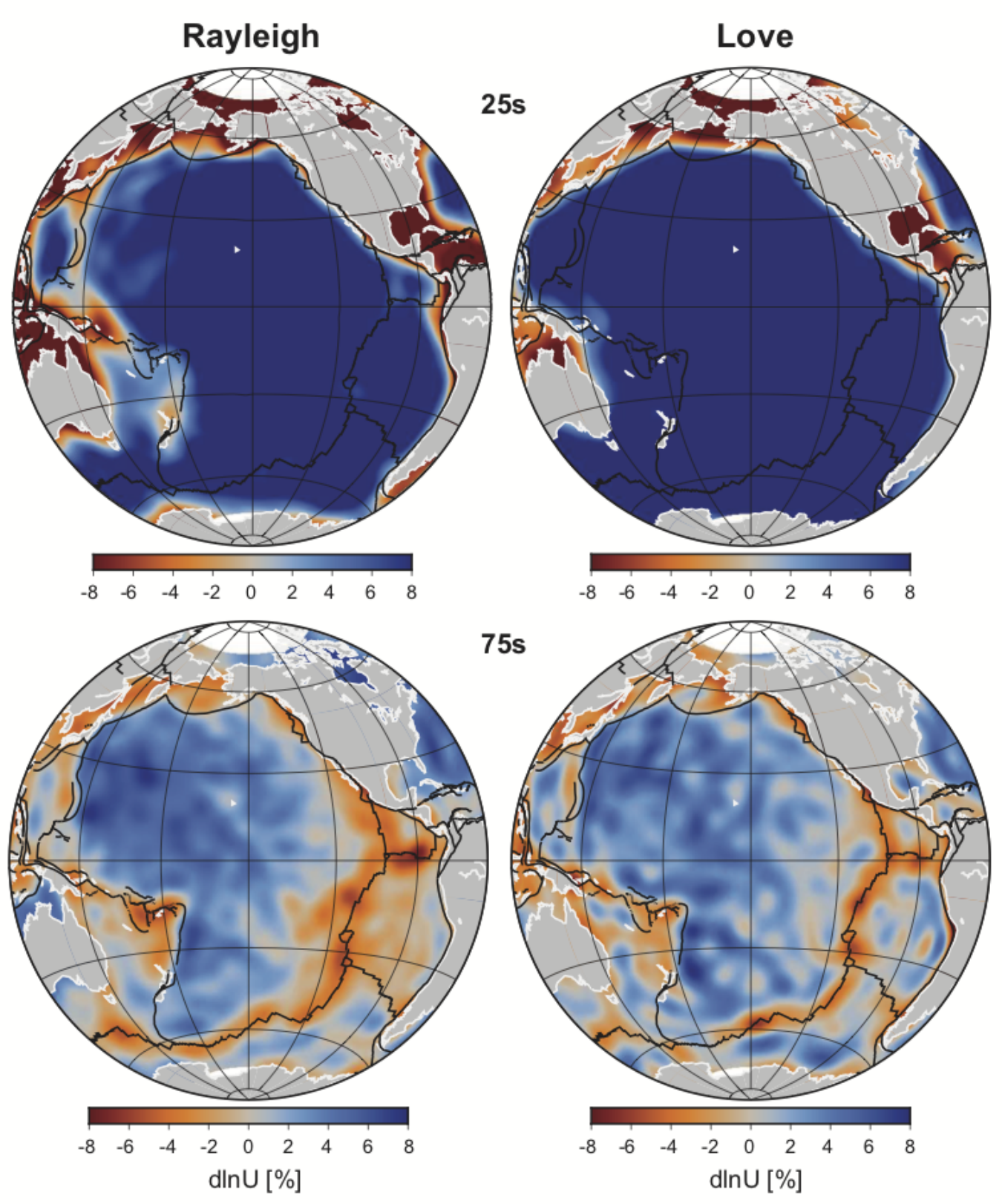

Figure 2. Love and Rayleigh wave group velocity maps at 25s and 75s from Ekström (2011). Relative group velocity perturbations are represented with respect to PREM (Dziewonski and Anderson, 1981). 
32 Beghein, C., Xing, Z. and Goes, S.
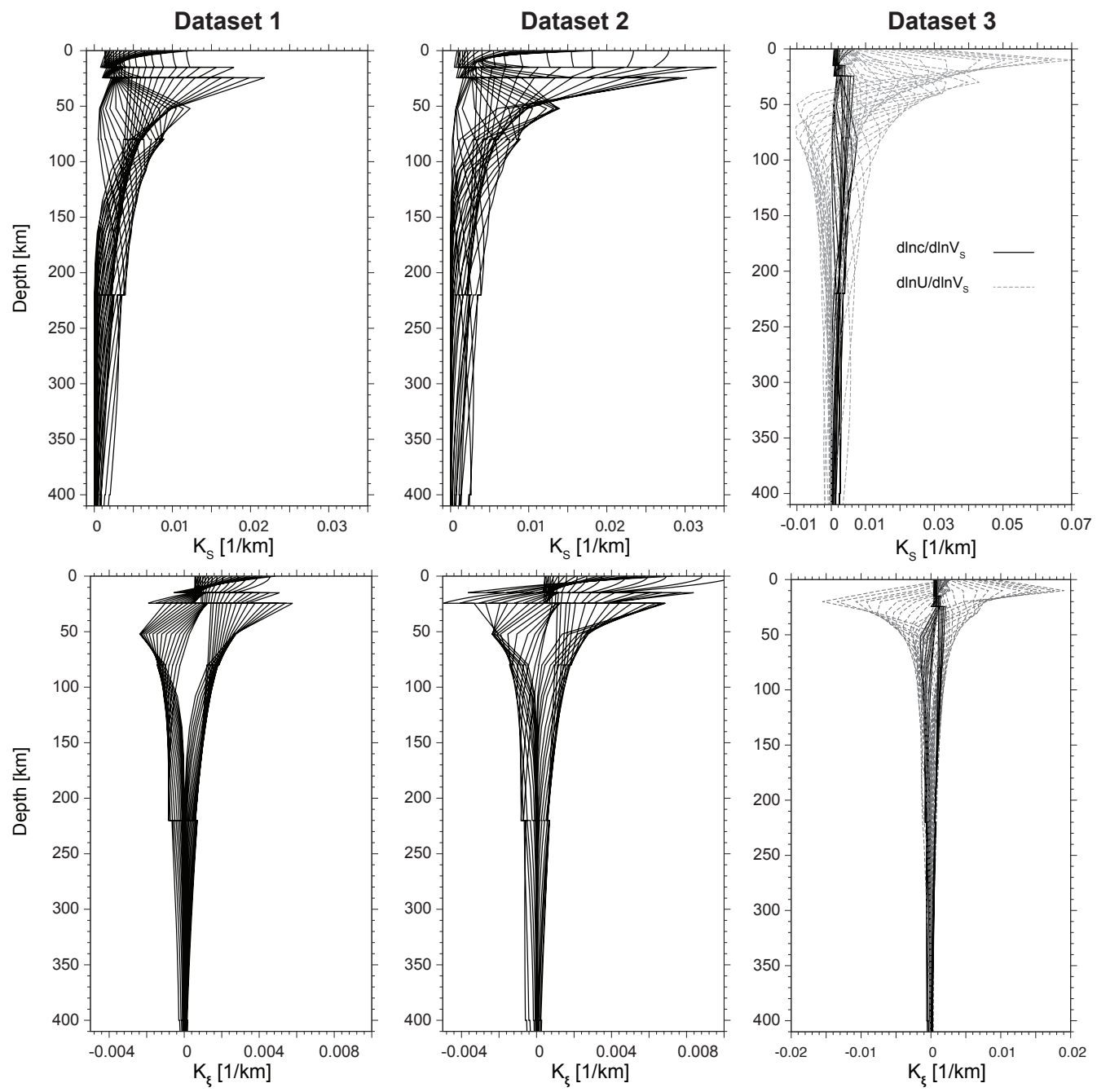

Figure 3. Sensitivity kernels for relative perturbations $d V_{S} / V_{S}$ in shear-wave velocities (top) and $d \xi / \xi$ radial anisotropy (bottom) for the three datasets described in section 2.1. The periods at which the phase velocity kernels were calculated range from $35 \mathrm{~s}$ and $175 \mathrm{~s}$ for dataset 1 and from $25 \mathrm{~s}$ and $250 \mathrm{~s}$ for dataset 2 . For dataset 3 , the periods of the phase velocity kernels range from 60 s to 250 s and from $25 \mathrm{~s}$ to 250 s for the group velocity kernels. 

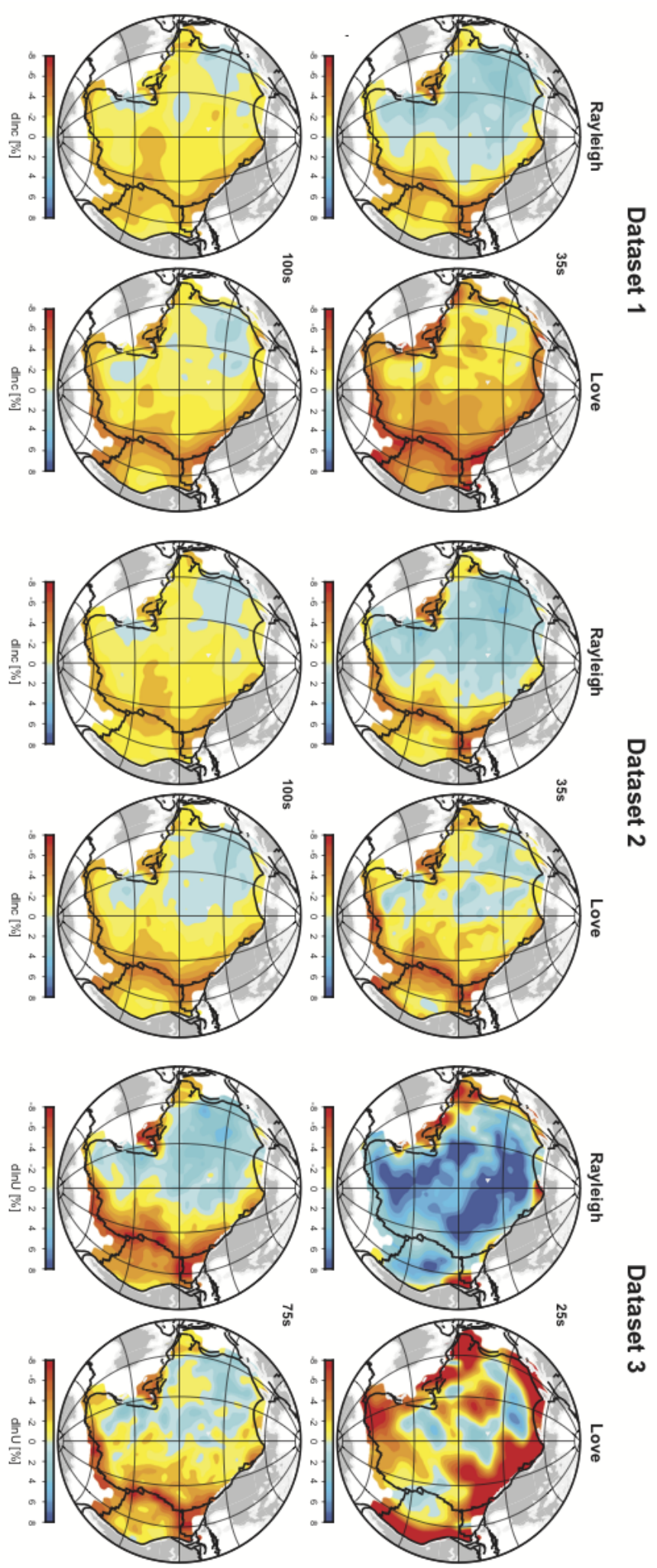

Figure 4. Examples of phase and group velocity maps corrected for the crust using CRUST1.0 (Laske et al., 2013) for each dataset used in this study. Perturbations in phase and group velocities are with respect to PREM (Dziewonski and Anderson, 1981). 
34 Beghein, C., Xing, Z. and Goes, S.
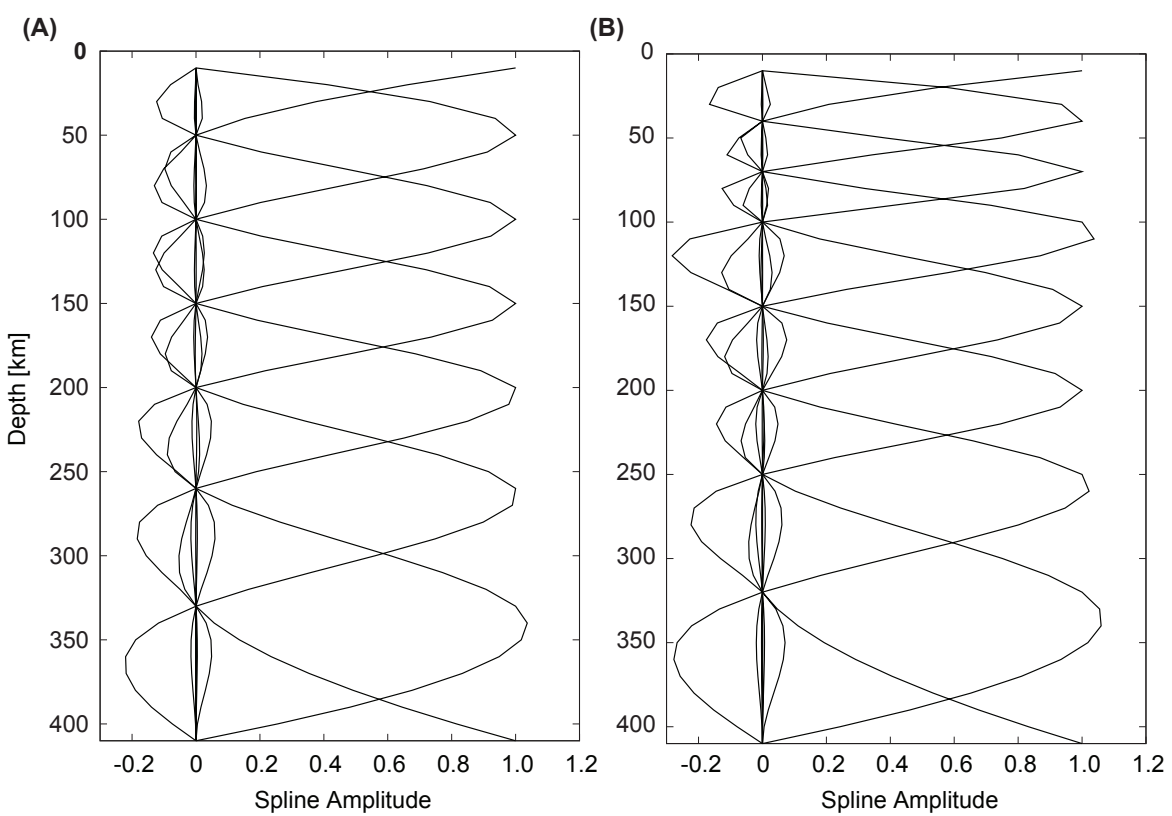

Figure 5. Cubic spline functions employed to parametrize vertically the model parameters in the inversions of dataset 1 and dataset 2 (left) and of dataset 3 (right). 

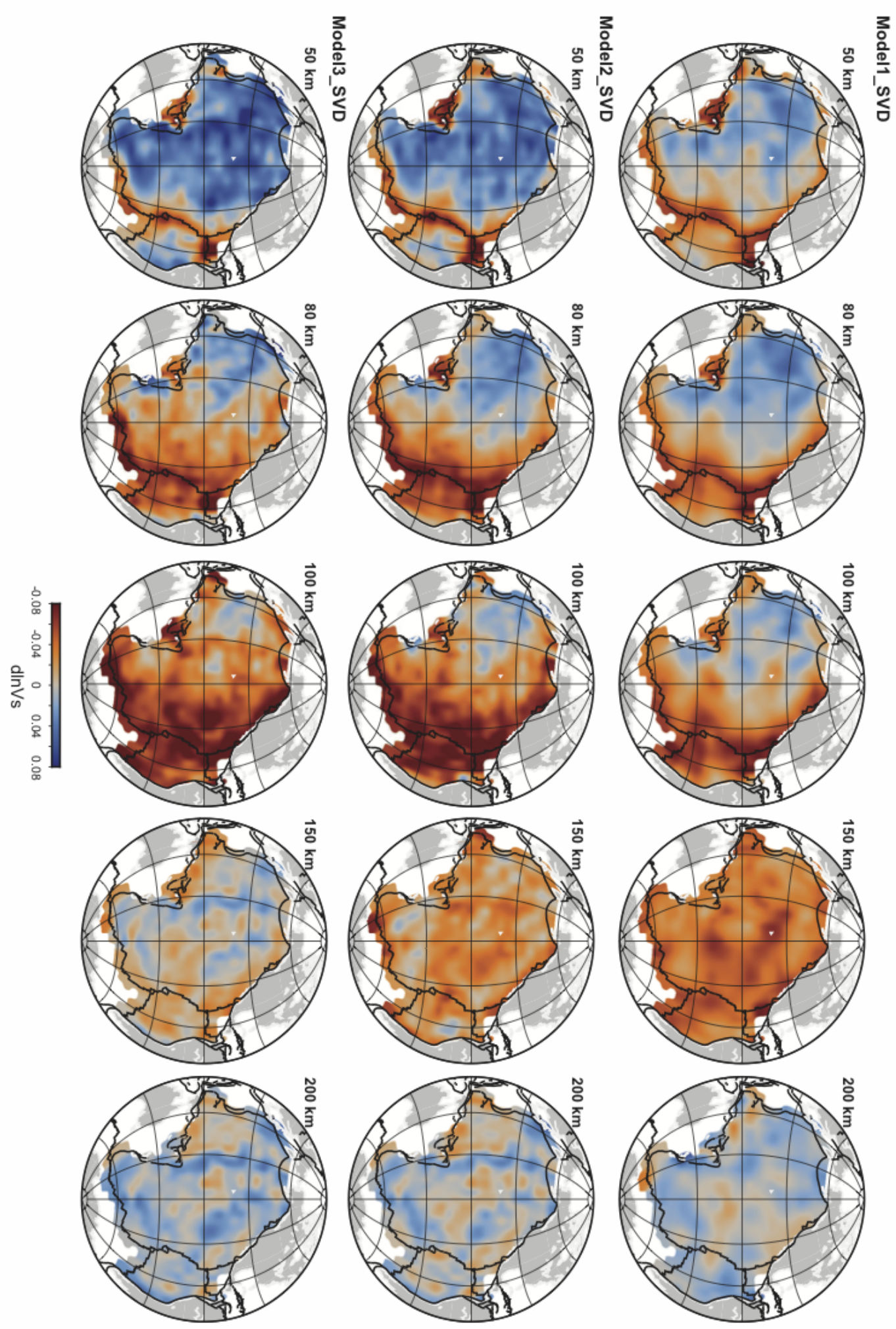

Figure 6. Maps of relative shear-wave velocity perturbations at different depths in the uppermost mantle. They were obtained from the regularized inversion of dataset 1 (top), dataset 2 (middle), and dataset 3 (bottom). Perturbations in $V_{S}$ are with respect to CRUST1.0 (Laske et al., 2013) superimposed onto anisotropic PREM (Dziewonski and Anderson, 1981). 
Beghein, C., Xing, Z. and Goes, S.
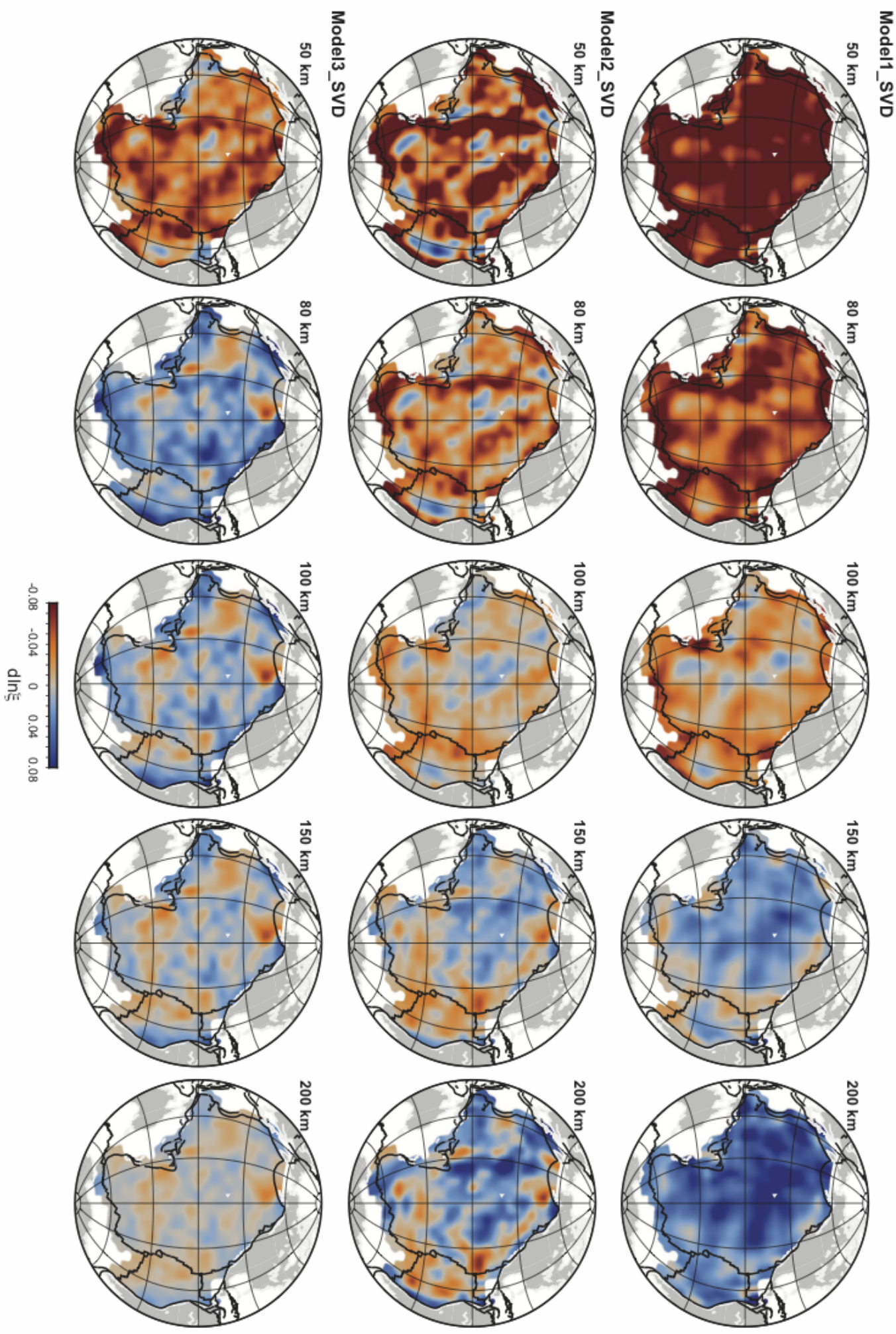

Figure 7. Maps of relative perturbations in shear-wave radial anisotropy at different depths in the uppermost mantle. They were obtained from the regularized inversion of dataset 1 (top) and dataset 2 (middle), and dataset 3 (bottom). Perturbations in $\xi$ are with respect to CRUST1.0 (Laske et al., 2013) superimposed onto anisotropic PREM (Dziewonski and Anderson, 1981). 
Model1_NA
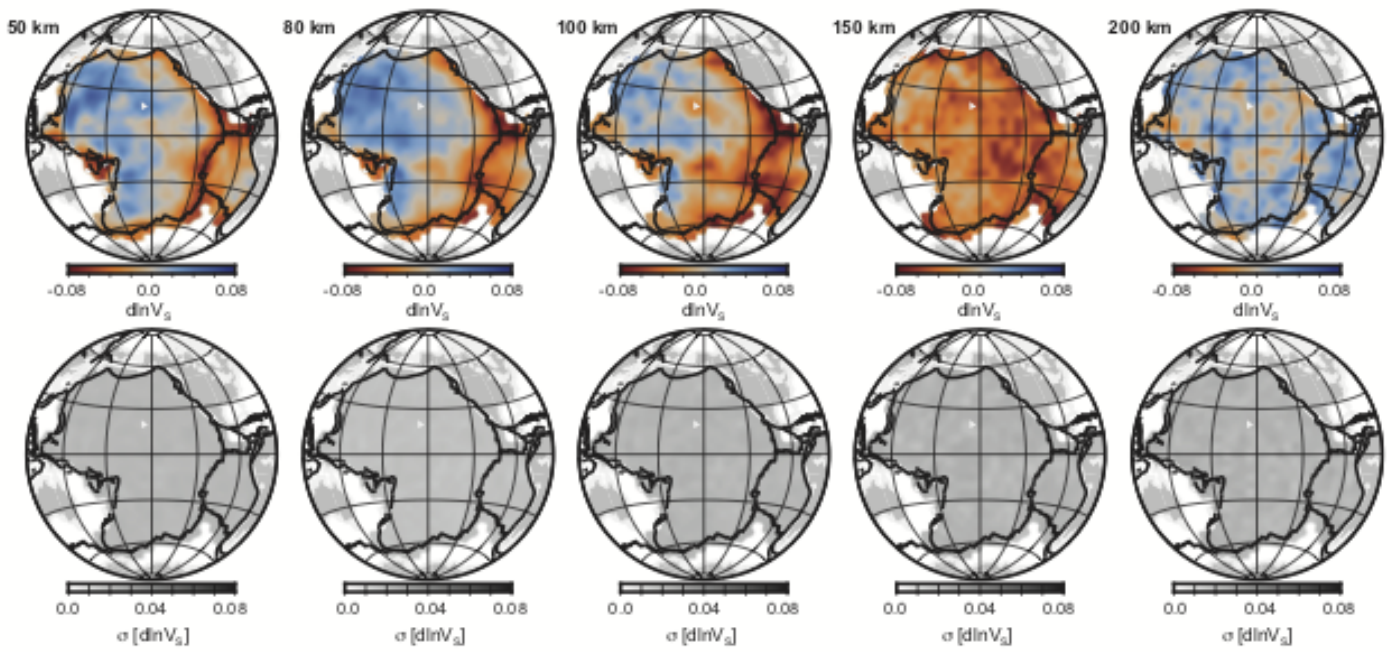

Model2_NA
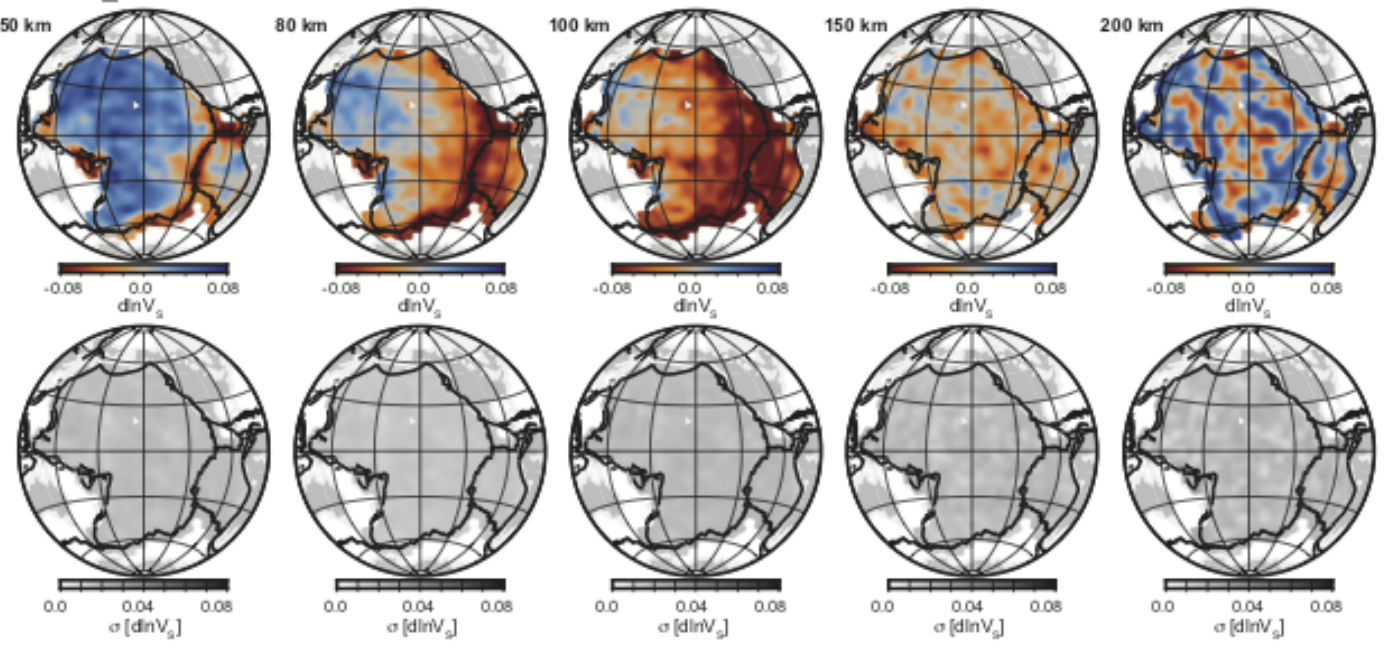

Model3_NA
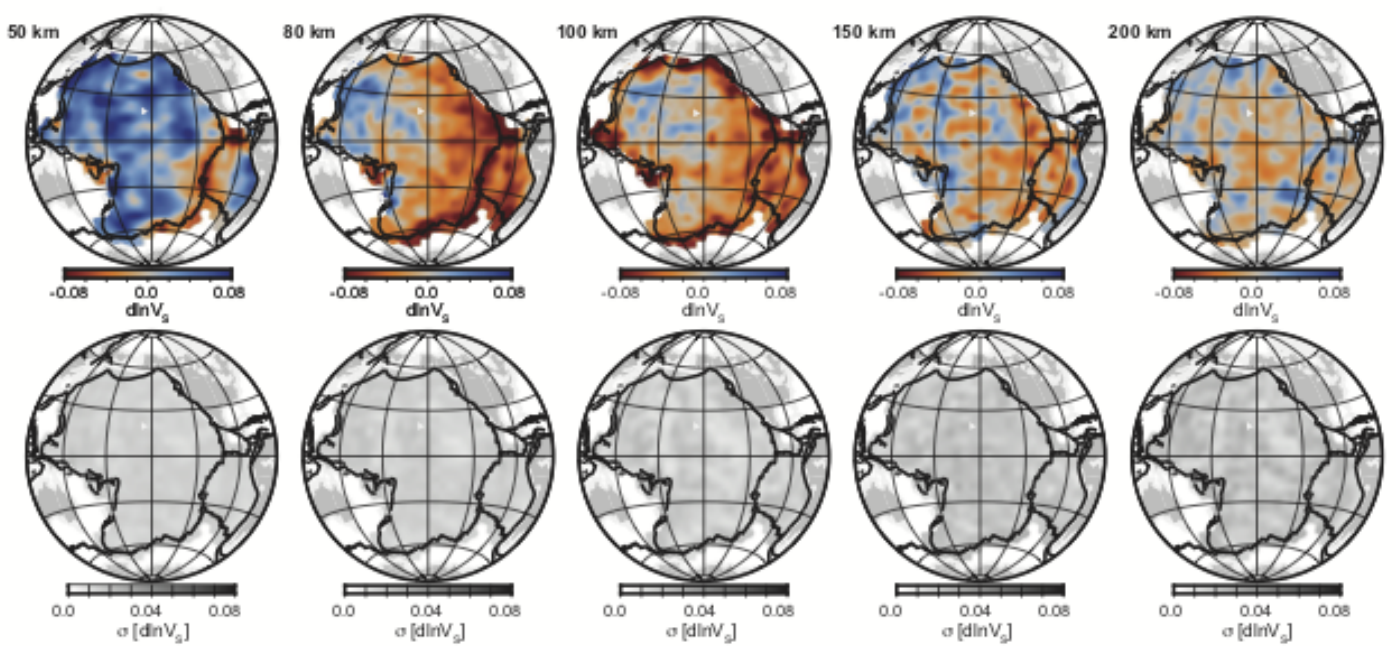

Figure 8. Mean velocity models and standard deviation obtained by applying the NA to dataset 1 (top) and dataset 2 (middle), and dataset 3 (bottom). 


\section{Model1_NA}
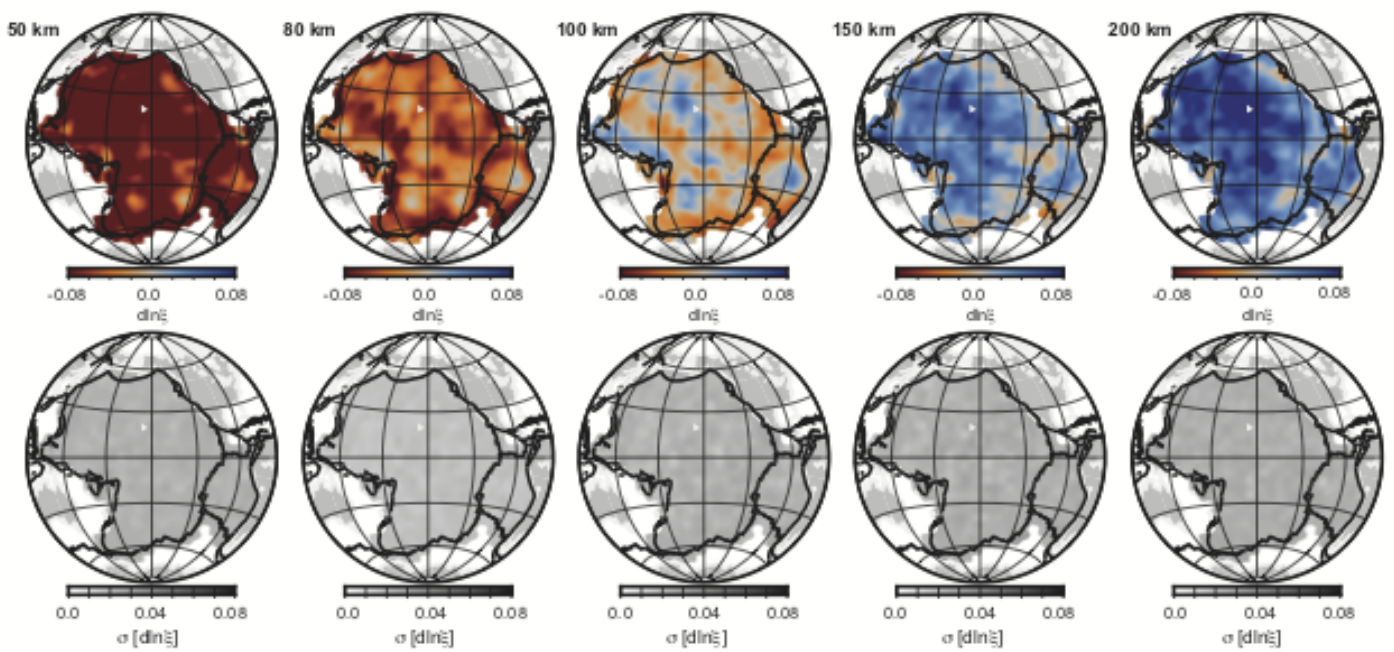

Model2_NA
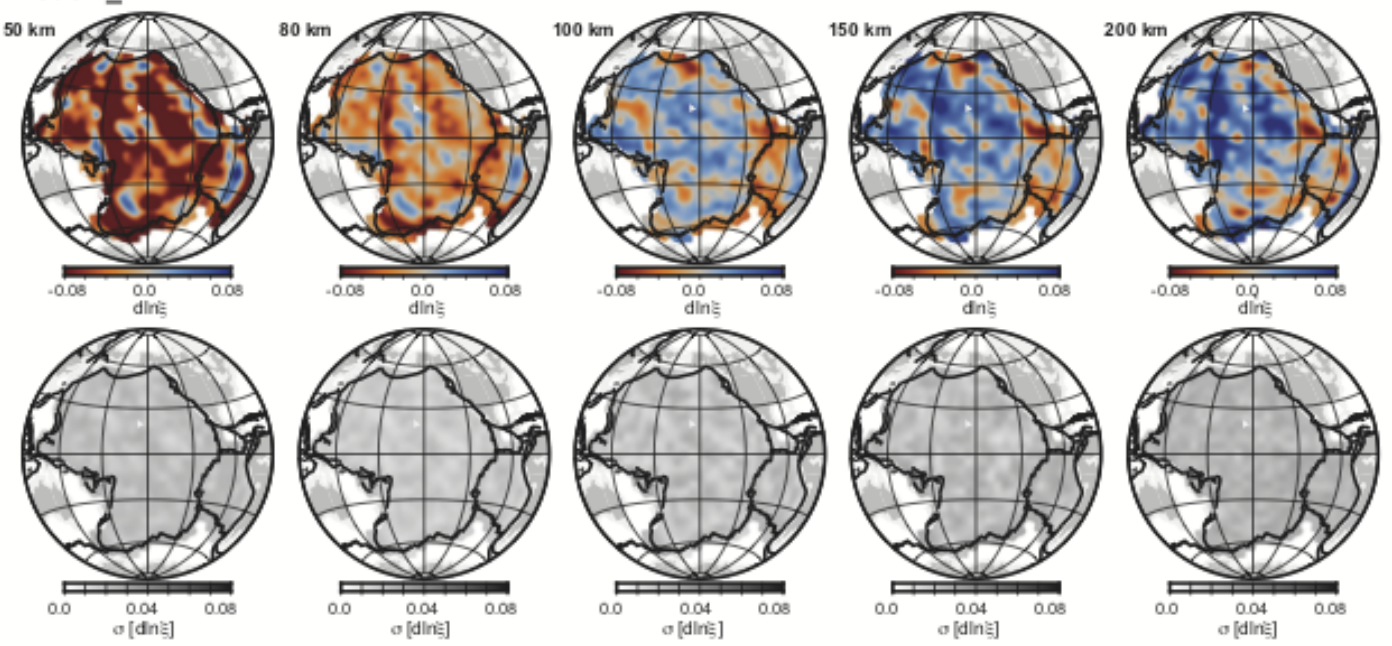

Model3_NA
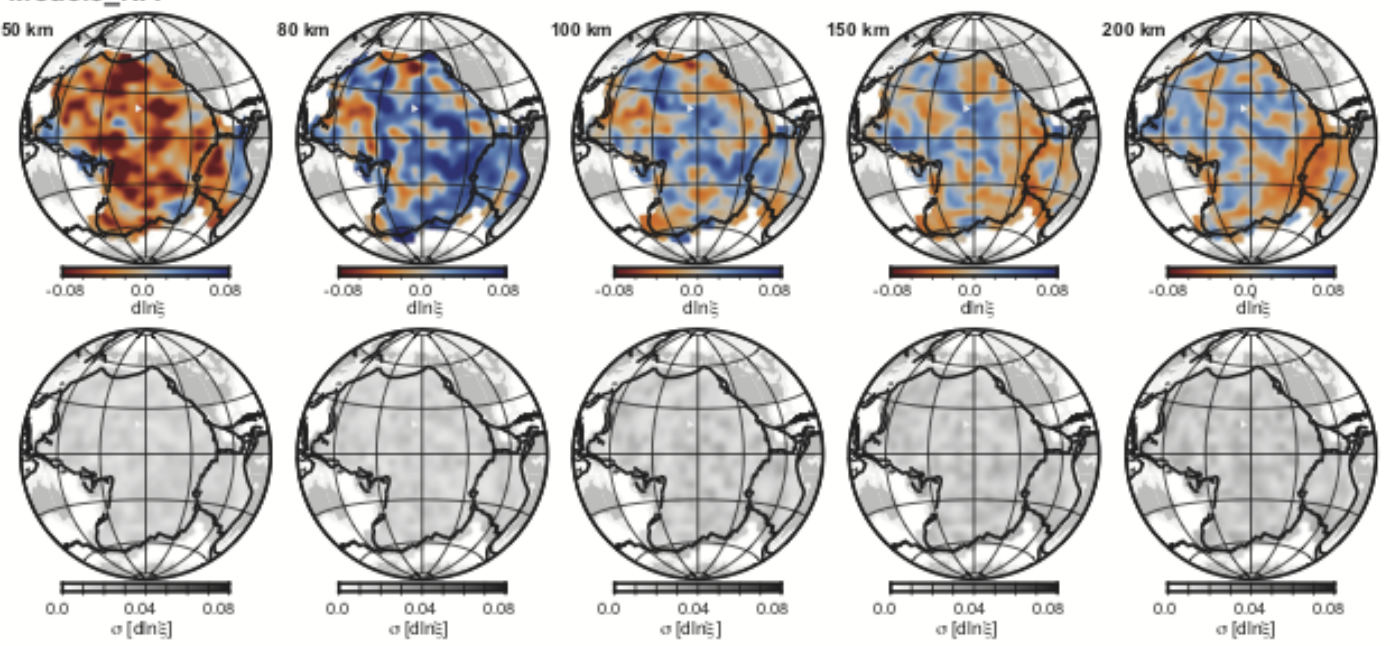

Figure 9. Mean radial anisotropy models and standard deviation obtained by applying the NA to dataset 1 (top) and dataset 2 (middle), and dataset 3 (bottom). 


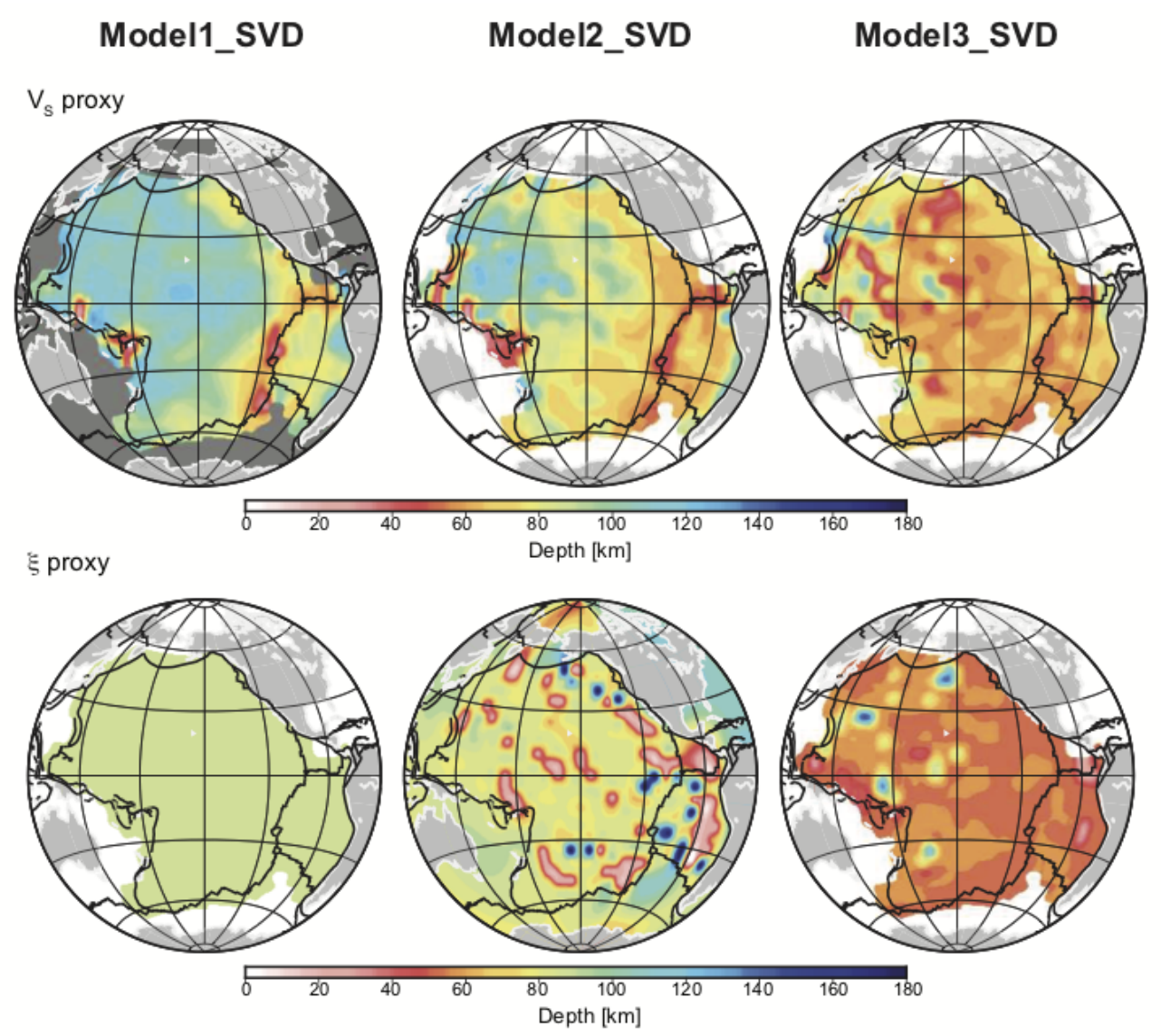

Figure 10. Maps of LAB depth calculated using the depth of the middle of the LVZ (top) and the depth of the largest positive radial anisotropy gradient (bottom) and the models obtained by regularized inversions of dataset 1 (left), dataset 2 (middle), and dataset 3 (right). 

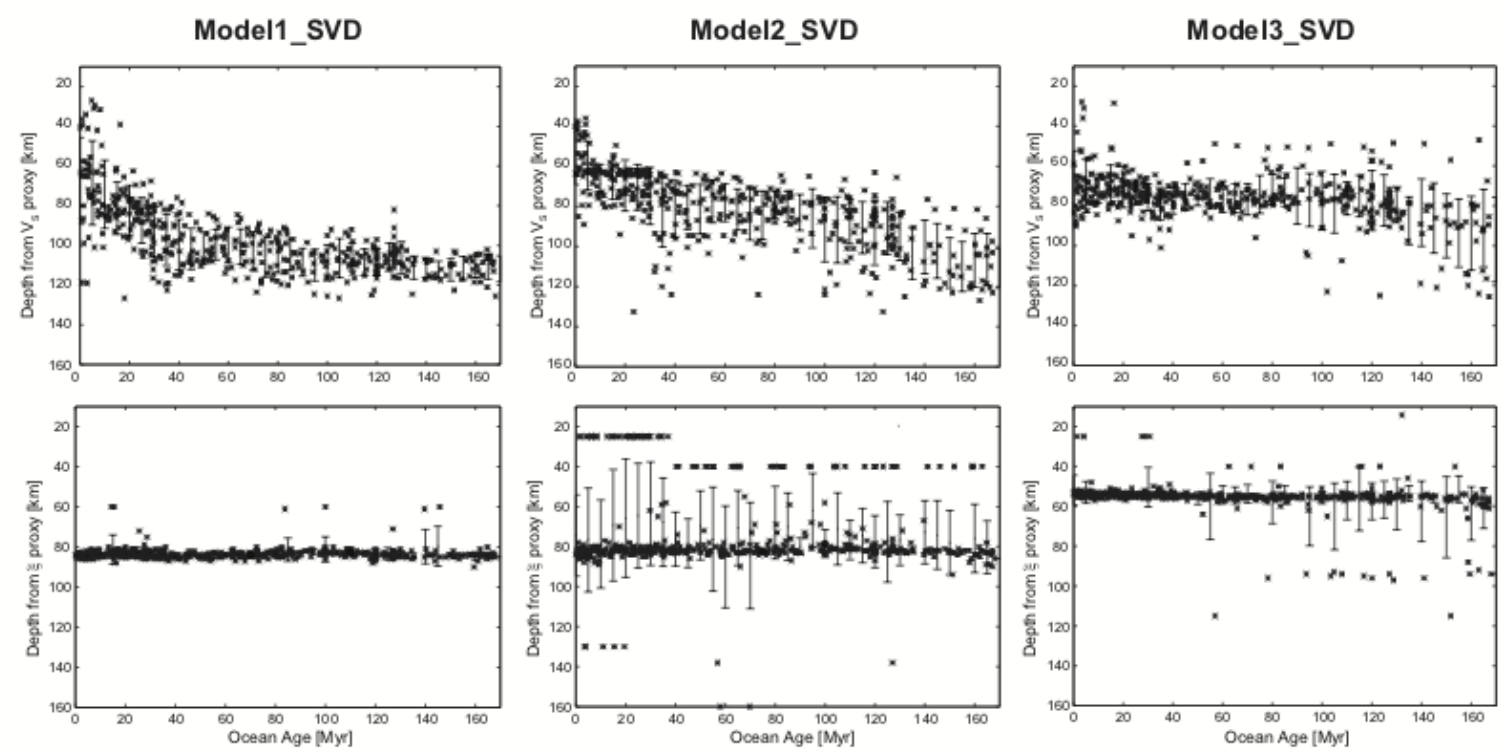

Figure 11. Age dependence of the LAB depth calculated using the depth of the middle of the LVZ (top) and the depth of the largest positive radial anisotropy gradient (bottom) from the models obtained by regularized inversions of dataset 1 (left), dataset 2 (middle), and dataset 3 (right). The vertical error bars show one standard deviation representing variations within the model binned every $5 \mathrm{My}$. 


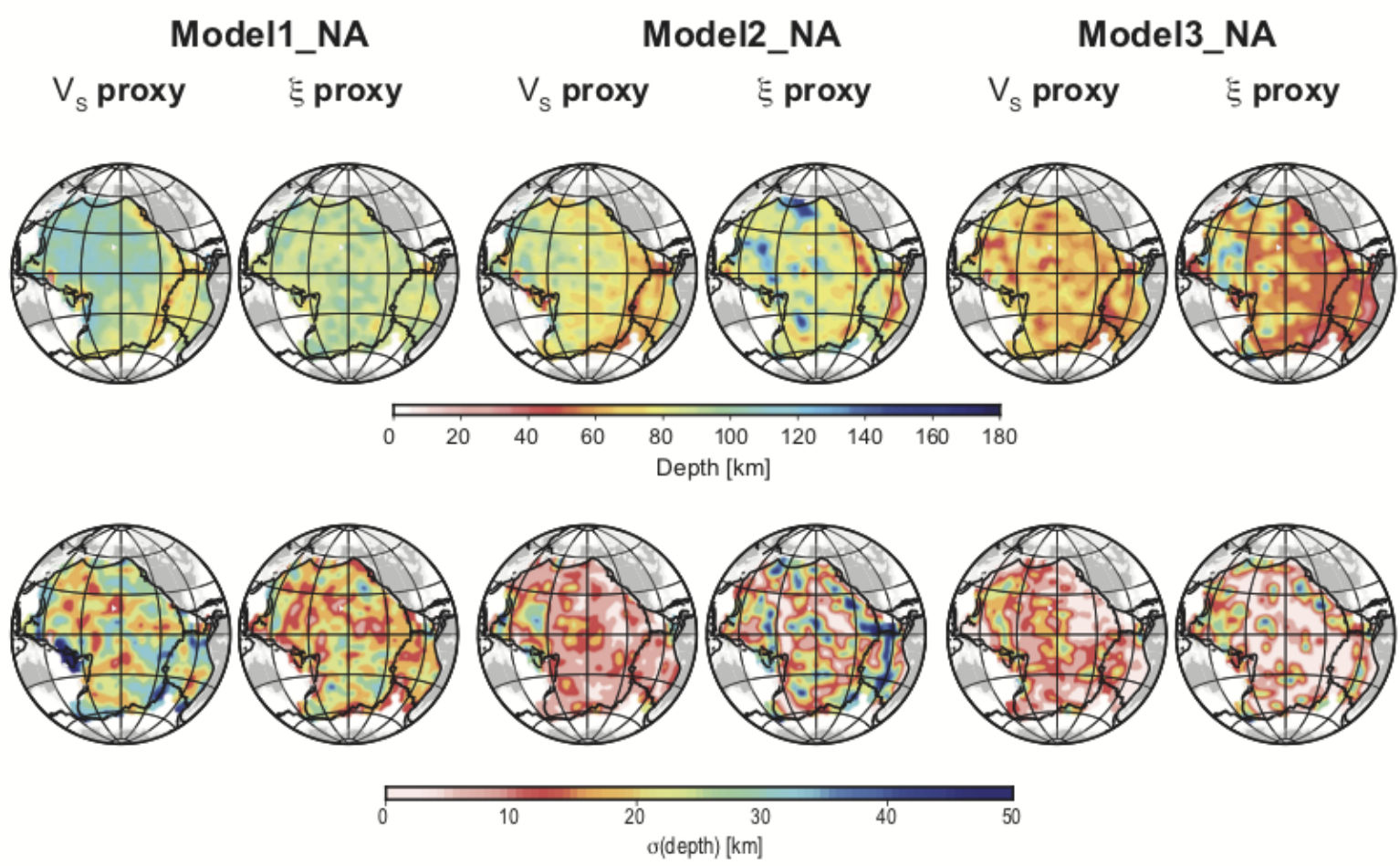

Figure 12. Mean LAB depth (top) and standard deviation (bottom) calculated using the depth of the middle of the LVZ and the depth of the largest positive radial anisotropy gradient and the models obtained by applying NA to dataset 1 (left), dataset 2 (middle), and dataset 3 (right) 

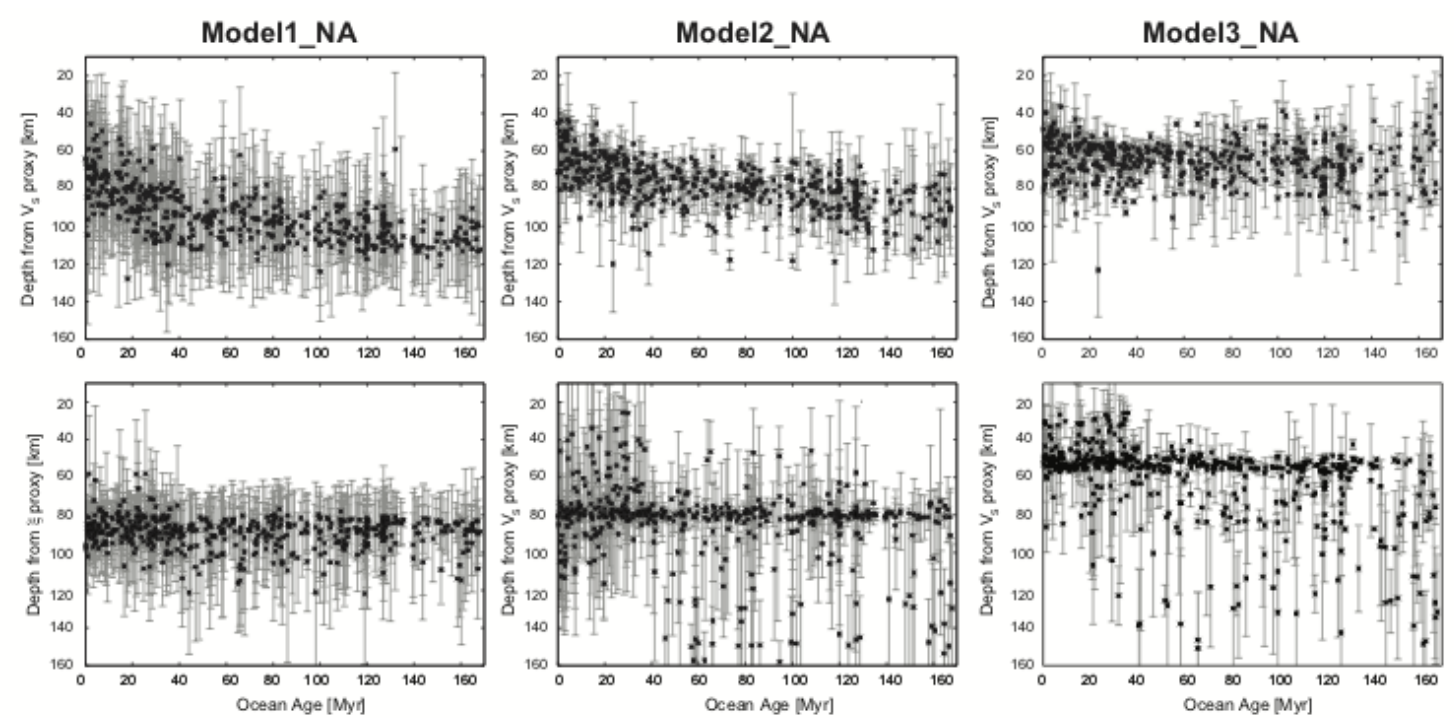

Figure 13. Age dependence of the LAB depth calculated using the depth of the middle of the LVZ (top) and the depth of the largest positive radial anisotropy gradient (bottom) from the models obtained by applying the NA to the three datasets. The mean of the LAB depth distribution calculated at every grid cell is shown with one standard deviation. 

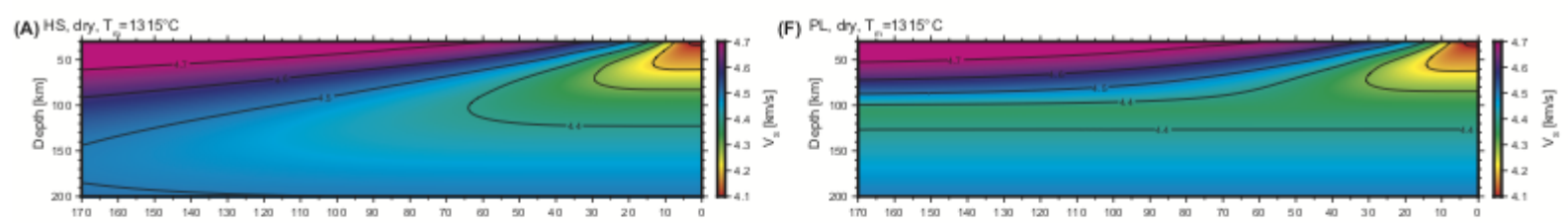

(B) HS, damp, $T_{n}=1315{ }^{\circ} \mathrm{C}$

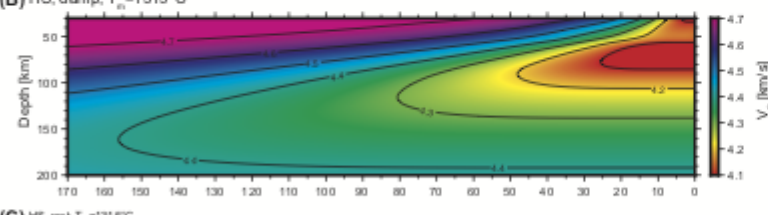

(G) PL, damp, $T_{\mathrm{g}}=1315^{\circ} \mathrm{C}$

(C) $15,0 \mathrm{det} T_{z}=1315 \mathrm{C}$
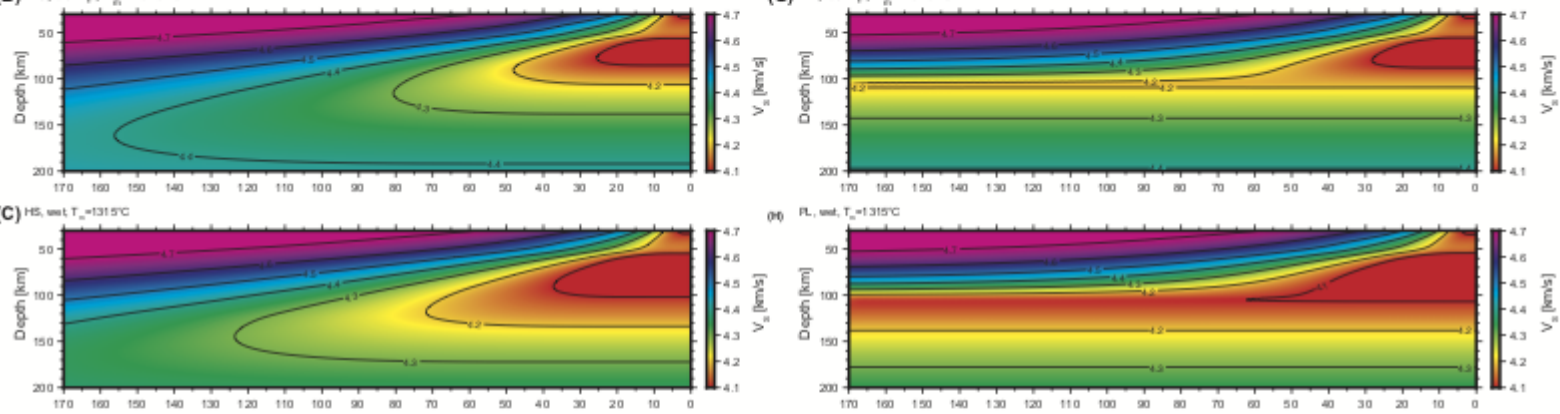
(10) R., $2, T_{n}=13150$

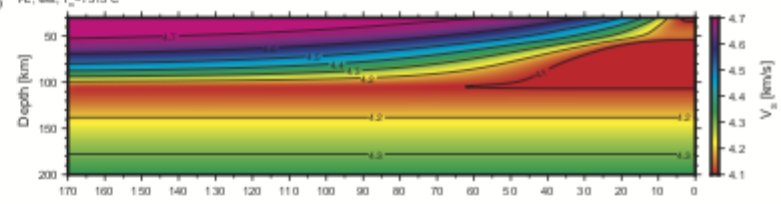

(D) HS, dry, $\mathrm{T}_{\mathrm{e}}=1415^{\circ} \mathrm{C}$

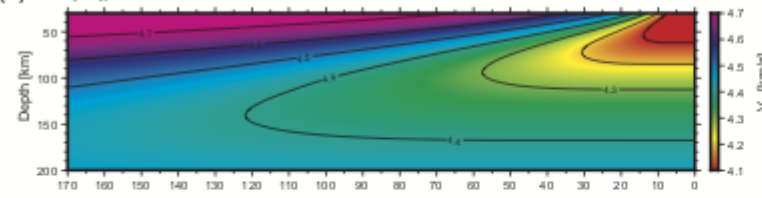

(I) PL, dy, $\mathrm{T}_{\mathrm{s}}=1415^{\circ} \mathrm{C}$ (E) HS, damp, $T_{n}=1415^{\circ} \mathrm{C}$
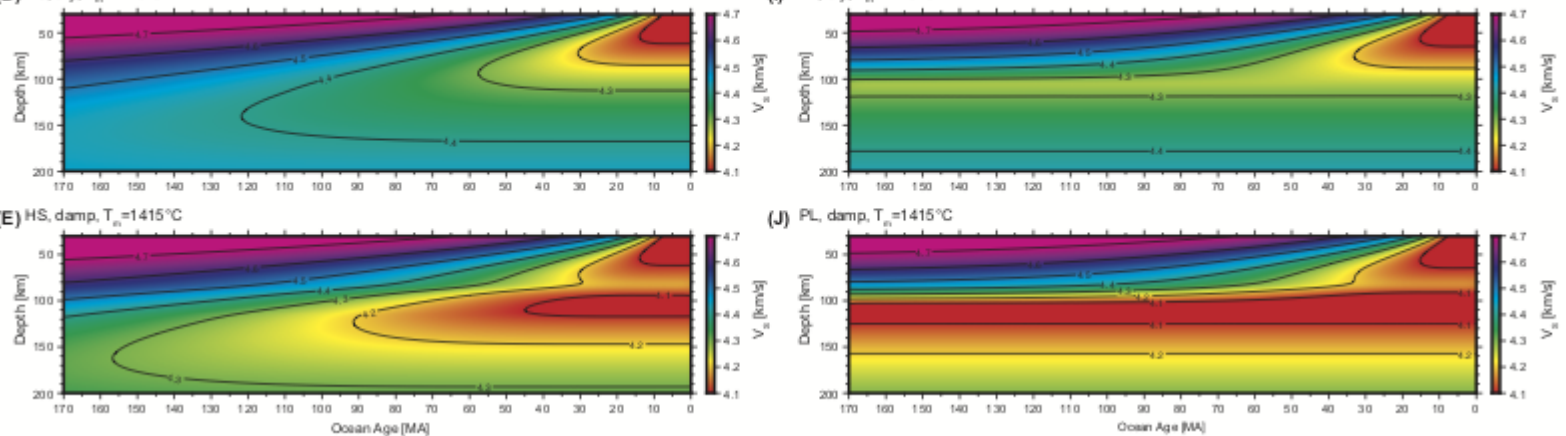
(J) PL, damp, $T_{e}=1415^{\circ} \mathrm{C}$

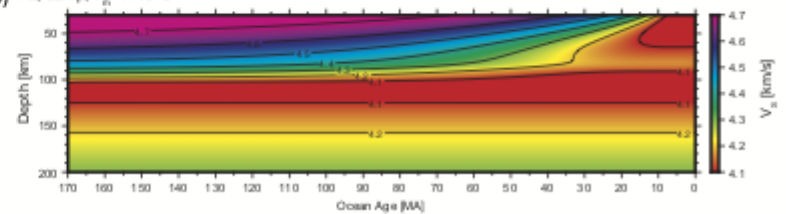

Figure 14. Synthetic velocity models from Goes et al. (2012) using empirical attenuation model $Q_{g}$. We determined $V_{S}$ in five cases for each cooling model: a dry mantle with potential temperature $T_{m}=1315^{\circ} \mathrm{C}((\mathrm{A})$ and (F)), a damp mantle with $T_{m}=1315^{\circ} \mathrm{C}\left((\mathrm{B})\right.$ and (G)), a wet mantle with $T_{m}=1315^{\circ} \mathrm{C}((\mathrm{C})$ and $(\mathrm{H}))$, a dry but hotter mantle with $T_{m}=1415^{\circ} \mathrm{C}\left((\mathrm{D})\right.$ and (I)), and a damp mantle with $T_{m}=1415^{\circ} \mathrm{C}((\mathrm{E})$ and $(\mathrm{J}))$. Other thermal parameters were chosen to match those of McKenzie et al. (2005). 
44 Beghein, C., Xing, Z. and Goes, S.
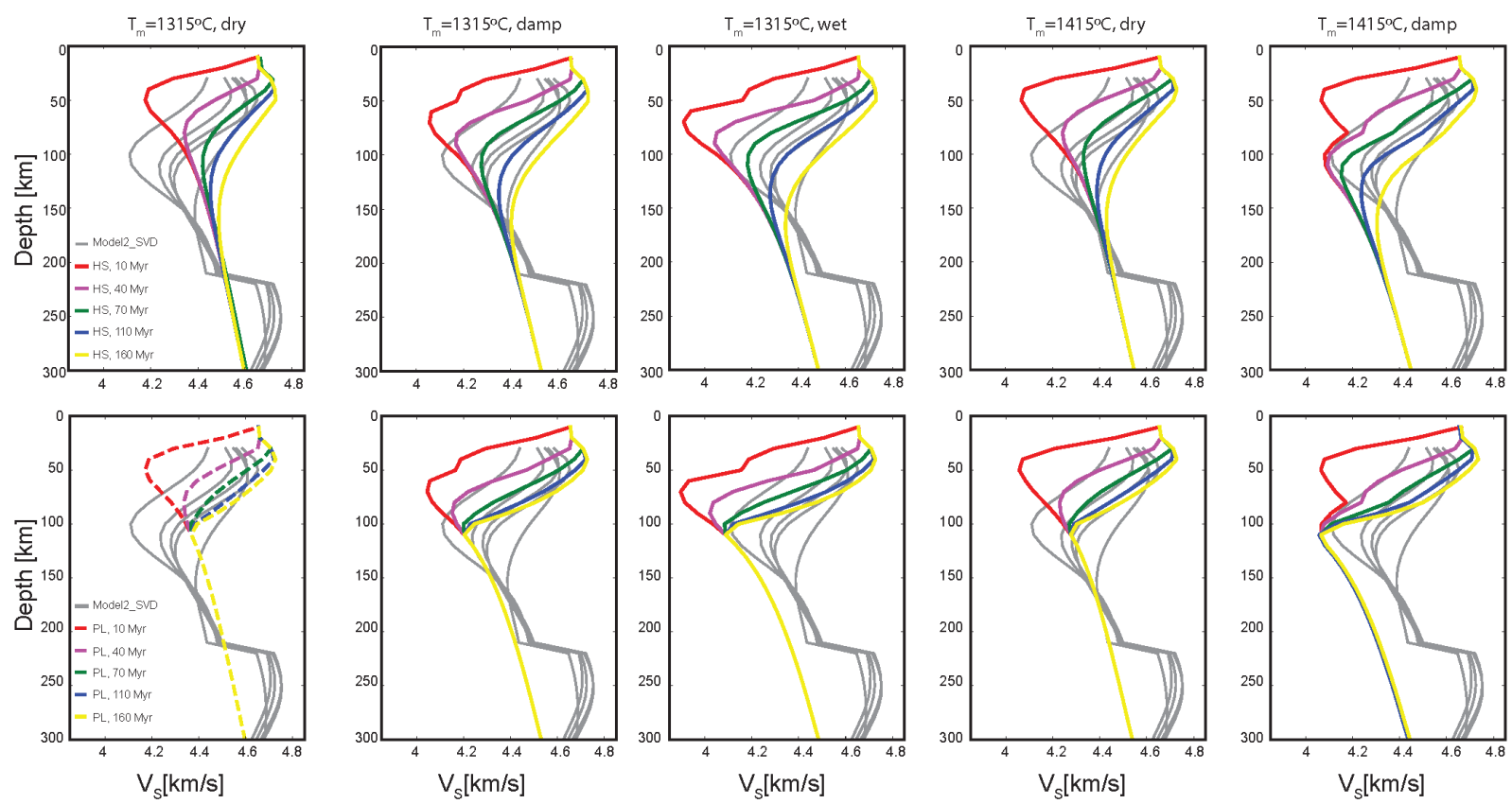

Figure 15. Comparison between velocity depth profiles obtained from the synthetic $V_{S}$ models described in Figure 14 for different ocean floor ages (colored curves) and from the $V_{S}$ model obtained by regularized inversion of dataset 2 (grey curves) plotted for the same age bins as the synthetic models. Synthetic models for HS cooling models are shown in the top panels and those for plate models are displayed in the bottom panels. 

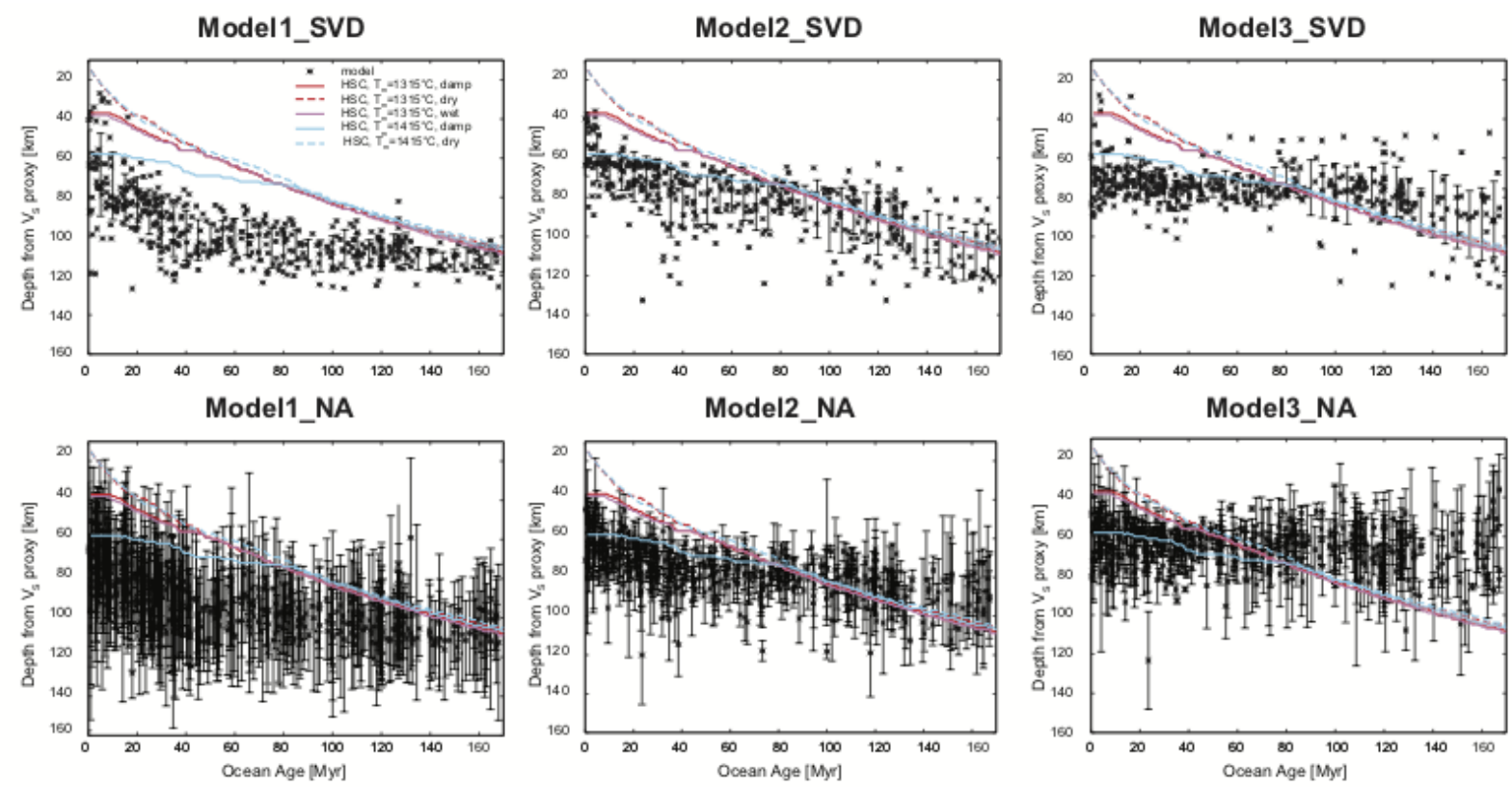

Figure 16. Comparison between LAB depth estimates from our $V_{S}$ tomographic models and from synthetic velocity models calculated as following Goes et al. (2012). The LAB depth proxy is calculated using the depth of the middle of the low velocity zone. The tomographic models used were obtained from dataset 1 (left), dataset 2 (middle), and dataset 3 (right). Models obtained by regularized inversion are displayed on top and models obtained using the NA are shown at the bottom. The vertical error bars are the same as in Figs. 11 and 13 The colored curves represent the LAB depth proxy calculated from the synthetic velocities shown in Figure 14 and determined for half-space cooling models. 


\section{Supplementary Material}

\section{Thermal Nature and Resolution of the Lithosphere-Asthenosphere Boundary Under the Pacific From Surface Waves}

Beghein, C., Xing, Z., and Goes, S. 

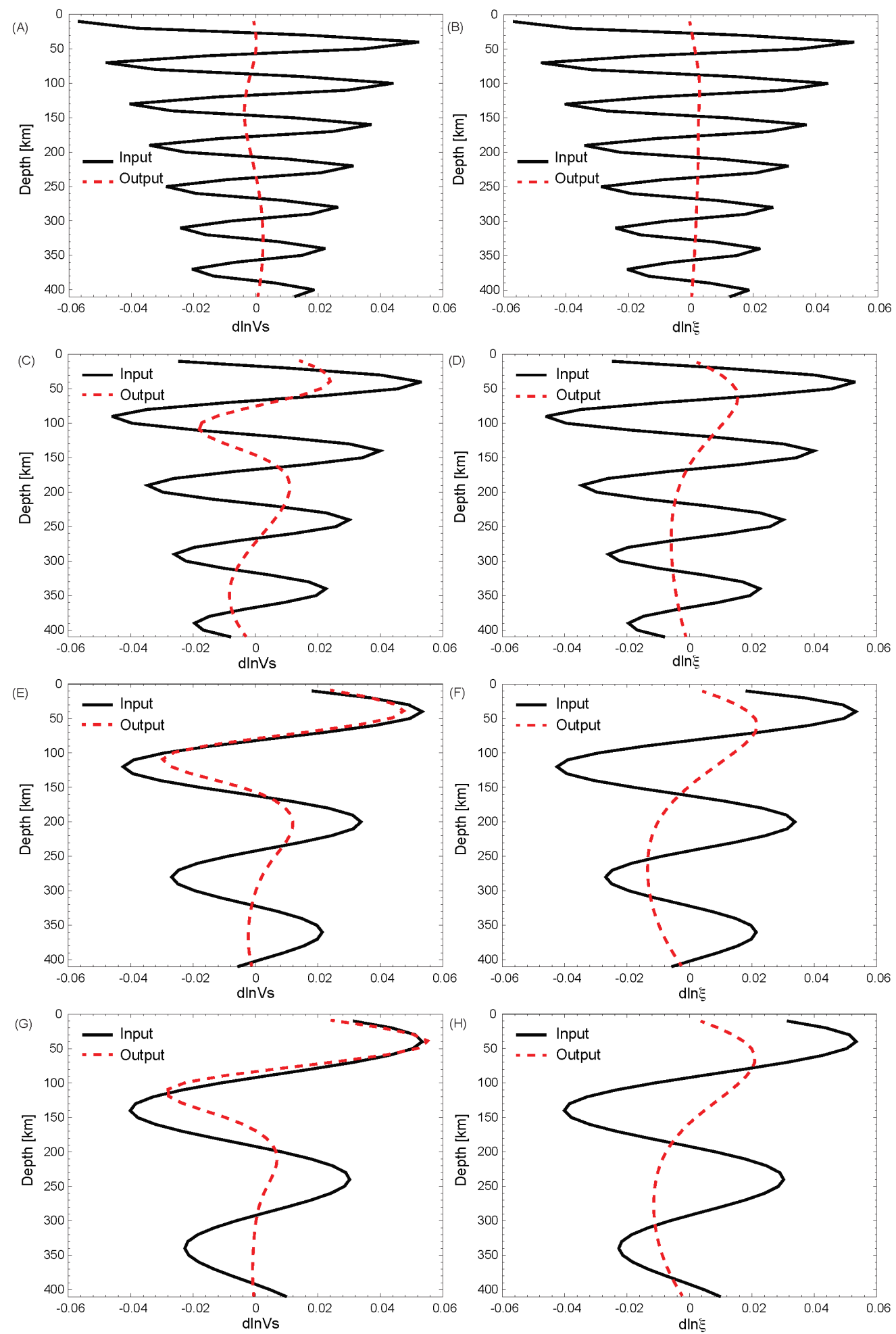

Figure S1: Synthetic tests using dataset 1 (Visser et al., 2008) for layer thicknesses of $30 \mathrm{~km}$ (A \& B), 50km (C \& D), 70km (E \& F), and 100km (G \& H). 

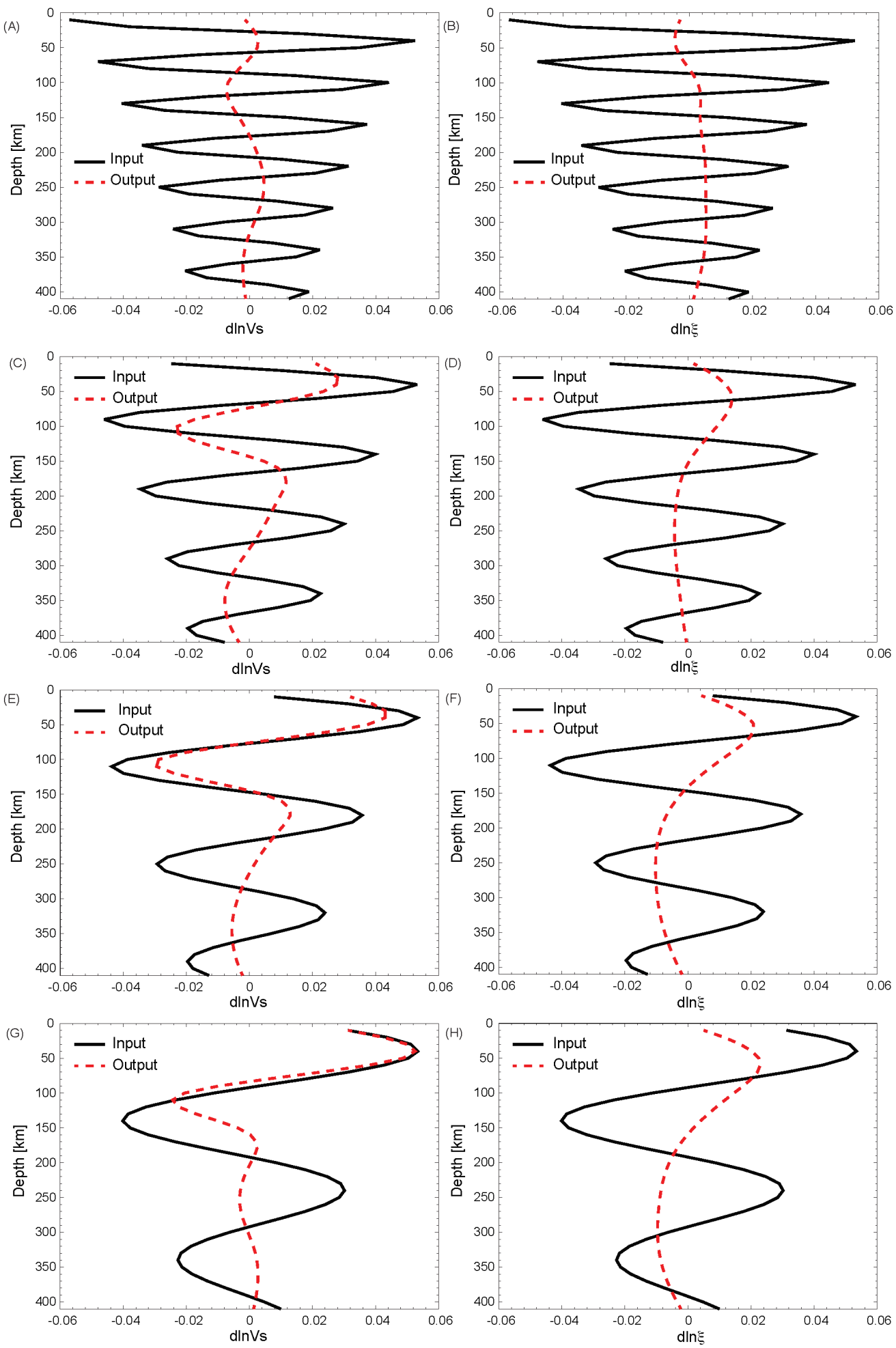

Figure S2: Synthetic tests using dataset 2 (Ekstrom et al., 2011) for layer thicknesses of $30 \mathrm{~km}(\mathrm{~A} \& \mathrm{~B}), 50 \mathrm{~km}(\mathrm{C} \& \mathrm{D}), 70 \mathrm{~km}(\mathrm{E} \& \mathrm{~F})$, and $100 \mathrm{~km}(\mathrm{G} \& \mathrm{H})$. 

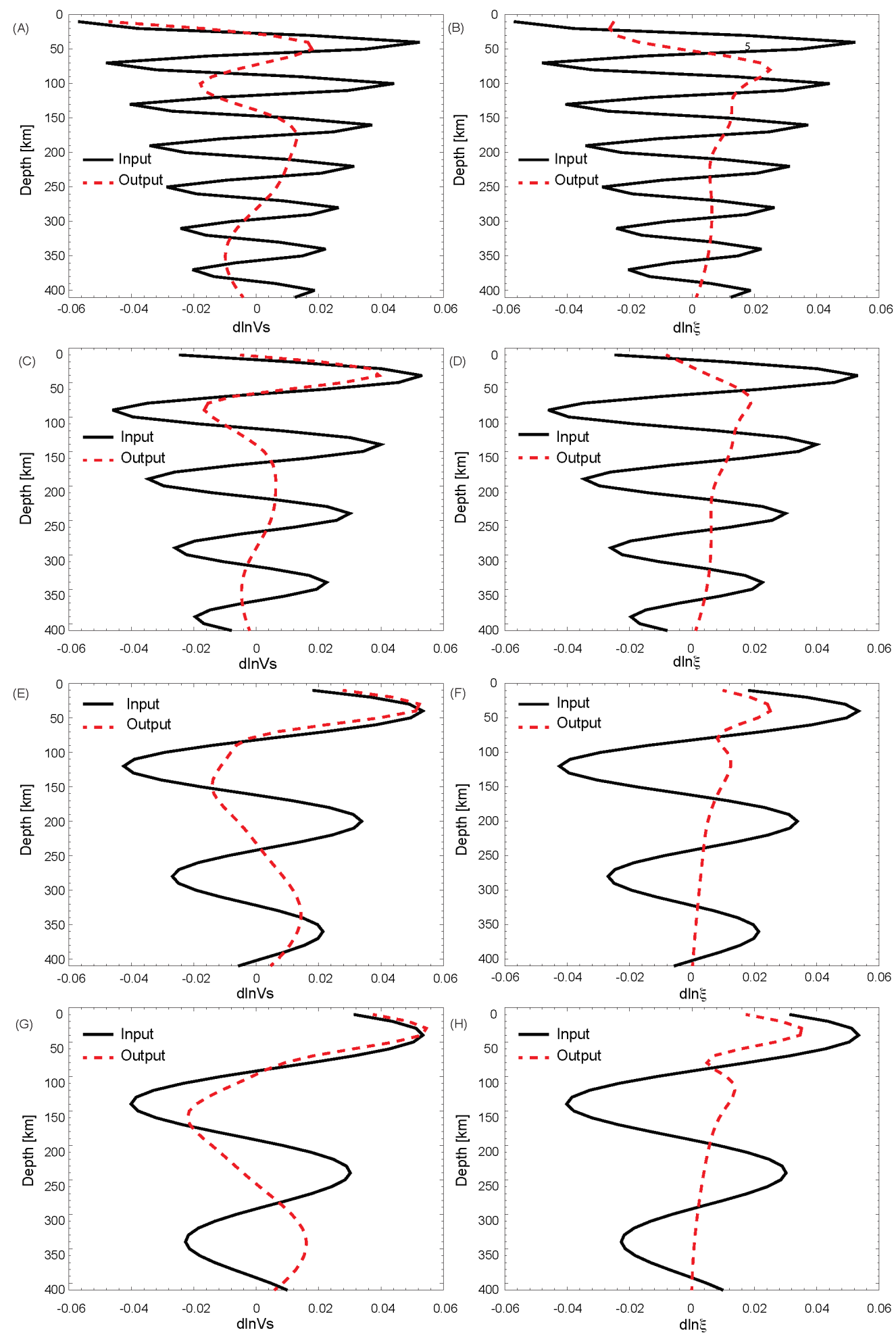

Figure S3: Synthetic tests using dataset 3 (Ekstrom et al., 2011) for layer thicknesses of $30 \mathrm{~km}(\mathrm{~A} \& \mathrm{~B}), 50 \mathrm{~km}(\mathrm{C} \& \mathrm{D}), 70 \mathrm{~km}(\mathrm{E} \& \mathrm{~F})$, and $100 \mathrm{~km}(\mathrm{G} \& \mathrm{H})$. 

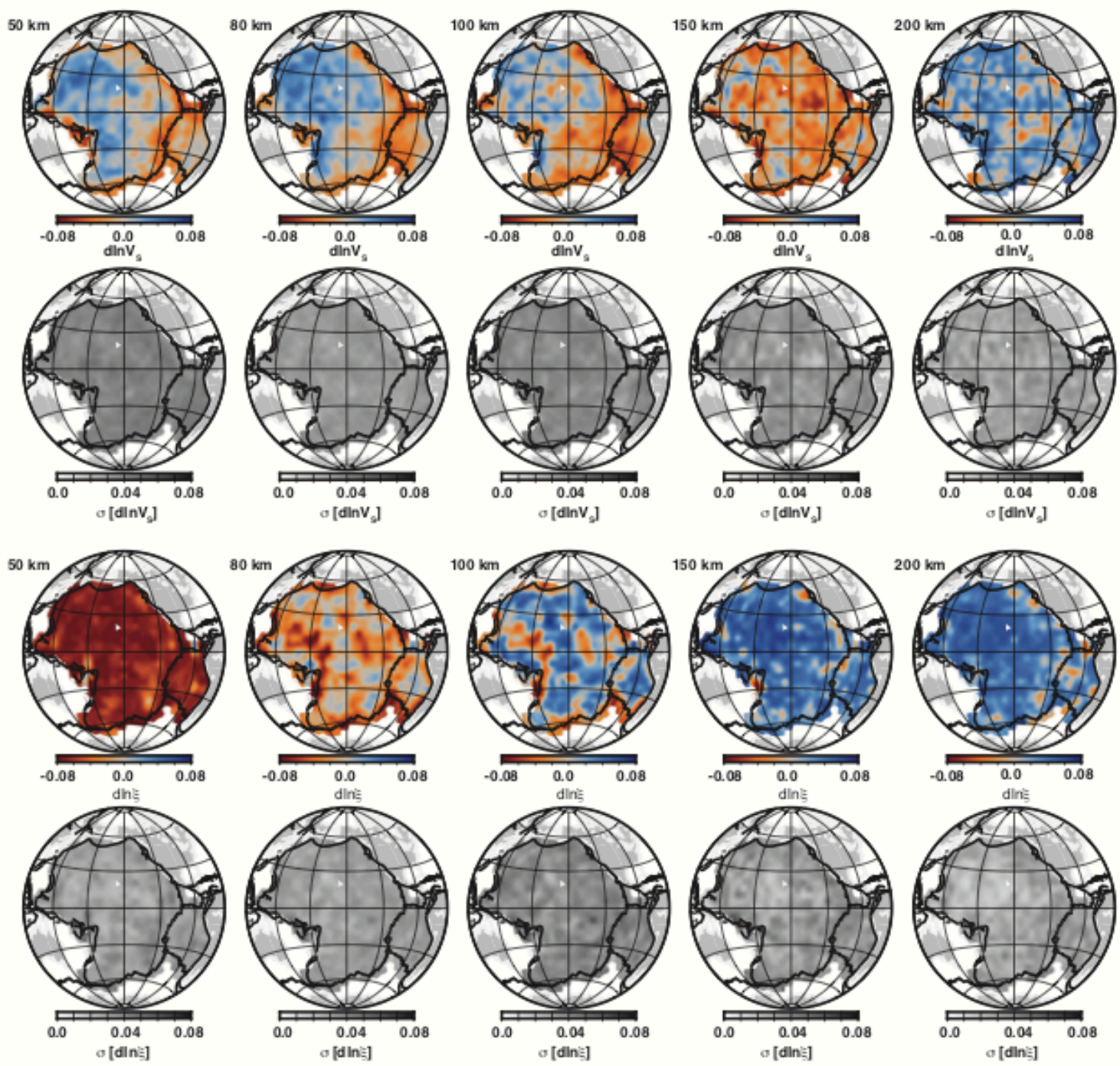

Figure S4: Mean relative velocity (top) and radial anisotropy (bottom) perturbations and standard deviations obtained with dataset 1 and NA with a model space search centered around PREM (Dziewonski and Anderson, 1981). 

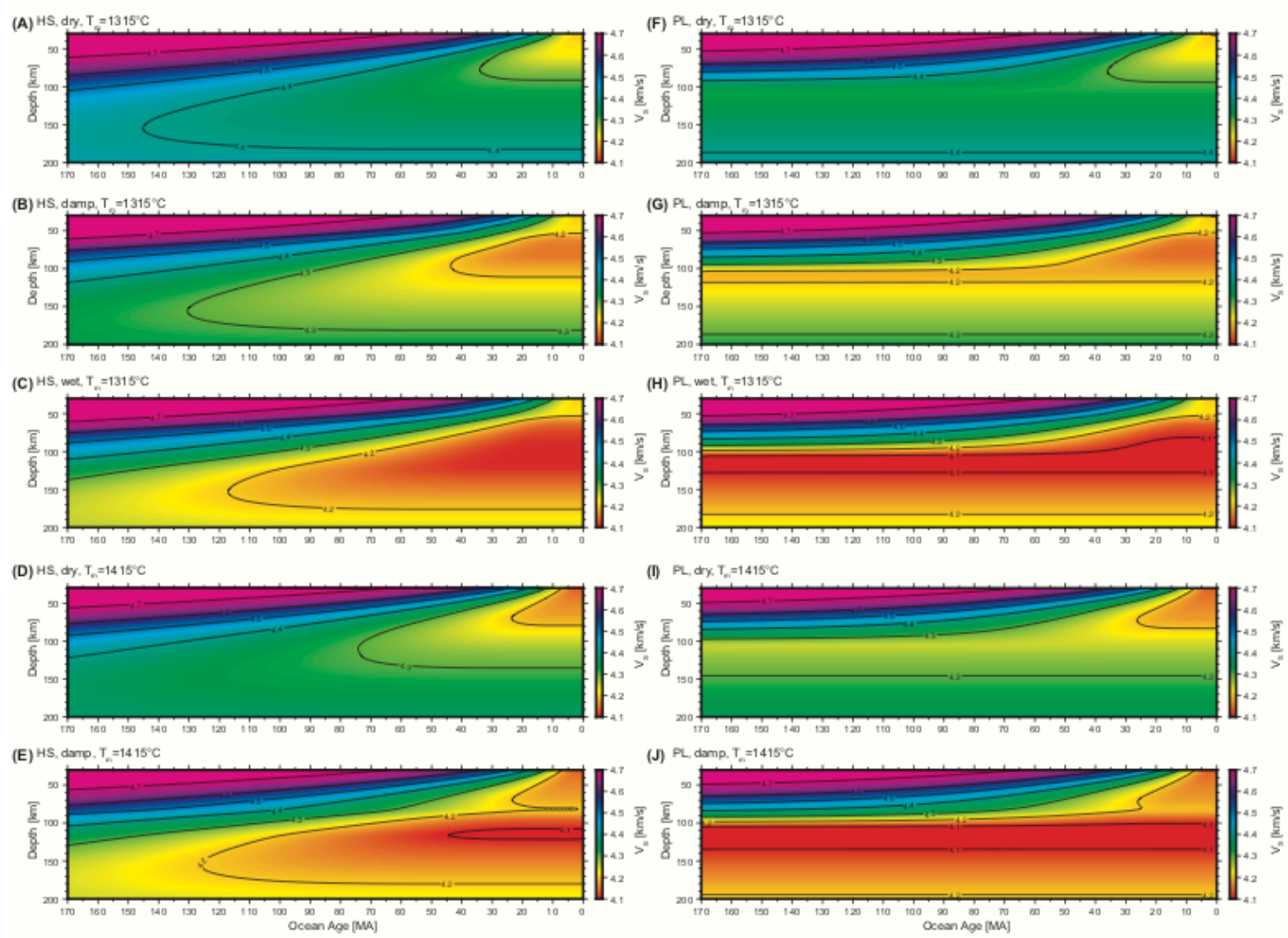

Figure S5: Synthetic seismic velocities for a halfspace (left) and a plate cooling (right) model using attenuation model $\mathrm{Q}_{\mathrm{g}}$. a combined experimental and empirical model as described in Goes et al. (2012). Thermal parameters were taken from McKenzie et al. (2005) for mantle potential temperatures of $1315^{\circ} \mathrm{C}((\mathrm{A})-(\mathrm{C})$ and $(\mathrm{F})-(\mathrm{H}))$ and $1415^{\circ} \mathrm{C}$ ((D)-(E) and (I)-(J)). In all cases, velocities were determined for a dry mantle ((A), (D), $(\mathrm{F})$, and $(\mathrm{I}))$ and a "damp" mantle $\left(1000 \mathrm{H} / 10^{6} \mathrm{Si}\right)((\mathrm{B}),(\mathrm{G}),(\mathrm{E})$, and $(\mathrm{J}))$. We also tested a wetter mantle $\left(3000 \mathrm{H} / 10^{6} \mathrm{Si}\right)$ for the lower temperature case $((\mathrm{C})$ and $(\mathrm{H}))$. 
$\mathrm{HSC}, \mathrm{dry}, \mathrm{T}_{\mathrm{m}}=1325^{\circ} \mathrm{C}$
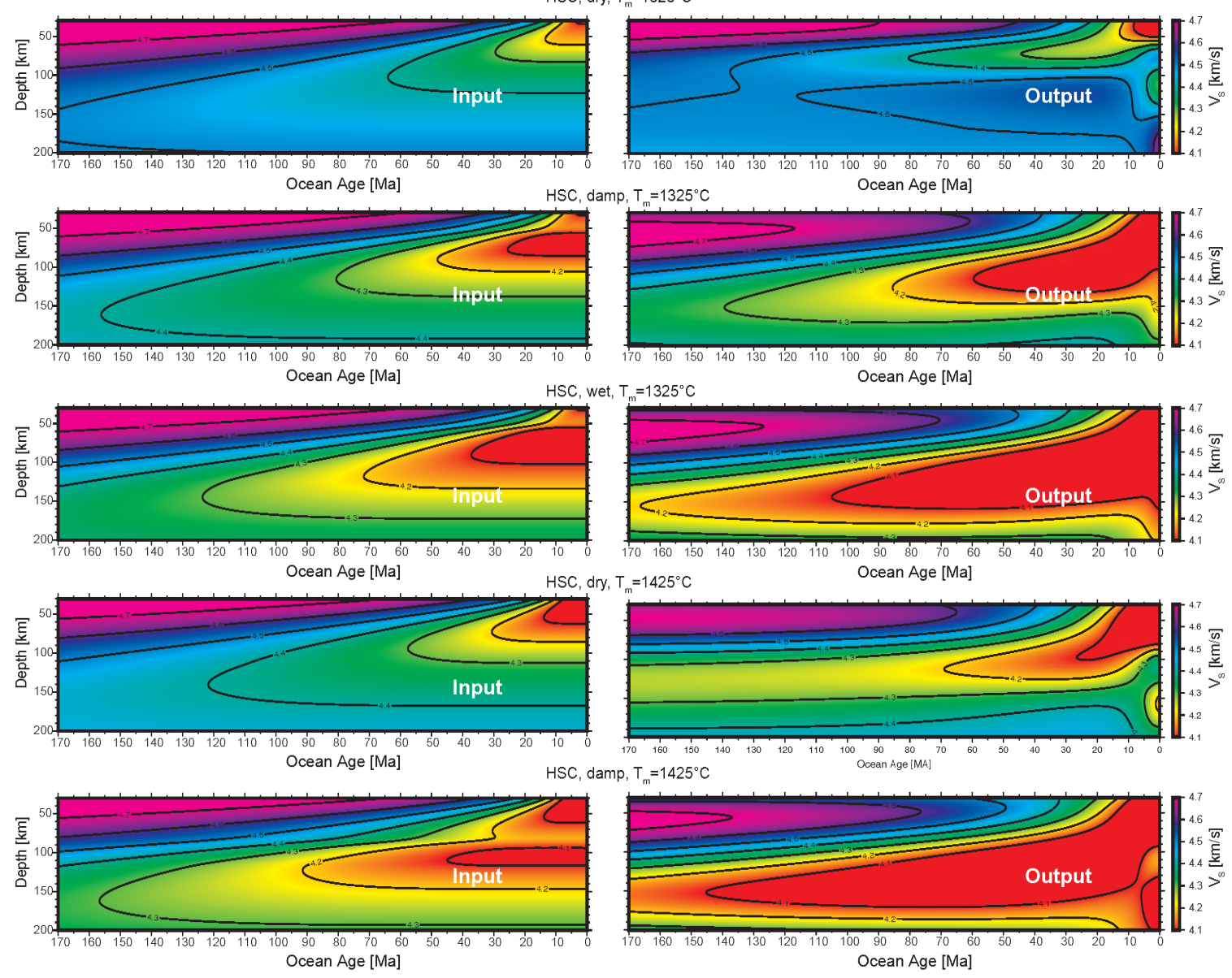

Figure S6: Synthetic tests using the synthetic HSC V models of Fig. 14 as input with dataset 2 . 
$\mathrm{PL}$, dry, $\mathrm{T}_{\mathrm{m}}=1325^{\circ} \mathrm{C}$
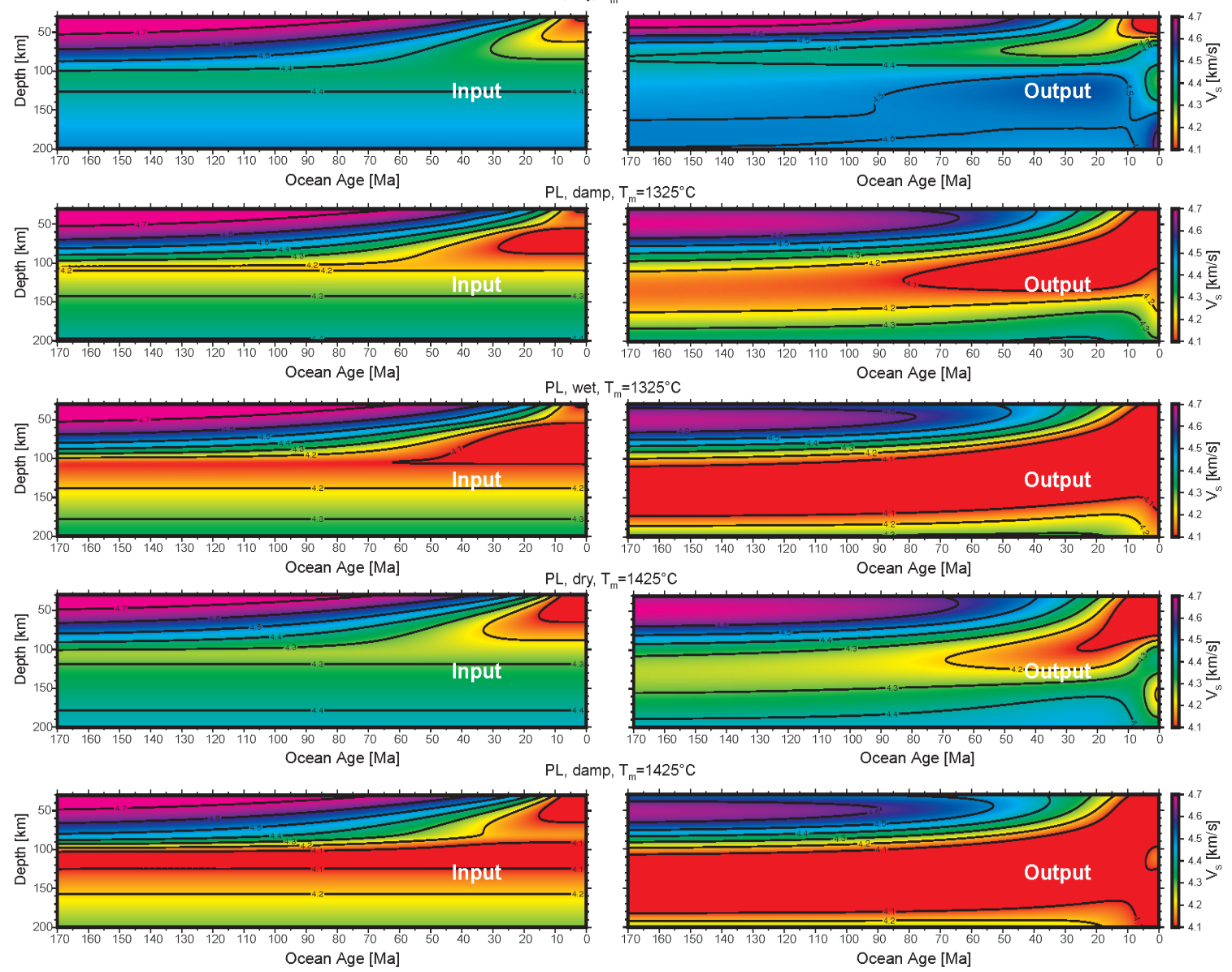

Figure S7: Synthetic tests using the synthetic plate $V_{S}$ models of Fig. 14 as input with dataset 2 . 
HSC, dry, $T_{m}=1325^{\circ} \mathrm{C}$
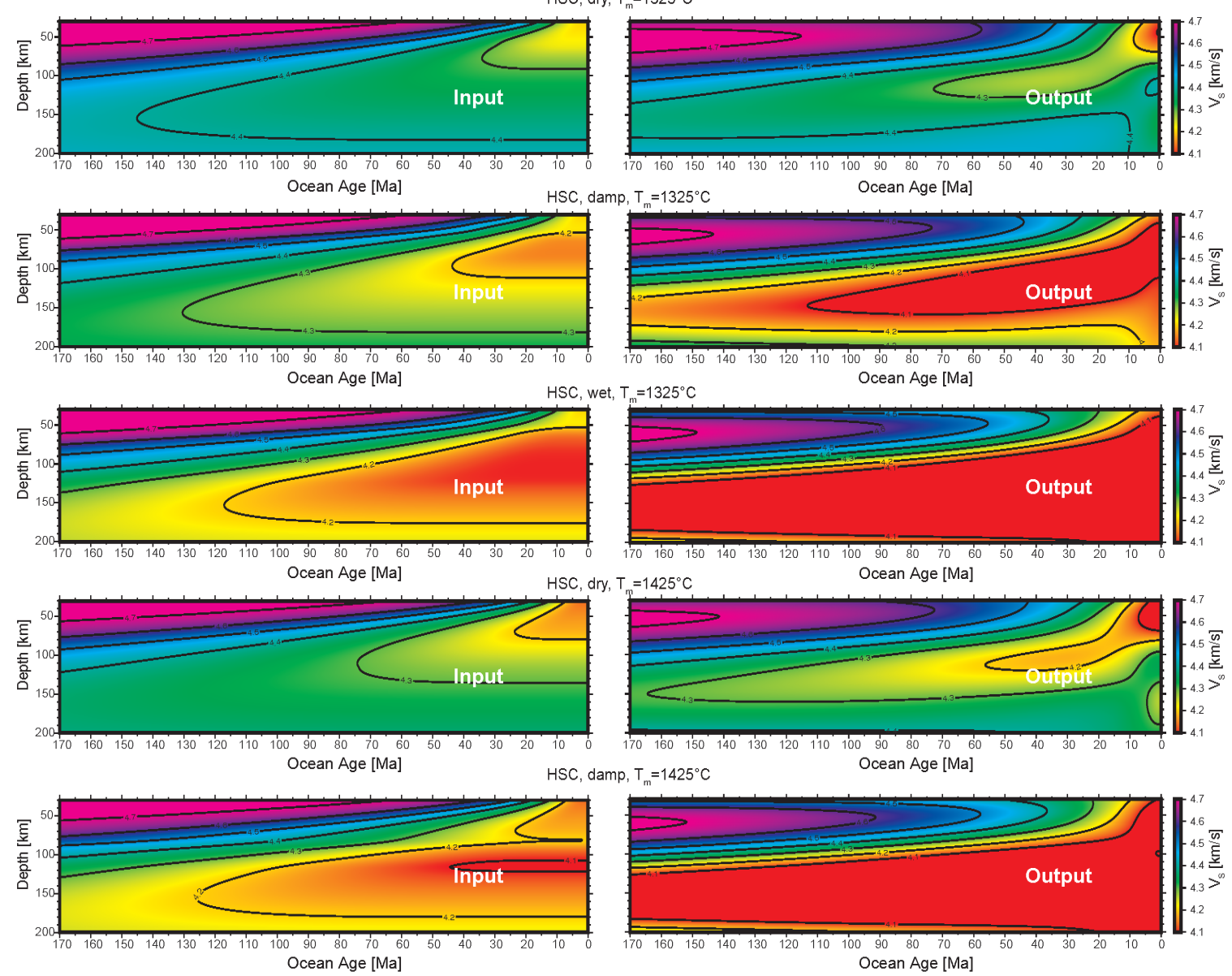

Figure S8: Synthetic tests using the synthetic HSC V $\mathrm{V}_{\mathrm{S}}$ models of Fig. S5 as input with dataset 2 . 
$\mathrm{PL}, \mathrm{dry}, \mathrm{T}_{\mathrm{m}}=1325^{\circ} \mathrm{C}$
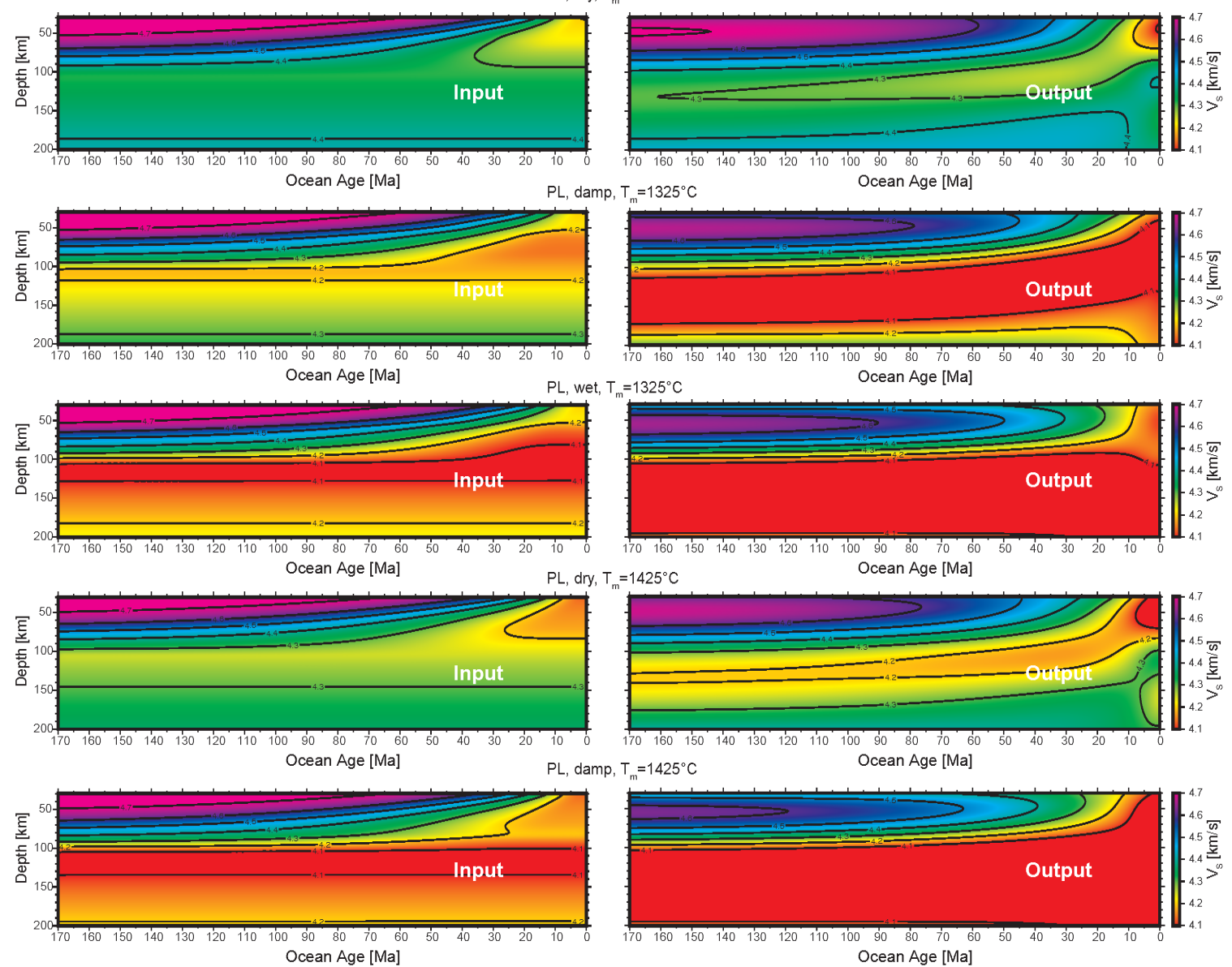

Figure 9: Synthetic tests using the synthetic plate $V_{S}$ models of Fig. S5 as input with dataset 2 . 

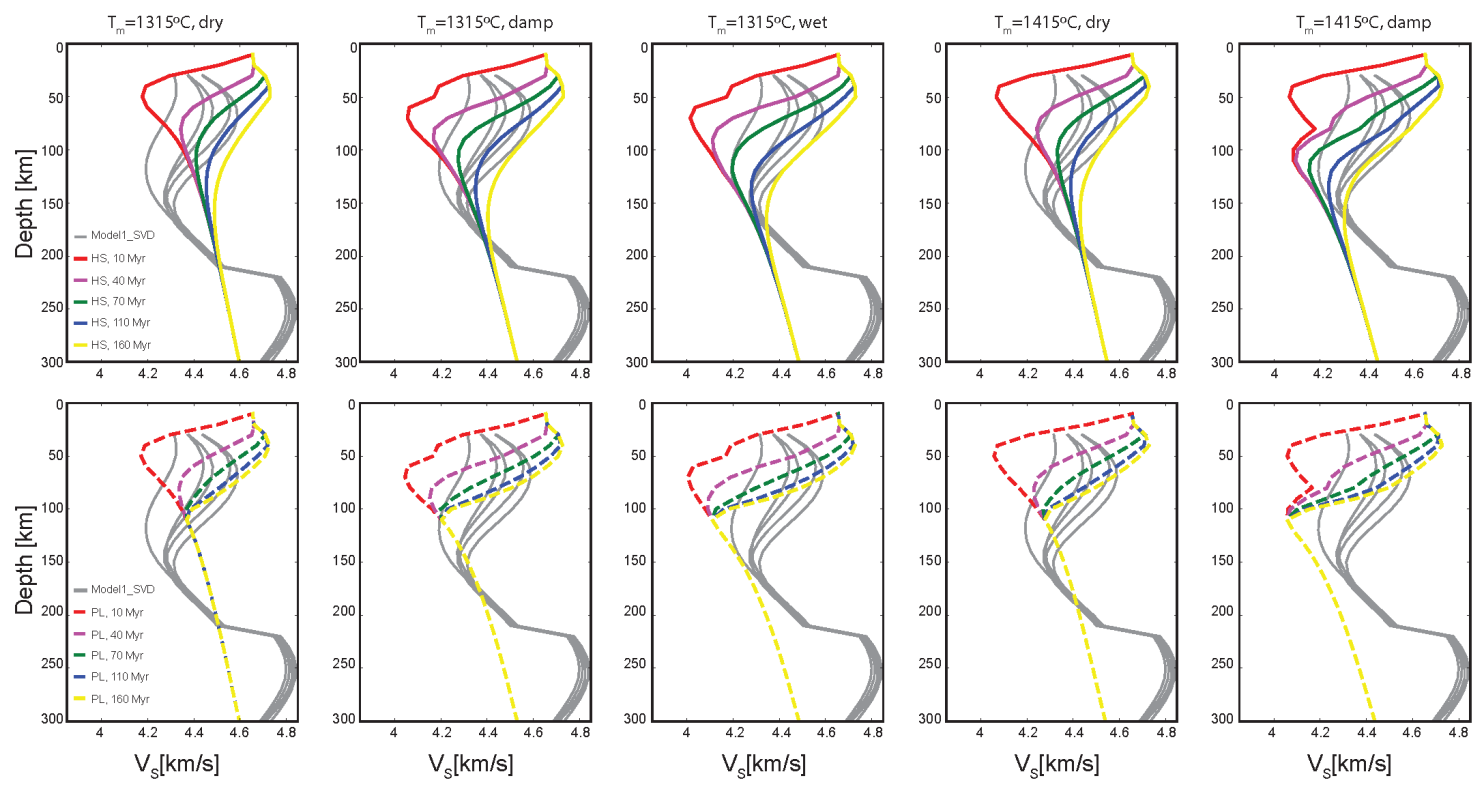

Figure S10: Synthetic $\mathrm{V}_{\mathrm{S}}$ depth profiles for attenuation model $\mathrm{Q}_{\mathrm{g}}$ at different crustal ages and velocity profiles resulting from regularized inversion of dataset 1 for the same ages.
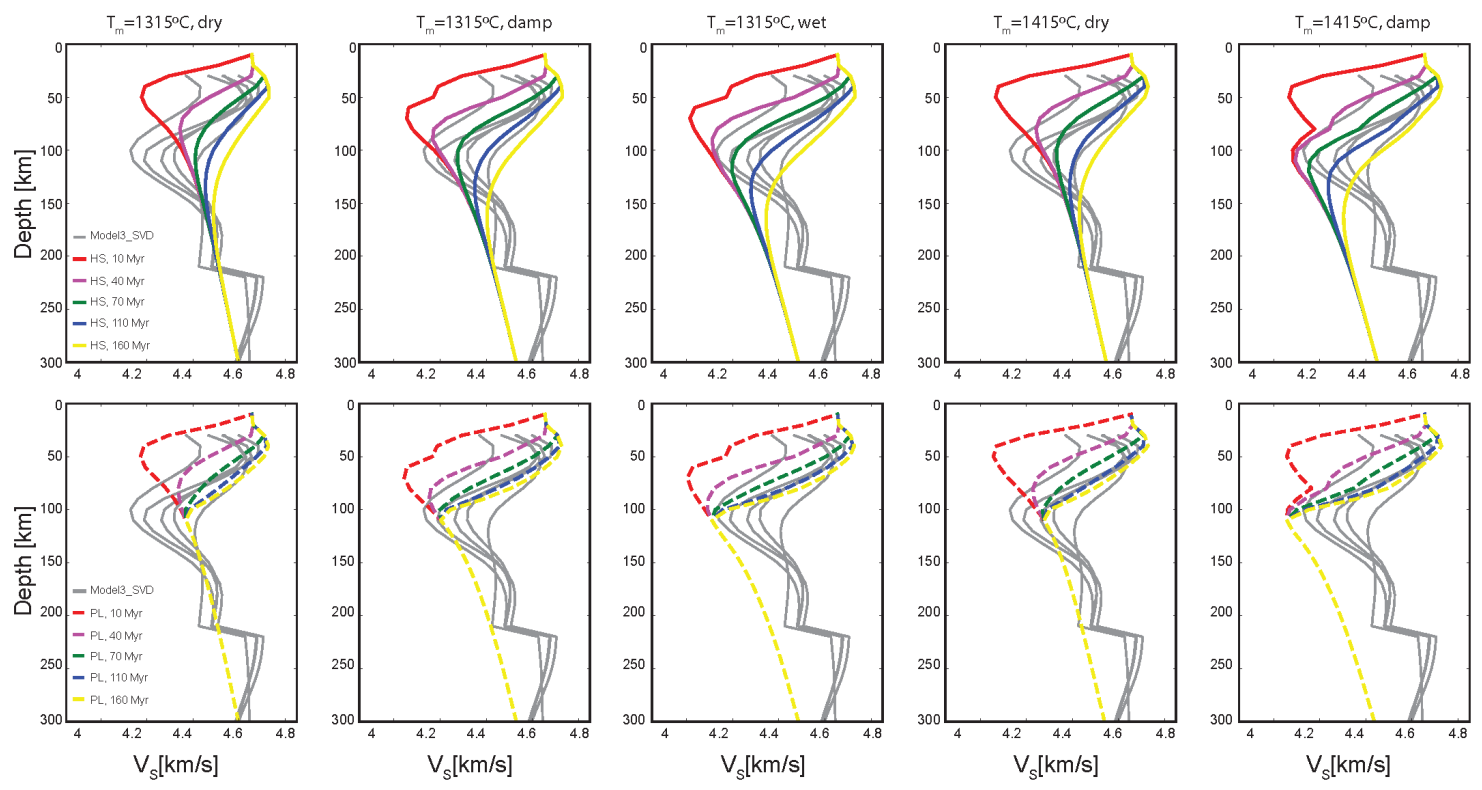

Figure 11: Synthetic $V_{S}$ depth profiles for attenuation model $Q_{g}$ at different crustal ages and velocity profiles resulting from regularized inversion of dataset 3 for the same ages. 

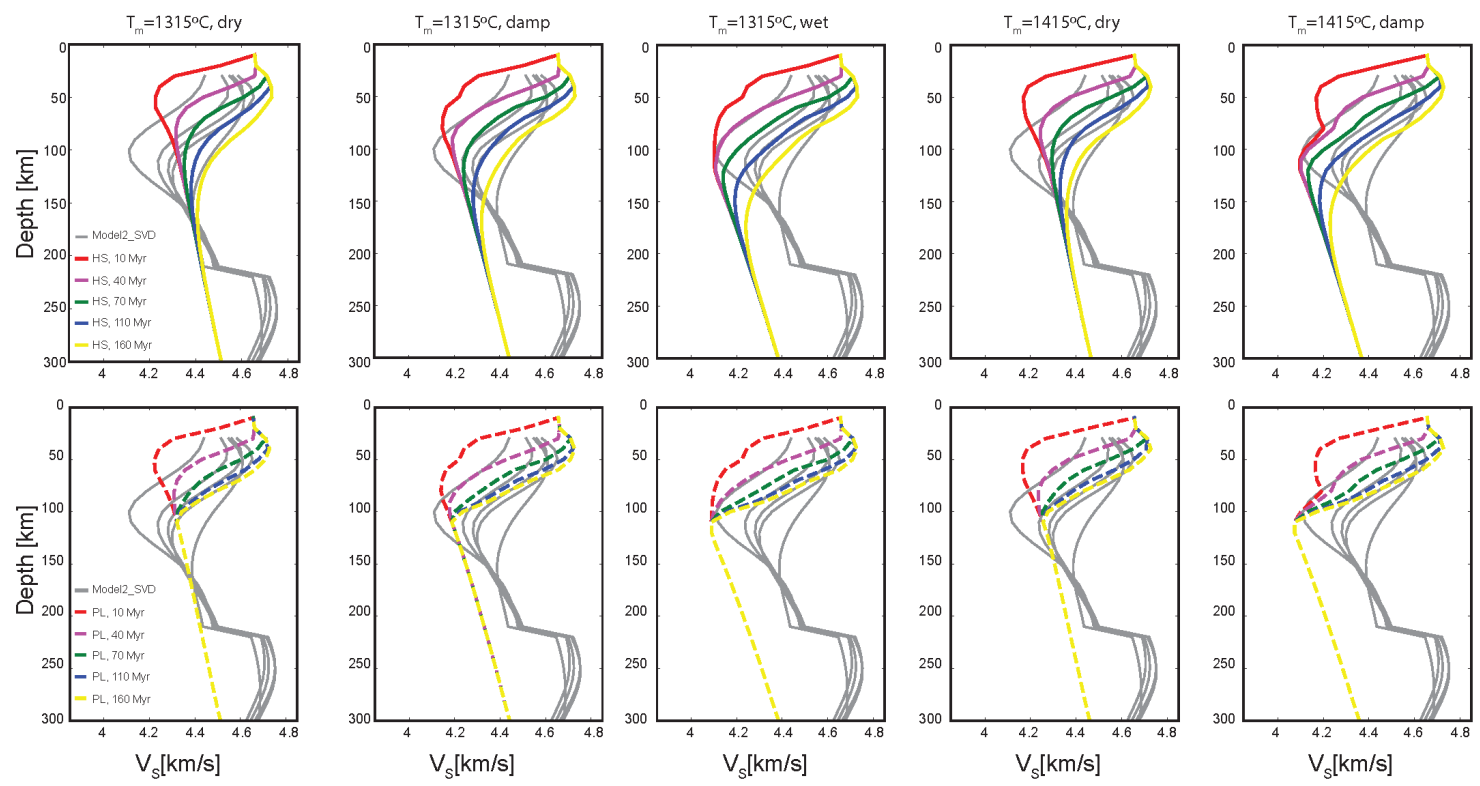

Figure 12: Synthetic $V_{S}$ depth profiles for attenuation model $Q_{J}$ at different crustal ages and velocity profiles resulting from regularized inversion of dataset 2 for the same ages.
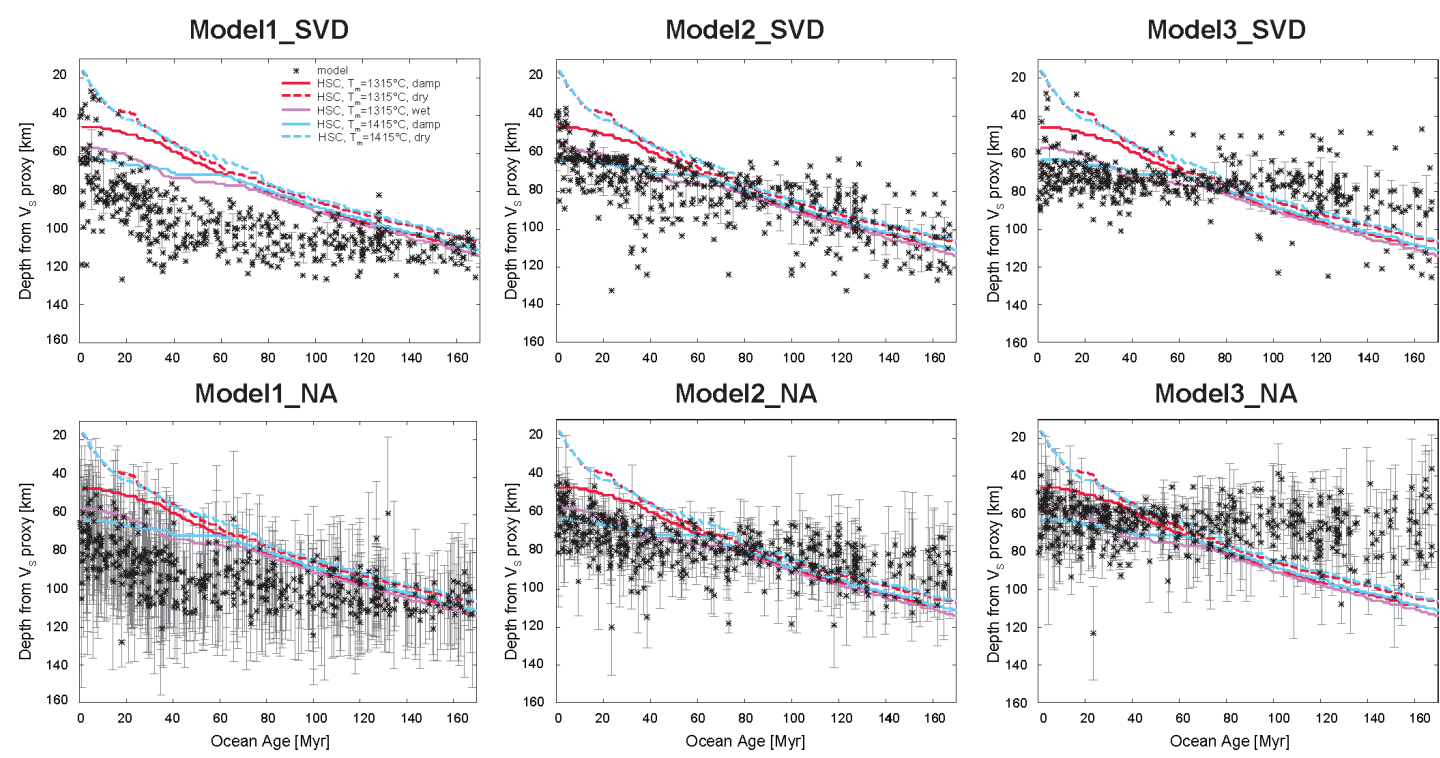

Figure S13: $\mathrm{V}_{\mathrm{S}}$-defined LAB depth proxy determined from our tomographic models and from the synthetic velocity models of Goes et al. (2012) calculated for HSC and attenuation model $\mathrm{Q}_{\mathrm{J}}$. 